\title{
Backward SLE and the symmetry of the welding
}

\author{
Steffen Rohde*1 and Dapeng Zhan ${ }^{\dagger 2}$ \\ ${ }^{1}$ University of Washington \\ ${ }^{2}$ Michigan State University
}

August 8, 2018

\begin{abstract}
The backward chordal Schramm-Loewner Evolution naturally defines a conformal welding homeomorphism of the real line. We show that this homeomorphism is invariant under the automorphism $x \mapsto-1 / x$, and conclude that the associated solution to the welding problem (which is a natural renormalized limit of the finite time Loewner traces) is reversible. The proofs rely on an analysis of the action of analytic circle diffeomorphisms on the space of hulls, and on the coupling techniques of the second author.
\end{abstract}

\section{Contents}

1 Introduction 2

1.1 Introduction and results $\ldots \ldots \ldots \ldots \ldots \ldots \ldots$

1.2 Notation . . . . . . . . . . . . . . . . . . . . . 3

2 Extension of Conformal Maps 4

2.1 Interior hulls in $\mathbb{C} \ldots \ldots \ldots \ldots \ldots \ldots$

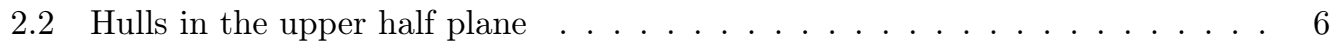

2.3 Hulls in the unit disc . . . . . . . . . . . . . . . . . 14

3 Loewner Equations and Loewner Chains 17

3.1 Forward Loewner equations . . . . . . . . . . . . . . . . . . . 177

3.2 Backward Loewner equations . . . . . . . . . . . . . . . . . . 18

3.3 Normalized global backward trace . . . . . . . . . . . . . . . . . . 20 20

3.4 Forward and backward Loewner chains . . . . . . . . . . . . . . . . . . 21

3.5 Simple curves and weldings . . . . . . . . . . . . . . . . 23

${ }^{*}$ Research partially supported by NSF grant DMS-1068105.

${ }^{\dagger}$ Research partially supported by NSF grants DMS-0963733, DMS-1056840, and Sloan fellowship. 
4 Conformal Transformations 24

4.1 Transformations between backward $\mathbb{H}-$-Loewner chains . . . . . . . . . . . . . 25

4.2 Transformations involving backward $\mathbb{D}$-Loewner chains . . . . . . . . . . . 26

4.3 Conformal invariance of backward $\operatorname{SLE}(\kappa ; \rho)$ processes . . . . . . . . . 27

5 Commutation Relations 30

5.1 Ensemble . . . . . . . . . . . . . . . . . . . . . 30

5.2 Coupling measures . . . . . . . . . . . . . . . . . . . . . . 34

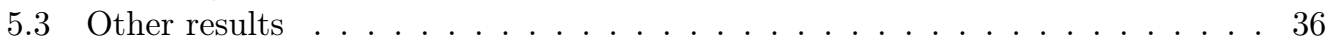

6 Reversibility of Backward Chordal SLE 37

Appendices 40

A Carathéodory Topology 40

B Topology on Interior Hulls 40

C Topology on $\mathbb{H}$-hulls

D Topology on $\mathbb{D}$-hulls 42

\section{Introduction}

\subsection{Introduction and results}

The Schramm-Loewner Evolution $\mathrm{SLE}_{\kappa}$, first introduced in [14], is a stochastic process of random conformal maps that has received a lot of attention over the last decade. We refer to the introductory text [6] for basic facts and definitions. In this paper we are largely concerned with chordal $\mathrm{SLE}_{\kappa}$, which can be viewed as a family of random curves $\gamma$ that join 0 and $\infty$ in the closure of the upper half plane $\overline{\mathbb{H}}$. A fundamental property of chordal SLE is reversibility: The law of $\gamma$ is invariant under the automorphism $z \mapsto-1 / z$ of $\mathbb{H}$, modulo time parametrization. This has first been proved by the second author in 18 , for $\kappa \leq 4$, and recently by Miller and Sheffield for $4<\kappa \leq 8$ in [9]. It is known to be false for $\kappa>8$ $([13,[19])$.

In the early years of SLE, Oded Schramm, Wendelin Werner and the first author made an attempt to prove reversibility along the following lines: The "backward" flow

$$
\partial_{t} f_{t}(z)=\frac{-2}{f_{t}(z)-\sqrt{\kappa} B_{t}}, \quad f_{0}(z)=z, \quad 0 \leq t \leq T,
$$

generates curves $\beta_{T}=\beta[0, T]$ whose law is that of the chordal SLE trace $\gamma[0, T]$ (up to translation by $\sqrt{\kappa} B_{T}$ ). When $\kappa \leq 4$, these curves are simple, and each point of $\beta$ (with the exception of the endpoints) corresponds to two points on the real line under the conformal map $f_{t}$. The conformal welding homeomorphism $\phi$ of $\beta_{T}$ is the auto-homeomorphism of the interval $f_{T}^{-1}\left(\beta_{T}\right)$ that interchanges these two points. In other words, it is the rule that describes which points on the real line are to be identified (laminated) in order to form the curve $\beta_{T}$. It is known [13] that, for $\kappa<4$, the welding almost surely uniquely determines the curve. The welding homeomorphism can be obtained by restricting the backward flow 
to the real line: Two points $x \neq y$ on the real line are to be welded if and only if their swallowing times coincide, $\phi(x)=y$ if and only if $\tau_{x}=\tau_{y}$, see Section 3.5 An idea to prove reversibility was to prove the invariance of $\phi$ under $x \mapsto-1 / x$, and to relate this to reversibility of a suitable limit of the curves $\beta_{T}$. But the attempts to prove invariance of $\phi$ failed, and this program was never completed successfully.

In this paper, we use the coupling techniques of the second author, introduced in [18 for his proof of reversibility of (forward) SLE traces. We use it to prove the invariance of the welding:

Theorem 1.1. Let $\kappa \in(0,4]$, and $\phi$ be a backward chordal $S L E_{\kappa}$ welding. Let $h(z)=-1 / z$. Then $h \circ \phi \circ h$ has the same distribution as $\phi$.

As a consequence, in the range $\kappa \in(0,4)$ where the SLE trace is conformally removable, we obtain the reversibility of suitably normalized limits of the $\beta_{T}$ (see Section 6 for details):

Theorem 1.2. Let $\kappa \in(0,4)$, and $\beta$ be a normalized global backward chordal $S L E_{\kappa}$ trace. Let $h(z)=-1 / z$. Then $h(\beta \backslash\{0\})$ has the same distribution as $\beta \backslash\{0\}$ as random sets.

In the important paper [16], Sheffield obtains a representation of the SLE welding in terms of a quantum gravity boundary length measure, and also relates it to a simple Jordan arc, which differs from our $\beta$ only through normalization. A similar random welding homeomorphism is constructed in [2, where the main point is the very difficult existence of a curve solving the welding problem. Our approach to the welding is different: In order to prove Theorem 1.1, in Section 2 we develop a framework to study the effect of analytic perturbations of weldings on the corresponding hulls. We show in Section 4 that a Möbius image of a backward chordal $\mathrm{SLE}_{\kappa}$ process is a backward radial $\operatorname{SLE}(\kappa,-\kappa-6)$ process, and the welding is preserved under this conformal transformation. In Section 5 we apply the coupling technique to show that backward radial $\operatorname{SLE}(\kappa,-\kappa-6)$ started from an ordered pair of points $(a, b)$ commutes with backward radial SLE $(\kappa,-\kappa-6)$ started from $(b, a)$, and use this in Section 6 to prove Theorem 1.1

In a subsequent paper [23] of the second author, Theorem 1.1 is used to study the ergodic properties of a forward $\mathrm{SLE}_{\kappa}$ trace near the tip at a fixed capacity time.

\subsection{Notation}

Let $\widehat{\mathbb{C}}=\mathbb{C} \cup\{\infty\}, \mathbb{D}=\{z \in \mathbb{C}:|z|<1\}, \mathbb{D}^{*}=\widehat{\mathbb{C}} \backslash \overline{\mathbb{D}}, \mathbb{T}=\{z \in \mathbb{C}:|z|=1\}$, and $\mathbb{H}=\{z \in \mathbb{C}: \operatorname{Im} z>0\}$. Let $I_{\mathbb{R}}(z)=\bar{z}$ and $I_{\mathbb{T}}(z)=1 / \bar{z}$ be the reflections about $\mathbb{R}$ and $\mathbb{T}$, respectively. Let $e^{i}$ denote the map $z \mapsto e^{i z}$. Let $\cot _{2}(z)=\cot (z / 2)$ and $\sin _{2}(z)=\sin (z / 2)$. For a real interval $J$, let $C(J)$ denote the space of real valued continuous functions on $J$. An increasing or decreasing function in this paper is assumed to be strictly monotonic. We use $B(t)$ to denote a standard real Brownian motion. By $f: D \stackrel{\text { Conf }}{\rightarrow} E$ we mean that $f$ maps $D$

conformally onto $E$. By $f_{n} \stackrel{\text { l.u. }}{\longrightarrow} f$ in $U$ we mean that $f_{n}$ converges to $f$ uniformly on every compact subset of $U$. We will frequently use the notation $D_{n} \stackrel{\text { Cara }}{\longrightarrow} D$ as in Definition A.1.

The outline of this paper is the following. In Section 2, we derive some fundamental results in Complex Analysis, which are interesting on their own. In Section 3, we review the properties of forward Loewner processes, and derive some properties of backward Loewner processes. In Section 4, we discuss how are backward Loewner processes transformed by conformal maps. In Section 5 we present and prove certain commutation relations between backward $\operatorname{SLE}(\kappa ; \vec{\rho})$ processes. In the last section, we prove the reversibility of backward 
chordal $\mathrm{SLE}_{\kappa}$ processes for $\kappa \in(0,4]$ and propose questions in other cases. In the appendix, we discuss some results on the topology of domains and hulls.

\section{Extension of Conformal Maps}

\subsection{Interior hulls in $\mathbb{C}$}

An interior hull (in $\mathbb{C}$ ) is a nonempty compact connected set $K \subset \mathbb{C}$ such that $\mathbb{C} \backslash K$ is also connected. For every interior hull $K$ in $\mathbb{C}$, there are a unique $r \geq 0$ and a unique $\phi_{K}: \widehat{\mathbb{C}} \backslash K \stackrel{\text { Conf }}{\rightarrow} \widehat{\mathbb{C}} \backslash r \overline{\mathbb{D}}$ such that $\phi_{K}(\infty)=\infty$ and $\phi_{K}^{\prime}(\infty):=\lim _{z \rightarrow \infty} z / \phi_{K}(z)=1$. We call $\operatorname{rad}(K):=r$ the radius of $K$ and $\operatorname{cap}(K):=\ln (r)$ the capacity of $K$. The radius is 0 iff $K$ contains only one point. In general, we have $\operatorname{rad}(K) \leq \operatorname{diam}(K) \leq 4 \operatorname{rad}(K)$. We call $K$ nondegenerate if it contains more than one point. For such $K$, there is a unique $\varphi_{K}: \widehat{\mathbb{C}} \backslash K \stackrel{\text { Conf }}{\rightarrow} \mathbb{D}^{*}$ such that $\varphi_{K}(\infty)=\infty$ and $\varphi_{K}^{\prime}(\infty)>0$. In fact, $\varphi_{K}=\phi_{K} / \operatorname{rad}(K)$. Let $\psi_{K}=\varphi_{K}^{-1}$ for such $K$.

For any Jordan curve $J$ in $\mathbb{C}$, let $D_{J}$ denote the Jordan domain bounded by $J$, and let $D_{J}^{*}=\widehat{\mathbb{C}} \backslash\left(D_{J} \cup J\right)$. Suppose $f_{J}: \mathbb{D} \stackrel{\text { Conf }}{\rightarrow} D_{J}$ and $f_{J}^{*}=\psi_{\overline{D_{J}}}: \mathbb{D}^{*} \stackrel{\text { Conf }}{\rightarrow} D_{J}^{*}$. Then both $f_{J}$ and $f_{J}^{*}$ extend continuously to a homeomorphism from $\mathbb{T}$ onto $J$. Let $h=\left(f_{J}^{*}\right)^{-1} \circ f_{J}$. Then $h$ is an orientation-preserving automorphism of $\mathbb{T}$. We call such $h$ a conformal welding. Not every homeomorphism of $\mathbb{T}$ is a conformal welding, but it is well-known (and an easy consequence of the uniformization theorem) that every analytic automorphism is a conformal welding, and that the associated Jordan curve is analytic. See 8 for the quasiconformal theory of conformal welding, and [3] for deep generalizations and further references.

Lemma 2.1. Let $\beta$ be an analytic Jordan curve. Let $\Omega \subset \mathbb{C}$ be a neighborhood of $\mathbb{T}$. Suppose $W$ is a conformal map defined in $\Omega$, maps $\mathbb{T}$ onto $\mathbb{T}$, and preserves the orientation of $\mathbb{T}$. Let $\Omega^{\beta}=\beta \cup D_{\beta} \cup \psi_{\overline{D_{\beta}}}\left(\Omega \cap \mathbb{D}^{*}\right)$. Then there is a conformal map $V$ defined in $\Omega^{\beta}$ such that $V \circ \psi_{\overline{D_{\beta}}}=\psi_{\overline{D_{V(\beta)}}} \circ W$ in $\Omega \cap \mathbb{D}^{*}$.

Proof. Fix a conformal map $f_{\beta}: \mathbb{D} \stackrel{\text { Conf }}{\rightarrow} D_{\beta}$ and let $h_{\beta}=\varphi_{\overline{D_{\beta}}} \circ f_{\beta}$ be the associated conformal welding homeomorphism. Define $h=W \circ h_{\beta}$. Since $\beta$ is analytic, $h$ is analytic and there is an analytic Jordan curve $\gamma$ and a conformal map $f_{\gamma}: \mathbb{D} \stackrel{\text { Conf }}{\rightarrow} D_{\gamma}$ such that $h=h_{\gamma}=\varphi_{\overline{D_{\gamma}}} \circ f_{\gamma}$. Define $V=f_{\gamma} \circ f_{\beta}^{-1}$ on $D_{\beta}$. Since $\beta$ and $\gamma$ are analytic curves, $V$ extends conformally to a neighborhood of $\beta$ with $V(\beta)=\gamma$. On $\beta$, this extension (still denoted $V)$ satisfies $V=\left(\psi_{\overline{D_{\gamma}}} \circ h_{\gamma}\right) \circ\left(h_{\beta}^{-1} \circ \psi_{\overline{D_{\beta}}}^{-1}\right)=\psi_{\overline{D_{\gamma}}} \circ W \circ \psi_{\overline{D_{\beta}}}^{-1}$. Therefore $V$ extends conformally to all of $\Omega^{\beta}$ and satisfies the desired property.

Theorem 2.2. Let $H$ be a nondegenerate interior hull. Let $\Omega \subset \mathbb{C}$ be a neighborhood of $\mathbb{T}$. Suppose $W$ is a conformal map defined in $\Omega$, maps $\mathbb{T}$ onto $\mathbb{T}$, and preserves the orientation of $\mathbb{T}$. Let $\Omega^{H}=H \cup \psi_{H}\left(\Omega \cap \mathbb{D}^{*}\right)$. Then there is a conformal map $V$ defined in $\Omega^{H}$ such that $V \circ \psi_{H}=\psi_{V(H)} \circ W$ in $\Omega \cap \mathbb{D}^{*}$. If another conformal map $\widetilde{V}$ satisfies the properties of $V$, then $\widetilde{V}=a V+b$ for some $a>0$ and $b \in \mathbb{C}$. 


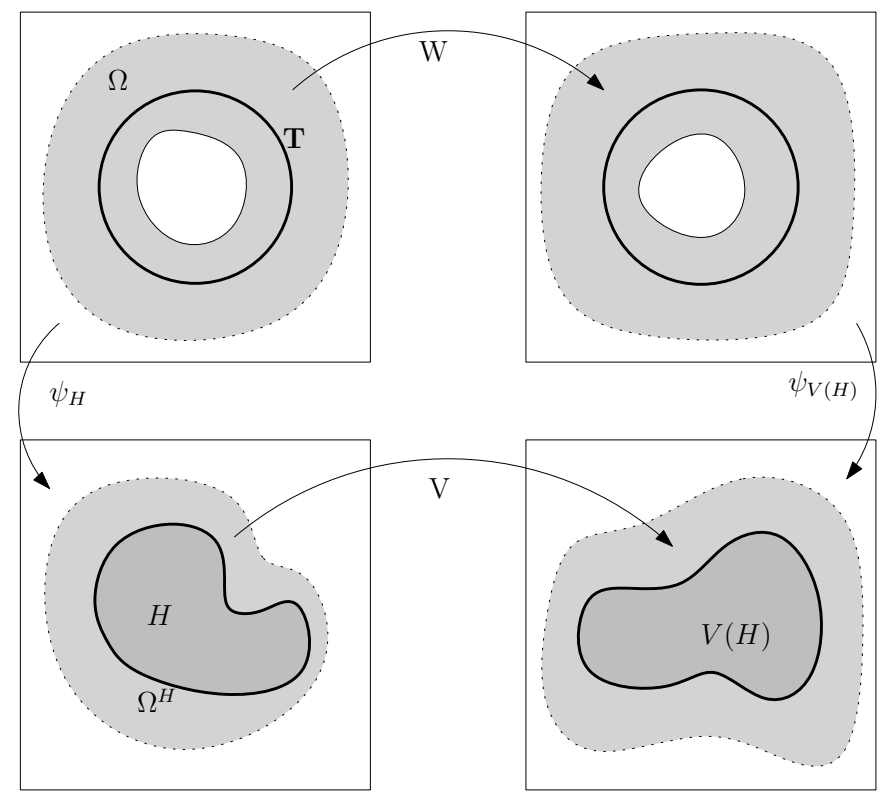

Figure 1: The situation of Theorem 2.2 and Lemma 2.1. Given $H$ and $W, V$ can be constructed to be analytic in $H$. In Lemma 2.1, the boundary of $H$ is assumed to be an analytic Jordan curve, while in Theorem 2.2. no regularity assumption is made.

Proof. First, define a sequence of analytic Jordan curves $\left(\beta_{n}\right)$ by

$$
\beta_{n}=\psi_{H}\left(\left\{e^{\frac{1}{n}+i \theta}: 0 \leq \theta \leq 2 \pi\right\}\right), \quad n \in \mathbb{N} .
$$

Then $\beta_{n} \cup D_{\beta_{n}} \rightarrow H$ in $d_{\mathcal{H}}$ (see Appendix B). From Lemma 2.1, for each $n \in \mathbb{N}$, there is a conformal map $V_{n}$ defined in $\Omega^{\beta_{n}}:=\beta_{n} \cup D_{\beta_{n}} \cup \psi_{\beta_{n}}\left(\Omega \cap \mathbb{D}^{*}\right)$ such that $V_{n} \circ \psi_{\beta_{n}}=\psi_{V_{n}\left(\beta_{n}\right)} \circ W$ in $\Omega \cap \mathbb{D}^{*}$. Note that for any $a_{n}>0$ and $b_{n} \in \mathbb{C}, a_{n} V_{n}+b_{n}$ satisfies the same property as $V_{n}$. Thus, we may assume that $0 \in V_{n}\left(\beta_{n}\right) \subset \overline{\mathbb{D}}$ and $V_{n}\left(\beta_{n}\right) \cap \mathbb{T} \neq \emptyset$. Let $\gamma_{n}=V_{n}\left(\beta_{n}\right)$, $n \in \mathbb{N}$. Then each $\gamma_{n}$ is an interior hull contained in the interior hull $\overline{\mathbb{D}}$, and $\operatorname{diam}\left(\gamma_{n}\right) \geq 1$. So $\operatorname{rad}\left(\gamma_{n}\right) \geq 1 / 4$. From Corollary B.2. $\left(\gamma_{n}\right)$ contains a subsequence which converges to some interior hull $K$ contained in $\overline{\mathbb{D}}$ with radius at least $1 / 4$. So $K$ is nondegenerate. By passing to a subsequence, we may assume that $\gamma_{n} \rightarrow K$. From $\beta_{n} \rightarrow H$ and $\gamma_{n} \rightarrow K$ we get $\psi_{\beta_{n}} \stackrel{\text { l.u. }}{\longrightarrow} \psi_{H}$ in $\Omega \cap \mathbb{D}^{*}$ and $\psi_{\gamma_{n}} \stackrel{\text { l.u. }}{\longrightarrow} \psi_{K}$ in $W\left(\Omega \cap \mathbb{D}^{*}\right)$. Thus, $\psi_{\beta_{n}}\left(\Omega \cap \mathbb{D}^{*}\right) \stackrel{\text { Cara }}{\longrightarrow} \psi_{H}\left(\Omega \cap \mathbb{D}^{*}\right)$ by Lemma A.2.

Since $V_{n} \circ \psi_{\beta_{n}}=\psi_{\gamma_{n}} \circ W$ in $\Omega \cap \mathbb{D}^{*}$, we find that $V_{n}=\psi_{\gamma_{n}} \circ W \circ \psi_{\beta_{n}}^{-1}$ in $\psi_{\beta_{n}}\left(\Omega \cap \mathbb{D}^{*}\right)$. Let $V=\psi_{K} \circ W \circ \psi_{H}^{-1}$ in $\psi_{H}\left(\Omega \cap \mathbb{D}^{*}\right)$. Then $V_{n} \stackrel{\text { l.u. }}{\longrightarrow} V$ in $\psi_{H}\left(\Omega \cap \mathbb{D}^{*}\right)$. We may find $r>1$ such that for any $s \in(1, r], s \mathbb{T} \subset \Omega \cap \mathbb{D}^{*}$. Then $\psi_{H}(r \mathbb{T})$ is a Jordan curve in $\psi_{H}\left(\Omega \cap \mathbb{D}^{*}\right)$ surrounding $H$, and the Jordan domain bounded by $\psi_{H}(r \mathbb{T})$ is contained in $\Omega^{H}=H \cup \psi_{H}\left(\Omega \cap \mathbb{D}^{*}\right)$. Since $\psi_{H}(r \mathbb{T})$ is a compact subset of $\psi_{H}\left(\Omega \cap \mathbb{D}^{*}\right)$, we have $V_{n} \rightarrow V$ uniformly on $\psi_{H}(r \mathbb{T})$. It is easy to see that $\Omega^{\beta_{n}} \stackrel{\text { Cara }}{\longrightarrow} \Omega^{H}$. For $n$ big enough, $\psi_{H}(r \mathbb{T})$ together with its interior is contained in $\Omega^{\beta_{n}}$. From the maximum principle, $V_{n}$ converges uniformly in the interior of $\psi_{H}(r \mathbb{T})$ to a conformal map which extends $V$. We still use $V$ to denote 
the extended conformal map. Then $V$ is a conformal map defined in $\Omega^{H}$, and $V_{n} \stackrel{\text { l.u. }}{\longrightarrow} V$ in $\Omega^{H}$. Letting $n \rightarrow \infty$ in the equality $V_{n} \circ \psi_{\beta_{n}}=\psi_{\gamma_{n}} \circ W$ in $\Omega \cap \mathbb{D}^{*}$, we conclude that $V \circ \psi_{H}=\psi_{V(H)} \circ W$ in $\Omega \cap \mathbb{D}^{*}$. So the existence part is proved.

If $\widetilde{V}=a V+b$ for some $a>0$ and $b \in \mathbb{C}$, then $\psi_{\widetilde{V}(H)}=a \psi_{V(H)}+b$, which implies $\widetilde{V} \circ \psi_{H}=\psi_{\widetilde{V}(H)} \circ W$. Finally, suppose $\widetilde{V}$ satisfies the properties of $V$. Then $\widetilde{V} \circ V^{-1}$ is a conformal map in $V\left(\Omega^{H}\right)$. Since $V \circ \psi_{H}=\psi_{V(H)} \circ W$ and $\widetilde{V} \circ \psi_{H}=\psi_{\widetilde{V}(H)} \circ W$ in $\Omega \cap \mathbb{D}^{*}$, we find that $\widetilde{V} \circ V^{-1}=\psi_{\widetilde{V}(H)} \circ \psi_{V(H)}^{-1}$ in $\psi_{V(H)}\left(W\left(\Omega \cap \mathbb{D}^{*}\right)\right)=V\left(\Omega^{H}\right) \backslash V(H)$. Note that $\psi_{\widetilde{V}(H)} \circ \psi_{V(H)}^{-1}$ is a conformal map defined in $\widehat{\mathbb{C}} \backslash V(H)$. Since $V\left(\Omega^{H}\right) \cup(\widehat{\mathbb{C}} \backslash V(H))=\widehat{\mathbb{C}}$, we may define an analytic function $h$ in $\mathbb{C}$ such that $h=\widetilde{V} \circ V^{-1}$ in $V\left(\Omega^{H}\right)$ and $h=\psi_{\widetilde{V}(H)} \circ \psi_{V(H)}^{-1}$ in $\mathbb{C} \backslash V(H)$. From the properties of $\psi_{\widetilde{V}(H)}$ and $\psi_{V(H)}$, we have $h(\infty)=\infty$ and $h^{\prime}(\infty)>0$. Thus, $h(z)=a z+b$ for some $a>0$ and $b \in \mathbb{C}$, which implies that $\widetilde{V}=a V+b$.

Now we obtain a new proof of the following well-known result about conformal welding.

Corollary 2.3. Let $W$ be conformal in a neighborhood of $\mathbb{T}$, maps $\mathbb{T}$ onto $\mathbb{T}$, and preserves the orientation of $\mathbb{T}$. If $h$ is a conformal welding, then $W \circ h$ and $h \circ W$ are also conformal weldings.

Proof. Apply Theorem 2.2 to $H=\overline{D_{J}}$, where $J$ is the Jordan curve for the conformal welding $h$. We find a conformal map $V$ defined in $\Omega^{H}=D_{J} \cup f_{J}^{*}\left(\Omega \cap \mathbb{D}^{*}\right)$ such that $V \circ f_{J}^{*}=\psi_{V(H)} \circ W$ in $\Omega \cap \mathbb{D}^{*}$. Let $J^{\prime}=V(J)$. Then $J^{\prime}$ is also a Jordan curve, $V(H)=\overline{D_{J^{\prime}}}$, and $\psi_{V(H)}=f_{J^{\prime}}^{*}$. Let $f_{J^{\prime}}=V \circ f_{J}$. Then $f_{J^{\prime}}: \mathbb{D} \stackrel{\text { Conf }}{\rightarrow} D_{J}$. Thus,

$$
W \circ h=W \circ\left(f_{J}^{*}\right)^{-1} \circ f_{J}=\psi_{H(H)}^{-1} \circ V \circ f_{J}=\left(f_{J^{\prime}}^{*}\right)^{-1} \circ f_{J^{\prime}},
$$

which implies that $W \circ h$ is a conformal welding. As for $h \circ W$, note that $(h \circ W)^{-1}=$ $W^{-1} \circ h^{-1}$ and that $h$ is a conformal welding if and only if $h^{-1}$ is a conformal welding.

\subsection{Hulls in the upper half plane}

Let $\mathbb{H}=\{z \in \mathbb{C}: \operatorname{Im} z>0\}$. A subset $K$ of $\mathbb{H}$ is called an $\mathbb{H}$-hull if it is bounded, relatively closed in $\mathbb{H}$, and $\mathbb{H} \backslash K$ is simply connected. For every $\mathbb{H}$-hull $K$, there is are a unique $c \geq 0$ and a unique $g_{K}: \mathbb{H} \backslash K \stackrel{\text { Conf }}{\rightarrow} \mathbb{H}$ such that $g_{K}(z)=z+\frac{c}{z}+O\left(\frac{1}{z^{2}}\right)$ as $z \rightarrow \infty$. The number $c$ is called the $\mathbb{H}$-capacity of $K$, and is denoted by hcap $(K)$. Let $f_{K}=g_{K}^{-1}$. The empty set is an $\mathbb{H}$-hull with hcap $(\emptyset)=0$ and $g_{\emptyset}=f_{\emptyset}=\operatorname{id}_{\mathbb{H}}$.

Definition 2.4. Let $K_{1}$ and $K_{2}$ be $\mathbb{H}$-hulls.

1. If $K_{1} \subset K_{2}$, define $K_{2} / K_{1}=g_{K_{1}}\left(K_{2} \backslash K_{1}\right)$. We call $K_{2} / K_{1}$ a quotient hull of $K_{2}$, and write $K_{2} / K_{1} \prec K_{2}$.

2. The product $K_{1} \cdot K_{2}$ is defined to be $K_{1} \cup f_{K_{1}}\left(K_{2}\right)$.

The following facts are easy to check.

1. $K_{2} / K_{1}$ and $K_{1} \cdot K_{2}$ in the definition are also $\mathbb{H}$-hulls.

2. For any two $\mathbb{H}$-hulls $K_{1}$ and $K_{2}, K_{1} \subset K_{1} \cdot K_{2}$ and $K_{2}=\left(K_{1} \cdot K_{2}\right) / K_{1} \prec K_{1} \cdot K_{2}$. If $K_{1} \subset K_{2}$, then $K_{1} \cdot\left(K_{2} / K_{1}\right)=K_{2}$. 
3. The space of $\mathbb{H}$-hulls with the product "." is a semigroup with identity element $\emptyset$, and " $\prec$ " is a transitive relation of this space.

4. $f_{K_{1} \cdot K_{2}}=f_{K_{1}} \circ f_{K_{2}}$ in $\mathbb{H} ; g_{K_{1} \cdot K_{2}}=g_{K_{2}} \circ g_{K_{1}}$ in $\mathbb{H} \backslash\left(K_{1} \cdot K_{2}\right)$.

5. hcap $\left(K_{1} \cdot K_{2}\right)=\operatorname{hcap}\left(K_{1}\right)+\operatorname{hcap}\left(K_{2}\right)$. If $K_{1} \subset K_{2}$ or $K_{1} \prec K_{2}$, then hcap $\left(K_{1}\right) \leq$ $\operatorname{hcap}\left(K_{2}\right)$.

From $f_{K_{1} \cdot K_{2}}=f_{K_{1}} \circ f_{K_{2}}$ in $\mathbb{H}$ we can conclude that $f_{K_{1}}=f_{K_{1} \cdot K_{2}} \circ g_{K_{2}}$ in $\mathbb{H} \backslash K_{2}$. So $f_{K_{1}}$ is an analytic extension of $f_{K_{1} \cdot K_{2}} \circ g_{K_{2}}$, which means that $K_{1}$ is uniquely determined by $K_{1} \cdot K_{2}$ and $K_{2}$. So the following definition makes sense.

Definition 2.5. Let $K_{1}$ and $K_{2}$ be $\mathbb{H}$-hulls such that $K_{1} \prec K_{2}$. We use $K_{2}: K_{1}$ to denote the unique $\mathbb{H}$-hull $K \subset K_{2}$ such that $K_{2} / K=K_{1}$.

For an $\mathbb{H}$-hull $K$, the base of $K$ is the set $B_{K}=\bar{K} \cap \mathbb{R}$. Let the double of $K$ be defined by $\widehat{K}=K \cup I_{\mathbb{R}}(K) \cup B_{K}$, where $I_{\mathbb{R}}(z):=\bar{z}$. Then $g_{K}$ extends to a conformal map (still denoted by $\left.g_{K}\right)$ in $\widehat{\mathbb{C}} \backslash \widehat{K}$, which satisfies $g_{K}(\infty)=\infty, g_{K}^{\prime}(\infty)=1$, and $g_{K} \circ I_{\mathbb{R}}=I_{\mathbb{R}} \circ g_{K}$. Moreover, $g_{K}(\widehat{\mathbb{C}} \backslash \widehat{K})=\widehat{\mathbb{C}} \backslash S_{K}$ for some compact $S_{K} \subset \mathbb{R}$, which is called the support of $K$. So $f_{K}$ extends to a conformal map from $\widehat{\mathbb{C}} \backslash S_{K}$ onto $\widehat{\mathbb{C}} \backslash \widehat{K}$.

Lemma 2.6. $f_{K}$ can not be extended analytically beyond $\widehat{\mathbb{C}} \backslash S_{K}$.

Proof. Suppose $f_{K}$ can be extended analytically near $x_{0} \in \mathbb{R}$, then the image of $f_{K}$ contains a neighborhood of $f_{K}\left(x_{0}\right) \in \mathbb{R}$. So $f_{K}(\mathbb{H})=\mathbb{H} \backslash K$ contains a neighborhood of $f_{K}\left(x_{0}\right)$ in $\mathbb{H}$. This then implies that $f_{K}\left(x_{0}\right) \in \mathbb{R} \backslash B_{K}$. Thus, there is $y_{0} \in \mathbb{R} \backslash S_{K}$ such that $f_{K}\left(y_{0}\right)=f_{K}\left(x_{0}\right)$. Since $f_{K}$ is conformal in $\mathbb{H}$, we must have $x_{0}=y_{0} \in \mathbb{R} \backslash S_{K}$.

Lemma 2.7. If $K_{1}=K_{2} / K_{0} \prec K_{2}$, then $S_{K_{1}} \subset S_{K_{2}}, f_{K_{2}}=f_{K_{0}} \circ f_{K_{1}}$ in $\widehat{\mathbb{C}} \backslash S_{K_{2}}$, and $g_{K_{2}}=g_{K_{1}} \circ g_{K_{0}}$ in $\widehat{\mathbb{C}} \backslash \widehat{K}_{2}$.

Proof. Since $K_{2}=K_{0} \cdot K_{1}$, we have $f_{K_{2}}=f_{K_{0}} \circ f_{K_{1}}$ in $\mathbb{H}$, which implies that $g_{K_{0}} \circ f_{K_{2}}=f_{K_{1}}$ in $\mathbb{H}$. Since $f_{K_{2}}$ maps $\widehat{\mathbb{C}} \backslash S_{K_{2}}$ conformally onto $\widehat{\mathbb{C}} \backslash \widehat{K}_{2} \subset \widehat{\mathbb{C}} \backslash \widehat{K}_{0}$, and $g_{K_{0}}$ is analytic in $\widehat{\mathbb{C}} \backslash \widehat{K}_{2}$, we see that $g_{K_{0}} \circ f_{K_{2}}$ is analytic in $\widehat{\mathbb{C}} \backslash S_{K_{2}}$. Since $g_{K_{0}} \circ f_{K_{2}}=f_{K_{1}}$ in $\mathbb{H}$, from Lemma 2.6 we have $S_{K_{1}} \subset S_{K_{2}}$, and $g_{K_{0}} \circ f_{K_{2}}=f_{K_{1}}$ in $\widehat{\mathbb{C}} \backslash S_{K_{2}}$. Composing $f_{K_{0}}$ to the left of both sides, we get $f_{K_{2}}=f_{K_{0}} \circ f_{K_{1}}$ in $\widehat{\mathbb{C}} \backslash S_{K_{2}}$. Taking inverse, we obtain the equality for $g_{K}$ 's.

Definition 2.8. $S \subset \widehat{\mathbb{C}}$ is called $\mathbb{R}$-symmetric if $I_{\mathbb{R}}(S)=S$. An $\mathbb{R}$-symmetric map $W$ is a function defined in an $\mathbb{R}$-symmetric domain $\Omega$, which commutes with $I_{\mathbb{R}}$, and maps $\Omega \cap \mathbb{H}$ into $\mathbb{H}$.

\section{Remarks.}

1. For any $\mathbb{H}$-hull $K, g_{K}$ and $f_{K}$ are $\mathbb{R}$-symmetric conformal maps.

2. Let $W$ be an $R$-symmetric conformal map defined in $\Omega$. If an $\mathbb{H}$-hull $K$ satisfies $\widehat{K} \subset \Omega$ and $\infty \notin W(\widehat{K})$, then $W(K)$ is also an $\mathbb{H}$-hull and $\widehat{W(K)}=W(\widehat{K})$.

Definition 2.9. Let $\Omega$ be an $\mathbb{R}$-symmetric domain and $K$ be an $\mathbb{H}$-hull. If $\widehat{K} \subset \Omega$, we write $\Omega_{K}$ or $(\Omega)_{K}$ for $S_{K} \cup g_{K}(\Omega \backslash \widehat{K})$, and call it the collapse of $\Omega$ via $K$. If $S_{K} \subset \Omega$, we write $\Omega^{K}$ or $\left(\Omega^{K}\right)$ for $\widehat{K} \cup f_{K}\left(\Omega \backslash S_{K}\right)$, and call it the lift of $\Omega$ via $K$. 


\section{Remarks.}

1. In the definition, $\Omega_{K}$ is an $\mathbb{R}$-symmetric domain containing $S_{K} ; \Omega^{K}$ is an $\mathbb{R}$-symmetric domain containing $\widehat{K}$.

2. $\left(\Omega_{K}\right)^{K}=\Omega$ and $\left(\Omega^{K}\right)_{K}=\Omega$ if the lefthand sides are well defined.

3. $\Omega_{K_{1} \cdot K_{2}}=\left(\Omega_{K_{1}}\right)_{K_{2}}$ and $\Omega^{K_{1} \cdot K_{2}}=\left(\Omega^{K_{2}}\right)^{K_{1}}$ if either sides are well defined.

Definition 2.10. Let $W$ be an $\mathbb{R}$-symmetric conformal map with domain $\Omega$. Let $K$ be an $\mathbb{H}$-hull such that $\widehat{K} \subset \Omega$ and $\infty \notin W(\widehat{K})$. We write $W_{K}$ or $(W)_{K}$ for the conformal extension of $g_{W(K)} \circ W \circ f_{K}$ to $\Omega_{K}$, and call it the collapse of $W$ via $K$.

\section{Remarks.}

1. Since $g_{W(K)} \circ W \circ f_{K}: \Omega_{K} \backslash S_{K} \stackrel{\text { Conf }}{\rightarrow} W(\Omega) \backslash S_{W(K)}$, the existence of $W_{K}$ follows from the Schwarz reflection principle. $W_{K}$ is an $\mathbb{R}$-symmetric conformal map, and $W_{K}\left(S_{K}\right)=S_{W(K)}$.

2. The $g_{K}$ and $f_{K}$ defined at the beginning of this section should not be understood as the collapse of $g$ and $f$ via $K$.

3. $W_{K_{1} \cdot K_{2}}=\left(W_{K_{1}}\right)_{K_{2}}$ if either side is well defined.

4. $V_{W(K)} \circ W_{K}=(V \circ W)_{K}$ if either side is well defined. In particular, $\left(W^{-1}\right)_{W(K)}=$ $\left(W_{K}\right)^{-1}$.

Let $B_{K}^{*}$ and $S_{K}^{*}$ be the convex hulls of $B_{K}$ and $S_{K}$, respectively. Let $\widehat{K}^{*}=\widehat{K} \cup B_{K}^{*}$. Then $g_{K}: \widehat{\mathbb{C}} \backslash \widehat{K}^{*} \stackrel{\text { Conf }}{\rightarrow} \widehat{\mathbb{C}} \backslash S_{K}^{*}$. If $K \neq \emptyset$, then $S_{K}^{*}$ is a bounded closed interval, $\widehat{K}^{*}$ is a nondegenerate interior hull, and $\psi_{\widehat{K}^{*}}=f_{K} \circ \psi_{S_{K}^{*}}$. If $S_{K}^{*} \subset \Omega$, then $\Omega^{K}=\widehat{K}^{*} \cup f_{K}\left(\Omega \backslash S_{K}^{*}\right)$. The lemma below is a part of Lemma 5.3 in [17], where $S_{K}^{*}$ was denoted by $\left[c_{K}, d_{K}\right]$.

Lemma 2.11. If $K_{1} \subset K_{2}$, then $S_{K_{1}}^{*} \subset S_{K_{2}}^{*}$.

Theorem 2.12. Let $W$ be an $\mathbb{R}$-symmetric conformal map with domain $\Omega$. Let $K$ be an $\mathbb{H}$-hull such that $S_{K} \subset \Omega$ and $\infty \notin W\left(S_{K}\right)$. Then there is a unique $\mathbb{R}$-symmetric conformal map $V$ defined in $\Omega^{K}$ such that $V_{K}=W$.

Proof. We first consider the existence. If $K=\emptyset$, since $f_{\emptyset}=$ id and $\Omega^{\emptyset}=\Omega, V=W$ is what we need. Now suppose $K \neq \emptyset$ and $S_{K}^{*} \subset \Omega$. Note that $S_{K}^{*}$ is a bounded closed interval, and so is $W\left(S_{K}^{*}\right)$. Let $\Omega_{\mathbb{T}}=\psi_{S_{K}^{*}}^{-1}\left(\Omega \backslash S_{K}^{*}\right)$. Define a conformal map $W_{\mathbb{T}}$ in $\Omega_{\mathbb{T}}$ by $W_{\mathbb{T}}=\psi_{W\left(S_{K}^{*}\right)}^{-1} \circ W \circ \psi_{S_{K}^{*}}$. Then $W_{\mathbb{T}}(z) \rightarrow \mathbb{T}$ as $\Omega_{\mathbb{T}} \ni z \rightarrow \mathbb{T}$. Thus, $W_{\mathbb{T}}$ extends conformally across $\mathbb{T}$, maps $\mathbb{T}$ onto $\mathbb{T}$, and preserves the orientation of $\mathbb{T}$. Apply Theorem 2.2 to $W_{\mathbb{T}}$ and $\widehat{K}^{*}$. We find a conformal map $\widehat{V}$ defined in

$$
\widehat{K}^{*} \cup \psi_{\widehat{K}^{*}}\left(\Omega_{\mathbb{T}}\right)=\widehat{K}^{*} \cup f_{K}\left(\Omega \backslash S_{K}^{*}\right)=\Omega^{K}
$$

such that $\widehat{V} \circ \psi_{\widehat{K}^{*}}=\psi_{\widehat{V}\left(\widehat{K}^{*}\right)} \circ W_{\mathbb{T}}$ in $\Omega_{\mathbb{T}}$. Let $\widetilde{V}=I_{\mathbb{R}} \circ \widehat{V} \circ I_{\mathbb{R}}$. Then $\widetilde{V}\left(\widehat{K}^{*}\right)=I_{\mathbb{R}} \circ \widehat{V}\left(\widehat{K}^{*}\right)$. So $\psi_{\widetilde{V}\left(\widehat{K}^{*}\right)}=I_{\mathbb{R}} \circ \psi_{\widehat{V}\left(\widehat{K}^{*}\right)} \circ I_{\mathbb{R}}$. Since $I_{\mathbb{R}}$ commutes with $\psi_{\widehat{K}^{*}}$ and $W_{\mathbb{T}}$, we see that $\widetilde{V}$ also satisfies the properties of $\widehat{V}$. So $\widetilde{V}=a \widehat{V}+b$ for some $a>0$ and $b \in \mathbb{C}$. Thus, $I_{\mathbb{R}} \circ \widehat{V} \circ I_{\mathbb{R}}=a \widehat{V}+b$. Considering the values of $\widehat{V}$ on $\Omega^{K} \cap \mathbb{R}$, we find that $a=1$ and $\operatorname{Re} b=0$. Note that $\widehat{V}-\frac{b}{2}$ satisfies the property of $\widehat{V}$, and commutes with $I_{\mathbb{R}}$. By replacing $\widehat{V}$ with $\widehat{V}-\frac{b}{2}$, we may assume that $\widehat{V}$ is an $\mathbb{R}$-symmetric conformal map. 


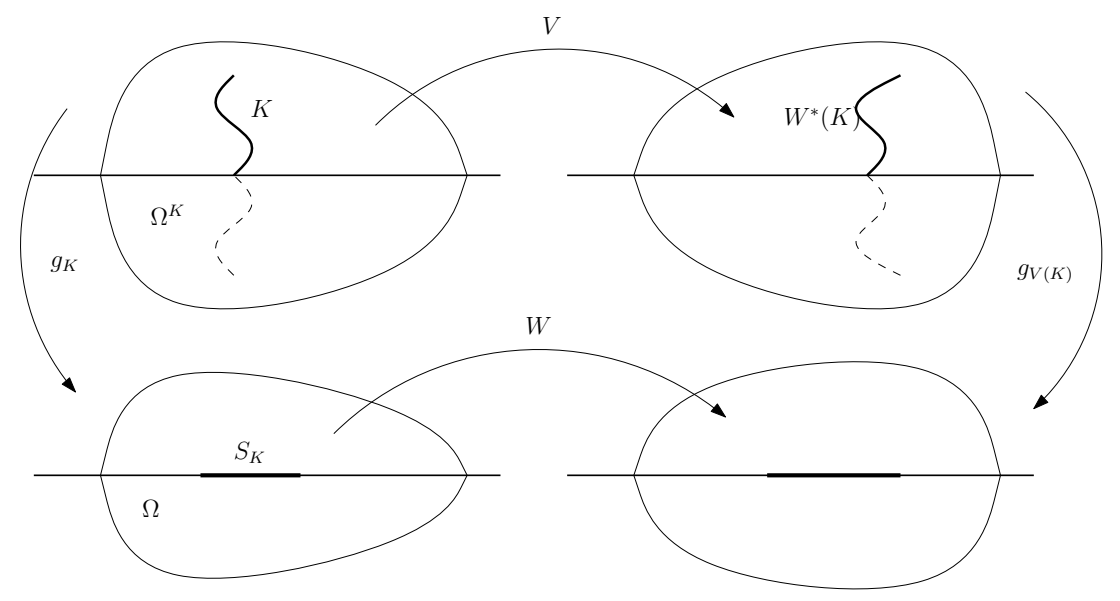

Figure 2: The situation of Theorem 2.12, Given $K$ and $W$, there is a unique $V$, also denoted $W^{K}$, which is analytic across $K$ and its reflection $I_{\mathbb{R}}(K)$, see Definition 2.13.

Since $\widehat{V} \circ \psi_{\widehat{K}^{*}}=\psi_{\widehat{V}\left(\widehat{K}^{*}\right)} \circ W_{\mathbb{T}}$ in $\Omega_{\mathbb{T}}$, from $\psi_{\widehat{K}^{*}}=f_{K} \circ \psi_{S_{K}^{*}}, \psi_{\widehat{V}\left(\widehat{K}^{*}\right)}=f_{\widehat{V}(K)} \circ \psi_{S_{\widehat{V}(K)}^{*}}$, and the definitions of $W_{\mathbb{T}}$ and $\Omega_{\mathbb{T}}$, we have

$$
\widehat{V} \circ f_{K}=f_{\widehat{V}(K)} \circ \psi_{S_{\widehat{V}(K)}^{*}} \circ \psi_{W\left(S_{K}^{*}\right)}^{-1} \circ W
$$

on $\Omega \backslash S_{K}^{*}$. Let $h=\psi_{S_{\widehat{V}(K)}^{*}} \circ \psi_{W\left(S_{K}^{*}\right)}^{-1}$. Since $S_{\widehat{V}(K)}^{*}$ and $W\left(S_{K}^{*}\right)$ are both bounded closed intervals, we have $h(z)=a z+b$ for some $a>0$ and $b \in \mathbb{R}$. Let $V=h^{-1} \circ \widehat{V}$. Then $V$ is also an $\mathbb{R}$-symmetric conformal map defined on $\Omega^{K}$, and $f_{V(K)}=h^{-1} \circ f_{\widehat{V}(K)} \circ h$. From 2.1 we have

$$
f_{V(K)} \circ W=h^{-1} \circ f_{\widehat{V}(K)} \circ h \circ W=h^{-1} \circ \widehat{V} \circ f_{K}=V \circ f_{K} .
$$

This finishes the existence part in the case that $K \neq \emptyset$ and $S_{K}^{*} \subset \Omega$.

Now we still assume that $K \neq \emptyset$ but do not assume that $S_{K}^{*} \subset \Omega$. Let $\Omega_{0}=\Omega$ and $W_{0}=W$. We will construct $\mathbb{H}$-hulls $K_{1}, \ldots, K_{n}$ and $\mathbb{R}$-symmetric domains $\Omega_{1}, \ldots, \Omega_{n}$ such that $K_{n} \cdot K_{n-1} \cdots K_{1}=K, \Omega_{j}=\Omega_{j-1}^{K_{j}}$, and $S_{K_{j}}^{*} \subset \Omega_{j-1}, 1 \leq j \leq n$. When they are constructed, using the above result, we can obtain $\mathbb{R}$-symmetric conformal maps $W_{j}$ defined on $\Omega_{j}, 1 \leq j \leq n$, such that $\left(W_{j}\right)_{K_{j}}=W_{j-1}, 1 \leq j \leq n$. Let $V=W_{n}$. Then $V$ is defined in $\Omega_{n}=\Omega_{0}^{K_{n} \cdots K_{1}}=\Omega^{K}$, and $V_{K}=\left(W_{n}\right)_{K_{n} \cdots K_{1}}=W_{0}=W$. So $V$ is what we need.

It remains to construct $K_{j}$ and $\Omega_{j}$ with the desired properties. Since $\Omega \cap \mathbb{R}$ is a disjoint union of open intervals, and $S_{K}$ is a compact subset of $\Omega \cap \mathbb{R}$, we may find finitely many components of $\Omega \cap \mathbb{R}$ which cover $S_{K}$. There exist mutually disjoint $\mathbb{R}$-symmetric Jordan curves $J_{1}, \ldots, J_{n}$ in $\Omega$ such that their interiors $D_{J_{1}}, \ldots, D_{J_{n}}$ are mutually disjoint and contained in $\Omega$, and $S_{K} \subset \bigcup_{k=1}^{n} D_{J_{k}}$. Then $J_{j}^{K}:=f_{K}\left(J_{j}\right), 1 \leq j \leq n$ are $\mathbb{R}$-symmetric Jordan curves, which together with their interiors are mutually disjoint, and $\widehat{K} \subset \bigcup_{k=1}^{n} D_{f_{K}\left(J_{k}\right)}$. Let $H_{j}=K \cap \bigcup_{k=j}^{n} D_{J_{j}^{K}}, 1 \leq j \leq n$. Then each $H_{j}$ is an $\mathbb{H}$-hull, and $K=H_{1} \supset H_{2} \supset \cdots \supset H_{n}$. Let $K_{j}=H_{j} / H_{j+1}, 1 \leq j \leq n-1$, and $K_{n}=H_{n}$. Then we have $K_{n} \cdots K_{1}=H_{1}=K$. 
Construct $\Omega_{j}, 1 \leq j \leq n$, such that $\Omega_{j}=\Omega_{j-1}^{K_{j}}, 1 \leq j \leq n$. Then

$$
\Omega_{j-1}=\left(\Omega_{0}\right)^{K_{j-1} \cdots K_{1}}=\left(\Omega^{K_{n} \cdots K_{1}}\right)_{K_{n} \cdots K_{j}}=\left(\Omega^{K}\right)_{H_{j}}, \quad 1 \leq j \leq n .
$$

It suffices to show that $S_{K_{j}}^{*} \subset \Omega_{j-1}$. We have

$$
K_{j}=H_{j} / H_{j+1}=g_{H_{j+1}}\left(H_{j} \backslash H_{j+1}\right)=g_{H_{j+1}}\left(K \cap D_{J_{j}^{K}}\right) .
$$

Thus, $\widehat{K}_{j} \subset D_{g_{H_{j+1}}\left(J_{j}^{K}\right)}$, which implies that $S_{K_{j}} \subset D_{g_{H_{j}}\left(J_{j}^{K}\right)}$. Since $\mathbb{R} \cap D_{g_{H_{j}}\left(J_{j}^{K}\right)}$ is an interval, we have $S_{K_{j}}^{*} \subset D_{g_{H_{j}}\left(J_{j}^{K}\right)}$. Since $\overline{D_{J_{j}^{K}}} \subset \Omega^{K}$, and $J_{j}^{K}$ has positive distance from $H_{j}$, we have $D_{g_{H_{j}}\left(J_{j}^{K}\right)} \subset\left(\Omega^{K}\right)_{H_{j}}=\Omega_{j-1}$. So $K_{j}$ and $\Omega_{j}$ satisfy the properties we need. This finishes the proof of the existence part.

Now we prove the uniqueness. Suppose $\widetilde{V}$ is another $\mathbb{R}$-symmetric conformal map defined on $\Omega^{K}$ such that $\widetilde{V}_{K}=W$. Then

$$
g_{V(K)} \circ V=W \circ g_{K}=g_{\widetilde{V}(K)} \circ \widetilde{V}
$$

on $\Omega \backslash \widehat{K}$. Thus, $\widetilde{V} \circ V^{-1}=f_{\widetilde{V}(K)} \circ g_{V(K)}$ on $V(\Omega \backslash \widehat{K})=V(\Omega) \backslash V(\widehat{K})$. We know that $\widetilde{V} \circ V^{-1}$ is a conformal map defined on $V(\Omega)$, while $f_{\widetilde{V}(K)} \circ g_{V(K)}$ is a conformal map defined on $\widehat{\mathbb{C}} \backslash \widehat{V(K)}=\widehat{C} \backslash V(\widehat{K})$. Since $V(\Omega)$ and $\widehat{\mathbb{C}} \backslash V(\widehat{K})$ cover $\widehat{C}$, we may define an analytic function $h$ on $\mathbb{C}$ such that $h=\widetilde{V} \circ V^{-1}$ on $V(\Omega)$ and $h=f_{\widetilde{V}(K)} \circ g_{V(K)}$ on $\widehat{\mathbb{C}} \backslash V(\widehat{K})$. From the properties of $f_{\widetilde{V}(K)}$ and $g_{V(K)}$, we see that $h(z)-z \rightarrow 0$ as $z \rightarrow \infty$. So $h=$ id, which implies that $\widetilde{V}=V$. So the uniqueness is proved.

Definition 2.13. We use $W^{K}$ to denote the unique $V$ in Theorem 2.12, and call it the lift of $W$ via $K$. Let $W^{*}$ be the map defined by $W^{*}(K)=W^{K}(K)$.

\section{Remarks.}

1. $\left(W_{K}\right)^{K}=W$ and $\left(W^{K}\right)_{K}=W$.

2. The range of $W^{K}$ is $W^{K}\left(\Omega^{K}\right)=(W(\Omega))^{W^{*}(K)}$.

3. $W^{K_{1} \cdot K_{2}}=\left(W^{K_{2}}\right)^{K_{1}}, V^{W^{K}(K)} \circ W^{K}=(V \circ W)^{K}$, and $\left(W^{K}\right)^{-1}=\left(W^{-1}\right)^{W(K)}$.

4. The domain (resp. range) of $W^{*}$ is the set of $\mathbb{H}$-hulls whose supports are contained in the domain (resp. range) of $W$; and $S_{W^{*}(K)}=W\left(S_{K}\right)$.

5. $V^{*} \circ W^{*}=(V \circ W)^{*} ;\left(W^{*}\right)^{-1}=\left(W^{-1}\right)^{*}$.

Lemma 2.14. Suppose $K_{1} \prec K_{2}, S_{K_{2}}$ lies in the domain of an $\mathbb{R}$-symmetric conformal map $W$, and $\infty \notin W\left(S_{K_{2}}\right)$. Then $W^{*}\left(K_{1}\right) \prec W^{*}\left(K_{2}\right)$, and

$$
W^{*}\left(K_{2}\right): W^{*}\left(K_{1}\right)=W^{K_{2}}\left(K_{2}: K_{1}\right) .
$$

Proof. From Lemma 2.7, $S_{K_{1}} \subset S_{K_{2}}$. So $W^{K_{1}}$ and $W^{K_{2}}$ exist. Let $K_{0}=K_{2}: K_{1} \subset K_{2}$. Then $W^{K_{2}}\left(K_{0}\right) \subset W^{K_{2}}\left(K_{2}\right)$ and

$$
W^{K_{2}}\left(K_{2}\right) / W^{K_{2}}\left(K_{0}\right)=g_{W^{K_{2}}\left(K_{0}\right)} \circ W^{K_{2}}\left(K_{2} \backslash K_{0}\right)
$$




$$
\begin{gathered}
=g_{W^{K_{2}}\left(K_{0}\right)} \circ W^{K_{2}} \circ f_{K_{0}}\left(K_{2} / K_{0}\right)=\left(W^{K_{2}}\right)_{K_{0}}\left(K_{2} / K_{0}\right) \\
=\left(W^{K_{0} \cdot K_{1}}\right)_{K_{0}}\left(K_{1}\right)=W^{K_{1}}\left(K_{1}\right) .
\end{gathered}
$$

Thus, $W^{K_{1}}\left(K_{1}\right) \prec W^{K_{2}}\left(K_{2}\right)$ and $W^{K_{2}}\left(K_{2}\right): W^{K_{1}}\left(K_{1}\right)=W^{K_{2}}\left(K_{0}\right)$.

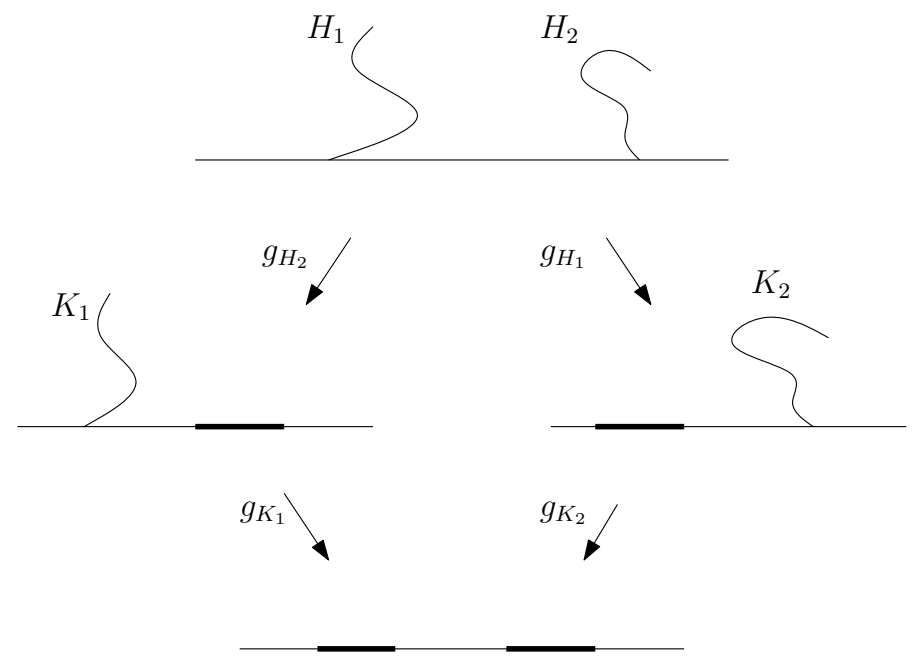

Figure 3: The pair $\left(H_{1}, H_{2}\right)$ uniquely determines the pair $\left(K_{1}, K_{2}\right)$, and vice versa, see Theorem 2.16 and Definition 2.15 .

Definition 2.15. Let $\mathcal{P}^{*}$ denote the set of pair of $\mathbb{H}$-hulls $\left(H_{1}, H_{2}\right)$ such that $\widehat{H}_{1} \cap \widehat{H}_{2}=\emptyset$. Let $\mathcal{P}_{*}$ denote the set of pair of $\mathbb{H}$-hulls $\left(K_{1}, K_{2}\right)$ such that $S_{K_{1}} \cap S_{K_{2}}=\emptyset$. Define $g_{*}$ on $\mathcal{P}^{*}$ by $g_{*}\left(H_{1}, H_{2}\right)=\left(g_{H_{2}}\left(H_{1}\right), g_{H_{1}}\left(H_{2}\right)\right)$. Define $f^{*}$ on $\mathcal{P}_{*}$ by $f^{*}\left(K_{1}, K_{2}\right)=\left(f_{K_{2}}^{*}\left(K_{1}\right), f_{K_{1}}^{*}\left(K_{2}\right)\right)$.

\section{Remarks.}

1. $g_{*}$ is well defined on $\mathcal{P}^{*}$ because for $j=1,2, \widehat{K}_{3-j}$ is contained in the domain of $g_{K_{j}}$ : $\widehat{\mathbb{C}} \backslash \widehat{K}_{j}$. The value of $g_{*}$ is a pair of $\mathbb{H}$-hulls.

2. $f^{*}$ is well defined on $\mathcal{P}_{*}$ because for $j=1,2, S_{K_{3-j}}$ is contained in the domain of $f_{K_{j}}$ : $\widehat{\mathbb{C}} \backslash S_{K_{j}}$. The value of $f^{*}$ is a pair of $\mathbb{H}$-hulls.

Theorem 2.16. $g_{*}$ and $f^{*}$ are bijections between $\mathcal{P}^{*}$ and $\mathcal{P}_{*}$, and are inverse of each other. Moreover, if $\left(H_{1}, H_{2}\right)=f^{*}\left(K_{1}, K_{2}\right)$, then

(i) $H_{1} \cdot K_{2}=H_{2} \cdot K_{1}=H_{1} \cup H_{2}$;

(ii) $f_{K_{2}}\left(S_{K_{1}}\right)=S_{H_{1}}$ and $f_{K_{1}}\left(S_{K_{2}}\right)=S_{H_{2}}$;

(iii) $S_{H_{1} \cup H_{2}}=S_{K_{1}} \cup S_{K_{2}}$.

Proof. Let $\left(H_{1}, H_{2}\right) \in \mathcal{P}^{*}$ and $\left(K_{1}, K_{2}\right)=g_{*}\left(H_{1}, H_{2}\right)$. Then $\left(\widehat{\mathbb{C}} \backslash \widehat{H}_{1}\right)_{H_{2}}=\widehat{\mathbb{C}} \backslash \widehat{K}_{1}, S_{H_{1}} \subset$ $\widehat{\mathbb{C}} \backslash \widehat{K}_{2}$, and $\left(\widehat{\mathbb{C}} \backslash S_{H_{1}}\right)_{K_{2}}=\widehat{\mathbb{C}} \backslash g_{K_{2}}\left(S_{H_{1}}\right)$. Since $g_{H_{1}}: \widehat{\mathbb{C}} \backslash \widehat{H}_{1} \stackrel{\text { Conf }}{\rightarrow} \widehat{\mathbb{C}} \backslash S_{H_{1}}$ and $g_{H_{1}}\left(H_{2}\right)=K_{2}$, 
we get $\left(g_{H_{1}}\right)_{H_{2}}: \widehat{\mathbb{C}} \backslash \widehat{K}_{1} \stackrel{\text { Conf }}{\rightarrow} \widehat{\mathbb{C}} \backslash g_{K_{2}}\left(S_{H_{1}}\right)$. From the normalization of $g_{H_{1}}, g_{H_{2}}, g_{K_{2}}$ at $\infty$, we conclude that

$$
\left(g_{H_{1}}\right)_{H_{2}}=g_{K_{1}}, \quad g_{K_{2}}\left(S_{H_{1}}\right)=S_{K_{1}} .
$$

From $S_{H_{1}} \subset \widehat{\mathbb{C}} \backslash \widehat{K}_{2}$ and $g_{K_{2}}\left(S_{H_{1}}\right)=S_{K_{1}}$, we see that $S_{K_{1}} \cap S_{K_{2}}=\emptyset$, i.e., $\left(K_{1}, K_{2}\right) \in \mathcal{P}_{*}$. Since $f_{H_{1}}=g_{H_{1}}^{-1}, f_{K_{1}}=g_{K_{1}}^{-1}$, and $g_{H_{1}}\left(H_{2}\right)=K_{2}$, from 2.3 we get $\left(f_{H_{1}}\right)_{K_{2}}=f_{K_{1}}$, which implies that $\left(f_{K_{1}}\right)^{K_{2}}=f_{H_{1}}$. Thus, $f_{K_{1}}^{*}\left(K_{2}\right)=f_{H_{1}}\left(K_{2}\right)=H_{2}$. Similarly, $f_{K_{2}}^{*}\left(K_{1}\right)=H_{1}$. Thus, $f^{*}\left(K_{1}, K_{2}\right)=\left(H_{1}, H_{2}\right)$. So $f^{*} \circ g_{*}=\operatorname{id}_{\mathcal{P}^{*}}$.

Let $\left(K_{1}, K_{2}\right) \in \mathcal{P}_{*}$ and $H_{1}=f_{K_{2}}^{*}\left(K_{1}\right)$. Then $S_{H_{1}}=f_{K_{2}}\left(S_{K_{1}}\right)$ is disjoint from $\widehat{K}_{2}$. Thus, we may define another $\mathbb{H}$-hull $H_{2}:=f_{H_{1}}\left(K_{2}\right)$. Then $\widehat{H}_{2} \subset \widehat{\mathbb{C}} \backslash \widehat{H}_{1}$. So $\left(H_{1}, H_{2}\right) \in \mathcal{P}^{*}$. We have $\left(\widehat{\mathbb{C}} \backslash S_{K_{2}}\right)^{K_{1}}=\widehat{\mathbb{C}} \backslash f_{K_{1}}\left(S_{K_{2}}\right)$ and $\left(\widehat{\mathbb{C}} \backslash \widehat{K}_{2}\right)^{H_{1}}=\widehat{\mathbb{C}} \backslash \widehat{H}_{2}$. Since $f_{K_{2}}: \widehat{\mathbb{C}} \backslash S_{K_{2}} \stackrel{\text { Conf }}{\rightarrow} \widehat{\mathbb{C}} \backslash \widehat{K}_{2}$ and $\left(f_{K_{2}}\right)^{*}\left(K_{1}\right)=H_{1}$, we see that $\left(f_{K_{2}}\right)^{K_{1}}: \widehat{\mathbb{C}} \backslash f_{K_{1}}\left(S_{K_{2}}\right) \stackrel{\text { Conf }}{\rightarrow} \widehat{\mathbb{C}} \backslash \widehat{H}_{2}$. From the normalization of $f_{K_{1}}, f_{H_{1}}, f_{K_{2}}$ at $\infty$, we conclude that

$$
\left(f_{K_{2}}\right)^{K_{1}}=f_{H_{2}}, \quad f_{K_{1}}\left(S_{K_{2}}\right)=S_{H_{2}} .
$$

Since $H_{1}=f_{K_{2}}^{*}\left(K_{1}\right)$, we get $f_{K_{2}}=g_{H_{1}} \circ f_{H_{2}} \circ f_{K_{1}}$ on $\left(\widehat{\mathbb{C}} \backslash S_{K_{2}}\right) \backslash S_{K_{1}}$, which implies that $f_{H_{1}} \circ f_{K_{2}}=f_{H_{2}} \circ f_{K_{1}}$ on $\widehat{\mathbb{C}} \backslash\left(S_{K_{1}} \cup S_{K_{2}}\right)$. So

$$
H_{2} \cdot K_{1}=H_{1} \cdot K_{2}=H_{1} \cup f_{H_{1}}\left(K_{2}\right)=H_{1} \cup H_{2} .
$$

Thus, $K_{1}=g_{H_{2}}\left(H_{1}\right)$ and $K_{2}=g_{H_{1}}\left(H_{2}\right)$, i.e., $\left(K_{1}, K_{2}\right)=g_{*}\left(H_{1}, H_{2}\right)$. This shows that the range of $g_{*}$ is $\mathcal{P}_{*}$, which combining with $f^{*} \circ g_{*}=\operatorname{id}_{\mathcal{P}^{*}}$ shows that $f^{*}=\left(g_{*}\right)^{-1}$ and $g_{*}=\left(f^{*}\right)^{-1}$.

In the previous paragraph, since $\left(K_{1}, K_{2}\right)=g_{*}\left(H_{1}, H_{2}\right), f^{*}\left(K_{1}, K_{2}\right)=\left(H_{1}, H_{2}\right)$. Thus, (i) follows from (2.5); the second parts of (ii) follow from (2.4), and the first part follows from symmetry. Finally, since $g_{K_{2}} \circ g_{H_{1}}=g_{H_{1} \cdot K_{2}}=g_{H_{1} \cup H_{2}}$, from $g_{H_{1}}: \widehat{\mathbb{C}} \backslash\left(\widehat{H}_{1} \cup \widehat{H}_{2}\right) \stackrel{\text { Conf }}{\rightarrow}$ $\widehat{\mathbb{C}} \backslash\left(S_{H_{1}} \cup \widehat{K}_{2}\right), g_{K_{2}}: \widehat{\mathbb{C}} \backslash\left(S_{H_{1}} \cup \widehat{K}_{2}\right) \stackrel{\operatorname{Conf}}{\rightarrow} \widehat{\mathbb{C}} \backslash\left(g_{K_{2}}\left(S_{H_{1}}\right) \cup S_{K_{2}}\right)$, and 2.3), we get (iii).

Definition 2.17. For $\left(K_{1}, K_{2}\right) \in \mathcal{P}_{*}$, we define the quotient union of $K_{1}$ and $K_{2}$ to be $K_{1} \vee K_{2}=H_{1} \cup H_{2}$, where $\left(H_{1}, H_{2}\right)=f^{*}\left(K_{1}, K_{2}\right)$.

Remark. From Theorem 2.16, $K_{1}, K_{2} \prec K_{1} \vee K_{2}$ and $S_{K_{1} \vee K_{2}}=S_{K_{1}} \cup S_{K_{2}}$.

The space of $\mathbb{H}$-hulls has a natural metric $d_{\mathcal{H}}$ described in Appendix $\mathrm{C}$ Let $\mathcal{H}_{S}$ denote the set of $\mathbb{H}$-hulls whose supports are contained in $S$. From Lemma C.2 if $F$ is compact, $\left(\mathcal{H}_{F}, d_{\mathcal{H}}\right)$ is compact, and $H_{n} \rightarrow H$ in $\mathcal{H}_{F}$ implies that $f_{H_{n}} \stackrel{\text { l.u. }}{\longrightarrow} f_{H}$ in $\mathbb{C} \backslash F$.

Theorem 2.18. (i) Let $F \subset \mathbb{R}$ be compact. Let $W$ be an $\mathbb{R}$-symmetric conformal map whose domain contains $F$. Then $W^{*}: \mathcal{H}_{F} \rightarrow \mathcal{H}_{W(F)}$ is continuous.

(ii) Let $E$ and $F$ be two nonempty compact subsets of $\mathbb{R}$ with $E \cap F=\emptyset$. Then $f^{*}$ and $\left(K_{1}, K_{2}\right) \mapsto K_{1} \vee K_{2}$ are continuous on $\mathcal{H}_{E} \times \mathcal{H}_{F}$.

Proof. (i). First, $W^{*}$ is well defined on $\mathcal{H}_{F}$, and the range of $W^{*}$ is $\mathcal{H}_{W(F)}$. Suppose $\left(H_{n}\right)$ is a sequence in $\mathcal{H}_{F}$ and $H_{n} \rightarrow H_{0} \in \mathcal{H}_{F}$. To prove the continuity of $W^{*}$, we need to show that $W^{*}\left(H_{n}\right) \rightarrow W^{*}\left(H_{0}\right)$. Suppose this is not true. Since $\mathcal{H}_{W(F)}$ is compact, by passing to a subsequence, we may assume that $W^{*}\left(H_{n}\right) \rightarrow K_{0} \neq W^{*}\left(H_{0}\right)$. For each $n_{k}$, 
$W^{H_{n_{k}}}=f_{W^{*}\left(H_{n_{k}}\right)} \circ W \circ g_{H_{n_{k}}}$ on $f_{H_{n_{k}}}(\Omega \backslash F)$. We have $g_{H_{n_{k}}} \stackrel{\text { l.u. }}{\longrightarrow} g_{H_{0}}$ in $f_{H_{0}}(\Omega \backslash F)$ and $f_{W^{*}\left(H_{n_{k}}\right)} \stackrel{\text { l.u. }}{\longrightarrow} f_{K_{0}}$ in $W(\Omega) \backslash W(F)$. Thus, $W^{H_{n_{k}}} \stackrel{\text { l.u. }}{\longrightarrow} f_{K_{0}} \circ W \circ g_{H_{0}}=: V$ in $f_{H_{0}}(\Omega \backslash F)$. The domain of $W^{H_{n_{k}}}$ is $\Omega^{H_{n_{k}}}=\widehat{H}_{n_{k}} \cup f_{H_{n_{k}}}\left(\Omega \backslash S_{H_{n_{k}}}\right)$, which converges to $\Omega^{H_{0}}=\widehat{H}_{0} \cup$ $f_{H_{0}}\left(\Omega \backslash S_{H_{0}}\right) \supset f_{H_{0}}(\Omega \backslash F)$. It is clear that $\Omega^{H_{0}} \backslash f_{H_{0}}(\Omega \backslash F)$ is compact. Since $W^{H_{n_{k}}} \stackrel{\text { l.u. }}{\longrightarrow} V$ in $f_{H_{0}}(\Omega \backslash F)$, from the maximum principle, $W^{H_{n_{k}}}$ converges locally uniformly in $\Omega^{H_{0}}$. We still let $V$ denote the limit function. Since $H_{n_{k}} \rightarrow H_{0}$ and $W^{H_{n_{k}}}\left(H_{n_{k}}\right) \rightarrow K_{0}$, we have $V\left(H_{0}\right)=K_{0}$. Since $f_{K_{0}} \circ W \circ g_{H_{0}}=V$ in $f_{H_{0}}(\Omega \backslash F)$, we see that $f_{V\left(H_{0}\right)} \circ W \circ g_{H_{0}}=V$ in $f_{H_{0}}\left(\Omega \backslash S_{H_{0}}\right)$. Thus, $V=W^{H_{0}}$. So $K_{0}=W^{H_{0}}\left(H_{0}\right)=W^{*}\left(H_{0}\right)$. This is the contradiction we need.

(ii). To show $f^{*}$ is continuous, it suffices to show that, if $\left(K_{1}^{n}, K_{2}^{n}\right)$ is a sequence in $\mathcal{H}_{E} \times \mathcal{H}_{F}$ which converges to $\left(K_{1}^{0}, K_{2}^{0}\right) \in \mathcal{H}_{E} \times \mathcal{H}_{F}$, then it has a subsequence $\left(K_{1}^{\left(n_{k}\right)}, K_{2}^{\left(n_{k}\right)}\right)$ such that $f^{*}\left(K_{1}^{\left(n_{k}\right)}, K_{2}^{\left(n_{k}\right)}\right) \rightarrow f^{*}\left(K_{1}^{0}, K_{2}^{0}\right)$. Let $\left(H_{1}^{n}, H_{2}^{n}\right)=f^{*}\left(K_{1}^{n}, K_{2}^{n}\right), n \in \mathbb{N}$. From Theorem 2.16 (iii), $S_{H_{1}^{n} \cup H_{2}^{n}}=S_{K_{1}^{n}} \cup S_{K_{2}^{n}} \subset E \cup F$. From Lemma C.2. $\left(H_{1}^{n} \cup H_{2}^{n}\right)$ has a convergent subsequence with limit in $\mathcal{H}_{E \cup F}$. From Lemma 2.11, $S_{H_{1}^{n}} \subset S_{H_{1}^{n} \cup H_{2}^{n}}^{*} \subset A$, where $A$ is the convex hull of $E \cup F$. From Lemma C.2. $\left(H_{1}^{n}\right)$ has a convergent subsequence. For the same reason, $\left(H_{2}^{n}\right)$ also has a convergent subsequence. By passing to subsequences, we may assume that $H_{1}^{n} \cup H_{2}^{n} \rightarrow M^{0} \in \mathcal{H}_{E \cup F}$ and $H_{j}^{n} \rightarrow H_{j}^{0}, j=1,2$.

From Theorem 2.16 (i) and the continuity of the dot product, we get $H_{1}^{0} \cdot K_{2}^{0}=H_{2}^{0}$. $K_{1}^{0}=M^{0}$. This implies that $M^{0}=H_{1}^{0} \cup f_{H_{1}^{0}}\left(K_{2}^{0}\right)$. The measures $\left(\mu_{H_{1}^{n}}\right)$ (see Appendix C) converges to $\mu_{H_{1}^{0}}$ weakly. Each $\mu_{H_{1}^{n}}$ is supported by $S_{H_{1}^{n}}$. From Theorem 2.16 (ii), $S_{H_{1}^{n}}=f_{K_{2}^{n}}\left(S_{K_{1}^{n}}\right) \subset f_{K_{2}^{n}}(E)$. Since $E$ is a compact subset of $\mathbb{C} \backslash F$, we have $f_{K_{2}^{n}} \rightarrow f_{K_{2}^{0}}$ uniformly on $E$. Thus, $f_{K_{2}^{n}}(E) \rightarrow f_{K_{2}^{0}}(E)$ in the Hausdorff metric. So $\mu_{H_{1}^{0}}$ is supported by $f_{K_{2}^{0}}(E)$, which implies that $S_{H_{1}^{0}} \subset f_{K_{2}^{0}}(E)$. Hence $f_{H_{1}^{0}}\left(K_{2}^{0}\right)$ is another $\mathbb{H}$-hull, which is bounded away from $H_{1}^{0}$. From $K_{2}^{n} \rightarrow K_{2}^{0}$ we have $\mathbb{H} \backslash K_{2}^{n} \stackrel{\text { Cara }}{\longrightarrow} \backslash \mathbb{H} \backslash K_{2}^{0}$. From (C.1) we get $f_{H_{1}^{n}} \stackrel{\text { l.u. }}{\longrightarrow} f_{H_{1}^{0}}$ in $\mathbb{C} \backslash S_{H_{1}^{0}}$. Thus, $\mathbb{H} \backslash f_{H_{1}^{n}}\left(K_{2}^{n}\right) \stackrel{\text { Cara }}{\longrightarrow} \mathbb{H} \backslash f_{H_{1}^{0}}\left(K_{2}^{0}\right)$. Since $H_{2}^{n}=f_{H_{1}^{n}}\left(K_{2}^{n}\right)$, we have $\mathbb{H} \backslash H_{2}^{n} \stackrel{\text { Cara }}{\longrightarrow} \mathbb{H} \backslash f_{H_{1}^{0}}\left(K_{2}^{0}\right)$. On the other hand, $\mathbb{H} \backslash H_{2}^{n} \stackrel{\text { Cara }}{\longrightarrow} \mathbb{H} \backslash H_{2}^{0}$. Since $\mathbb{H} \backslash H_{2}^{0}$ and $\mathbb{H} \backslash f_{H_{1}^{0}}\left(K_{2}^{0}\right)$ both contain a neighborhood of $\infty$ in $\mathbb{H}$, they must be the same domain. Thus, $H_{2}^{0}=f_{H_{1}^{0}}\left(K_{2}^{0}\right)$ is bounded away from $H_{1}^{0}$, i.e., $\left(H_{1}^{n}, H_{2}^{n}\right) \in \mathcal{P}^{*}$. For the same reason, $H_{1}^{0}=f_{H_{2}^{0}}\left(K_{1}^{0}\right)$. Thus, $\left(H_{1}^{n}, H_{2}^{n}\right) \rightarrow\left(H_{1}^{0}, H_{2}^{0}\right)=f^{*}\left(K_{1}^{0}, K_{2}^{0}\right)$. This shows that $f^{*}$ is continuous. Finally, since $K_{1} \vee K_{2}=H_{1} \cdot K_{2}$ if $\left(H_{1}, H_{2}\right)=f^{*}\left(K_{1}, K_{2}\right)$, we see that $\left(K_{1}, K_{2}\right) \mapsto K_{1} \vee K_{2}$ is also continuous.

Corollary 2.19. (i) Let $W$ be an $\mathbb{R}$-symmetric conformal map with domain $\Omega$. Then $W^{*}$ is measurable on $\mathcal{H}_{\Omega \cap \mathbb{R}}$.

(ii) $f^{*}$ and $\left(K_{1}, K_{2}\right) \mapsto K_{1} \vee K_{2}$ are measurable on $\mathcal{P}_{*}$.

Proof. (i) We may find an increasing sequence of compact subsets $\left(F_{n}\right)$ of $\Omega \cap \mathbb{R}$ such that $\mathcal{H}_{\Omega \cap \mathbb{R}}=\bigcup_{n=1}^{\infty} \mathcal{H}_{F_{n}}$. From Theorem 2.18 (i), $W^{*}$ is continuous on each $\mathcal{H}_{F_{n}}$. Thus, $W^{*}$ is measurable on $\mathcal{H}_{\Omega \cap \mathbb{R}}$.

(ii) We may find a sequence of pairs of disjoint bounded closed intervals of $\mathbb{R}:\left(E_{n}, F_{n}\right)$, $n \in \mathbb{N}$, such that $\mathcal{P}_{*}=\bigcup_{n=1}^{\infty} \mathcal{H}_{E_{n}} \times \mathcal{H}_{F_{n}}$. From Theorem 2.18 (ii), $f^{*}$ and $\left(K_{1}, K_{2}\right) \mapsto$ $K_{1} \vee K_{2}$ are continuous on each $\mathcal{H}_{E_{n}} \times \mathcal{H}_{F_{n}}$, and so they are measurable on $\mathcal{P}_{*}$. 


\subsection{Hulls in the unit disc}

A subset $K$ of $\mathbb{D}=\{|z|<1\}$ is called a $\mathbb{D}$-hull if $\mathbb{D} \backslash K$ is a simply connected domain containing 0 . For every $\mathbb{D}$-hull $K$, there is a unique $g_{K}: \mathbb{D} \backslash K \stackrel{\text { Conf }}{\rightarrow} \mathbb{D}$ such that $g_{K}(0)=0$ and $g_{K}^{\prime}(0)>0$. Then $\ln g_{K}^{\prime}(0) \geq 0$ is called the $\mathbb{D}$-capacity of $K$, and is denoted by dcap $(K)$. Let $f_{K}=g_{K}^{-1}$.

We may define $K_{1} \cdot K_{2}, K_{2} / K_{1}$ (when $K_{1} \subset K_{2}$ ), and $K_{1} \prec K_{2}$ on the space of $\mathbb{D}$-hulls as in Definition 2.4. Then the remarks after Definition 2.4 still hold if $\mathbb{H}$ is replaced by $\mathbb{D}$ and hcap is replaced by dcap. Then we may define $K_{2}: K_{1}$ (when $K_{1} \prec K_{2}$ ) as in Definition 2.5. For a $\mathbb{D}$-hull $K$, the base $B_{K}$ of $K$ is $\bar{K} \cap \mathbb{T}$, and the double of $K$ is $\widehat{K}=K \cup I_{\mathbb{T}}(K) \cup B_{K}$, where $I_{\mathbb{T}}(z):=1 / \bar{z}$. Then $g_{K}$ extends to a conformal map (still denoted by $g_{K}$ ) on $\widehat{\mathbb{C}} \backslash \widehat{K}$, which commutes with $I_{\mathbb{T}}$. Moreover, $g_{K}(\widehat{\mathbb{C}} \backslash \widehat{K})=\widehat{\mathbb{C}} \backslash S_{K}$ for some compact $S_{K} \subset \mathbb{T}$, which is called the support of $K$. So $f_{K}$ extends to a conformal map from $\widehat{\mathbb{C}} \backslash S_{K}$ onto $\widehat{\mathbb{C}} \backslash \widehat{K}$, which commutes with $I_{\mathbb{T}}$. Then Lemma 2.6 and Lemma 2.7 still hold here.

We may define $\mathbb{T}$-symmetric sets and $\mathbb{T}$-symmetric conformal maps using Definition 2.8 with $\mathbb{R}$ and $\mathbb{H}$ replaced by $\mathbb{T}$ and $\mathbb{D}$, respectively. For a $\mathbb{T}$-symmetric domain $\Omega$ and a $\mathbb{D}$-hull $K$, we may define domains $\Omega_{K}$ (when $\widehat{K} \subset \Omega$ ) and $\Omega^{K}$ (when $S_{K} \subset \Omega$ ) using Definition 2.9 If $W$ is a $\mathbb{T}$-symmetric conformal map with domain $\Omega$, and if $\Omega_{K}$ is defined, we may then define $W_{K}$ using Definition 2.10 , which is a $\mathbb{T}$-symmetric conformal map on $\Omega_{K}$. The remarks after Definition 2.8, Definition 2.9 and Definition 2.10 hold here with minor modifications. We claim that Theorem 2.12 holds here with modifications. We need several lemmas.

The lemma below relates the $\mathbb{H}$-hulls with $\mathbb{D}$-hulls. To distinguish the two set of symbols, we use $f_{K}^{\mathbb{H}}, g_{K}^{\mathbb{H}}, B_{K}^{\mathbb{R}}, S_{K}^{\mathbb{R}}$, and $\widehat{K}^{\mathbb{R}}$ for $\mathbb{H}$-hulls, and $f_{K}^{\mathbb{D}}, g_{K}^{\mathbb{D}}, B_{K}^{\mathbb{T}}, S_{K}^{\mathbb{T}}$, and $\widehat{K}^{\mathbb{T}}$ for $\mathbb{D}$-hulls.

Theorem 2.20. (i) Let $W$ be a Möbius transformation that maps $\mathbb{D}$ onto $\mathbb{H}$, and $K$ be $a \mathbb{D}$-hull such that $W^{-1}(\infty) \notin S_{K}^{\mathbb{T}}$. Then there is a unique Möbius transformation $W^{K}$ that maps $\mathbb{D}$ onto $\mathbb{H}$ such that $W^{K}(K)$ is an $\mathbb{H}-$-hull, $g_{W^{K}(K)}^{\mathbb{H}} \circ W^{K} \circ f_{K}^{\mathbb{D}}=W$ in $\widehat{\mathbb{C}} \backslash S_{K}^{\mathbb{T}}$, and $S_{W^{K}(K)}^{\mathbb{R}}=W\left(S_{K}^{\mathbb{T}}\right)$.

(ii) Let $W$ be a Möbius transformation that maps $\mathbb{H}$ onto $\mathbb{D}$, and $K$ be an $\mathbb{H}-$-hull. Then there is a unique Möbius transformation $W^{K}$ that maps $\mathbb{H}$ onto $\mathbb{D}$ such that $W^{K}(K)$ is a $\mathbb{D}$-hull, $g_{W^{K}(K)}^{\mathbb{D}} \circ W^{K} \circ f_{K}^{\mathbb{H}}=W$ in $\widehat{\mathbb{C}} \backslash S_{K}^{\mathbb{R}}$, and $S_{W^{K}(K)}^{\mathbb{T}}=W\left(S_{K}^{\mathbb{R}}\right)$.

Proof. (i) Let $z_{0}=W^{-1}(\infty) \in \mathbb{T} \backslash S_{K}^{\mathbb{T}}$. Then $w_{0}:=f_{K}^{\mathbb{D}}\left(z_{0}\right) \in \mathbb{T} \backslash B_{K}^{\mathbb{T}}$ is well defined. Let $W_{0}^{K}(z)=i \frac{w_{0}+z}{w_{0}-z}$. Then $W_{0}^{K}$ is a Möbius transformation that maps $\mathbb{D}$ onto $\mathbb{H}$ and takes $w_{0}$ to $\infty$. Let $L_{0}=W_{0}^{K}(K)$. Since $w_{0}$ is bounded away from $K$, we see that $L_{0}$ is an $\mathbb{H}$-hull. We have $W_{0}^{K}: \widehat{\mathbb{C}} \backslash \widehat{K} \stackrel{\text { Conf }}{\rightarrow} \widehat{\mathbb{C}} \backslash \widehat{L}_{0}$. Define $G=g_{L_{0}}^{\mathbb{H}} \circ W_{0}^{K} \circ f_{K}^{\mathbb{D}} \circ W^{-1}$ on $\widehat{\mathbb{C}} \backslash W\left(S_{K}^{\mathbb{T}}\right)$. Then $G: \widehat{\mathbb{C}} \backslash W\left(S_{K}^{\mathbb{T}}\right) \stackrel{\text { Conf }}{\rightarrow} \widehat{\mathbb{C}} \backslash S_{L_{0}}^{\mathbb{R}}$, fixes $\infty$, and maps $\mathbb{H}$ onto $\mathbb{H}$. So $G(z)=a z+b$ for some $a>0$ and $b \in \mathbb{R}$. Let $W^{K}=G^{-1} \circ W_{0}^{K}$. Then $W^{K}$ is also a Möbius transformation that maps $\mathbb{D}$ onto $\mathbb{H}$, and $W^{K}(K)$ is also an $\mathbb{H}$-hull with $S_{W^{K}(K)}^{\mathbb{R}}=G^{-1}\left(S_{L_{0}}^{\mathbb{R}}\right)=W\left(S_{K}^{\mathbb{T}}\right)$ and $g_{W^{K}(K)}^{\mathbb{H}}=G^{-1} \circ g_{L_{0}}^{\mathbb{H}} \circ G$. Thus,

$$
\begin{gathered}
g_{W^{K}(K)}^{\mathbb{H}} \circ W^{K} \circ f_{K}^{\mathbb{D}} \circ W^{-1}=G^{-1} \circ g_{L_{0}}^{\mathbb{H}} \circ G \circ G^{-1} \circ W_{0}^{K} \circ f_{K}^{\mathbb{D}} \circ W^{-1} \\
=G^{-1} \circ g_{L_{0}}^{\mathbb{H}} \circ W_{0}^{K} \circ f_{K}^{\mathbb{D}} \circ W^{-1}=G^{-1} \circ G=\operatorname{id}_{\widehat{\mathbb{C}} \backslash W\left(S_{K}\right)} .
\end{gathered}
$$


This implies that $g_{L}^{\mathbb{H}} \circ W^{K} \circ f_{K}^{\mathbb{D}}=W$ in $\widehat{\mathbb{C}} \backslash S_{K}$. So we proved the existence. On the other hand, if $W^{K}$ satisfies the desired property, then from $W^{K}=f_{L}^{\mathbb{H}} \circ W \circ g_{K}^{\mathbb{D}}$ we get $W^{K}\left(w_{0}\right)=\infty$. So $W^{K}=G_{0} \circ W_{0}^{K}$, where $G_{0}(z)=a z+b$ for some $a>0$ and $b \in \mathbb{R}$. The above argument shows that $G_{0}=G^{-1}$. So we get the uniqueness.

(ii) We may use the proof of (i) with slight modifications: replace $\infty$ by 0 , swap $\mathbb{H}$ and $\mathbb{D}$, swap $\mathbb{R}$ and $\mathbb{T}$, and define $W_{0}^{K}(z)=\frac{z-w_{0}}{z-\overline{w_{0}}}$.

We also use $W^{*}(K)$ to denote the hull $W^{K}(K)$ in the above lemma. The following lemma is similar to Lemma 2.14.

Lemma 2.21. Let $K_{1}$ and $K_{2}$ be two $\mathbb{H}$-(resp. $\mathbb{D}$-)hulls such that $K_{1} \prec K_{2}$. Let $W$ be a Möbius transformation that maps $\mathbb{H}$ onto $\mathbb{D}$ (resp. maps $\mathbb{D}$ onto $\mathbb{H}$ ) such that $\infty \notin W\left(S_{K_{2}}\right)$. Then $W^{*}\left(K_{1}\right) \prec W^{*}\left(K_{2}\right)$ and (2.2) still holds.

The following lemma is used to treat the case $S_{K}=\mathbb{T}$ in Theorem 2.23 .

Lemma 2.22. Let $W$ be a $\mathbb{T}$-symmetric conformal map with domain $\Omega \supset \mathbb{T}$. Let $\left(K_{n}\right)$ be a sequence of $\mathbb{D}$-hulls which converges to $K$. Suppose that for each $n$, there is a $\mathbb{T}$-symmetric conformal map $V^{\langle n\rangle}$ defined on $\Omega^{K_{n}}$ such that $V_{K_{n}}^{\langle n\rangle}=W$. Then there is a $\mathbb{T}$-symmetric conformal map $V$ defined on $\Omega^{K}$ such that $V_{K}=W$. Moreover, $V(K)$ is a subsequential limit of $\left(V^{\langle n\rangle}\left(K_{n}\right)\right)$.

Proof. Since $K_{n} \rightarrow K, \Omega^{K_{n}} \stackrel{\text { Cara }}{\longrightarrow} \Omega^{K}$. Since $V^{\langle n\rangle}$ maps $\Omega^{K_{n}} \cap \mathbb{D}$ into $\mathbb{D}$, the family $\left(\left.V^{\langle n\rangle}\right|_{\Omega^{K_{n}} \cap \mathbb{D}}\right)$ is uniformly bounded. Thus, $\left(V^{\langle n\rangle}\right)$ contains a subsequence, which convergence locally uniformly in $\Omega^{K} \cap \mathbb{D}$. To save the symbols, we assume that $\left(V^{\langle n\rangle}\right)$ itself converges locally uniformly in $\Omega^{K} \cap \mathbb{D}$. Since each $V^{\langle n\rangle}$ is $\mathbb{T}$-symmetric, the sequence also converges locally uniformly in $\Omega^{K} \cap \mathbb{D}^{*}$. From the maximum principle, $\left(V^{\langle n\rangle}\right)$ converges locally uniformly in $\Omega^{K}$. Let $V$ be the limit function. Since each $V^{\langle n\rangle}$ maps $\mathbb{T}$ onto $\mathbb{T}$, and $V^{\langle n\rangle} \rightarrow V$ uniformly on $\mathbb{T}, V$ can not be constant. From Lemma A.2 $V$ is a conformal map. It is $\mathbb{T}$-symmetric because each $V^{\langle n\rangle}$ is $\mathbb{T}$-symetric. Since $K_{n} \rightarrow K$, we have $V^{\langle n\rangle}\left(K_{n}\right) \rightarrow V(K)$. From $V_{K_{n}}^{\langle n\rangle}=W$ we have $g_{V^{\langle n\rangle}\left(K_{n}\right)} \circ V^{\langle n\rangle} \circ f_{K_{n}}=W$ in $\Omega \backslash \mathbb{T}$. Letting $n \rightarrow \infty$ we get $g_{V(K)} \circ V \circ f_{K}=W$ in $\Omega \backslash \mathbb{T}$. By continuation, this equality also holds on $\Omega \backslash S_{K}$. Thus, $V_{K}=W$.

Theorem 2.23. Let $W$ be a $\mathbb{T}$-symmetric conformal map with domain $\Omega$. Let $K$ be a $\mathbb{D}$-hull such that $S_{K} \subset \Omega$. Then there is a unique $\mathbb{T}$-symmetric conformal map $V$ defined on $\Omega^{K}$ such that $V_{K}=W$.

Proof. We first consider the existence. Case 1. $S_{K}^{\mathbb{T}} \neq \mathbb{T}$. We will apply Theorems 2.12 and 2.20 for this case. Pick $z_{0} \in \mathbb{T} \backslash S_{K}^{\mathbb{T}}$ and let $h(z)=i \frac{z_{0}+z}{z_{0}-z}$. From Theorem 2.20 (i), there is a Möbius transformation $h^{K}$ that maps $\mathbb{D}$ onto $\mathbb{H}$ such that $L:=h^{K}(K)$ is an $\mathbb{H}$-hull, and $g_{L}^{\mathbb{H}} \circ h^{K} \circ f_{K}^{\mathbb{D}}=h$ in $\widehat{\mathbb{C}} \backslash S_{K}^{\mathbb{T}}$. Since $W$ is a homeomorphism on $S_{K}, W\left(S_{K}\right) \neq \mathbb{T}$. So there is $z_{W} \in \mathbb{T} \backslash W\left(S_{K}\right)$. Let $h_{W}(z)=z_{W} \cdot \frac{z-i}{z+i}$. Then $h_{W}$ is a Möbius transformation that maps $\mathbb{H}$ onto $\mathbb{D}$ and takes $\infty$ to $z_{W}$. Let $\widetilde{W}=h_{W}^{-1} \circ W \circ h^{-1}$. Then $\widetilde{W}$ is an $\mathbb{R}$ symmetric conformal map with domain $h(\Omega)$, and $\widetilde{W}\left(S_{L}^{\mathbb{R}}\right)=h_{W}^{-1} \circ W\left(S_{K}^{\mathbb{T}}\right) \not \supset \infty$. From Theorem 2.12, there is an $\mathbb{R}$-symmetric conformal map $\widetilde{V}$ with domain $\widehat{L}^{\mathbb{R}} \cup f_{L}^{\mathbb{R}}\left(h(\Omega) \backslash S_{L}^{\mathbb{R}}\right)$ such that $L^{*}:=\widetilde{V}(L)$ is an $\mathbb{H}$-hull, and $\widetilde{V}=f_{L^{*}}^{\mathbb{H}} \circ \widetilde{W} \circ g_{L}^{\mathbb{H}}$ in $\widehat{\mathbb{C}} \backslash \widehat{L}^{\mathbb{R}}$. From Theorem 2.20 
(ii), there is a Möbius transformation $h_{W}^{L^{*}}$ that maps $\mathbb{H}$ onto $\mathbb{D}$ such that $K^{*}:=h_{W}^{L^{*}}\left(L^{*}\right)$ is a $\mathbb{D}$-hull, and $g_{K^{*}}^{\mathbb{D}} \circ h_{W}^{L^{*}} \circ f_{L^{*}}^{\mathbb{H}}=h_{W}$ in $\widehat{\mathbb{C}} \backslash S_{L^{*}}^{\mathbb{R}}$. Finally, let $V=h_{W}^{L^{*}} \circ \widetilde{V} \circ h^{K}$. Then

$$
V(K)=h_{W}^{L^{*}} \circ \widetilde{V}(L)=h_{W}^{L^{*}}\left(L^{*}\right)=K^{*},
$$

and

$$
\begin{gathered}
g_{K^{*}} \circ V \circ f_{K}=g_{K^{*}} \circ h_{W}^{L^{*}} \circ \widetilde{V} \circ h^{K} \circ f_{K} \\
=g_{K^{*}} \circ h_{W}^{L^{*}} \circ f_{L^{*}}^{\mathbb{H}} \circ \widetilde{W} \circ g_{L}^{\mathbb{H}} \circ h^{K} \circ f_{K} \\
=h_{W} \circ \widetilde{W} \circ h=W
\end{gathered}
$$

in $\widehat{\mathbb{C}} \backslash \widehat{K}$. This finishes the existence part for Case 1 .

Case 2. $S_{K}=\mathbb{T}$. First, we may approximate $K$ using $\mathbb{D}$-hulls bounded by $\mathbb{T}$ and a Jordan curve in $\mathbb{D}$. For example, let $J_{n}=f_{K}(\{|z|=1-1 /(2 n)\})$, and let $K_{n}=\mathbb{D} \backslash D_{J_{n}}$. Then each $K_{n}$ is a $\mathbb{D}$-hull, and $K_{n} \rightarrow K$. Second, if $K^{\prime}$ has the form of $\mathbb{D} \backslash D_{J}$ for some Jordan curve $J$, then we may define a curve $\beta$, which starts from $\beta(0)=z_{0} \in \mathbb{T}$, then follows a simple curve in $\mathbb{D} \cap D_{J}^{*}$ to a point on $J$, and then follows $J$ in the clockwise direction, and ends when it finishes one round. Suppose the domain of $\beta$ is $[0,1]$. Then $\beta$ is simple on $[0,1-\varepsilon]$ for any $\varepsilon>0$. Let $K_{n}=\beta((0,1-1 / n]), n \in \mathbb{N}$. Then each $K_{n}$ is a $\mathbb{D}$-hull with $S_{K_{n}} \neq \mathbb{T}$, and $K_{n} \rightarrow K^{\prime}$. Thus, $K$ can be approximated by a sequence of $\mathbb{D}$-hulls $\left(K_{n}\right)$ such that $S_{K_{n}} \neq \mathbb{T}$ for each $K_{n}$. Then the existence of $V$ follows from Case 1 and Lemma 2.22

Now we prove the uniqueness. Suppose $\widetilde{V}$ is another $\mathbb{T}$-symmetric conformal map defined on $\Omega^{K}$ such that $\widetilde{V}_{K}=W$. We may use the argument in the proof of Theorem 2.12 to construct an analytic function $h$ on $\mathbb{C}$ such that $h=\widetilde{V} \circ V^{-1}$ on $V(\Omega)$ and $h=f_{\widetilde{V}(K)} \circ g_{V(K)}$ on $\mathbb{C} \backslash V(\widehat{K})$. Then $h$ is $\mathbb{T}$-symmetric. From the properties of $f_{\widetilde{V}(K)}$ and $g_{V(K)}$, we see that $h(0)=0$ and $h^{\prime}(0)>0$. So $h=$ id, which implies that $\widetilde{V}=V$.

We may then define $W^{K}$ and $W^{*}$ using Definition 2.13 with Theorem 2.23 in place of Theorem 2.12 and $\mathbb{D}$ in place of $\mathbb{H}$. The remarks after Definition 2.13 hold here with minor modifications, and so does Lemma 2.14. Then we define $\mathcal{P}^{*}, \mathcal{P}_{*}, g_{*}$, and $f^{*}$ using Definition 2.15 with $\mathbb{H}$ replaced by $\mathbb{D}$. Then Theorem 2.16 still holds here, and we may define the quotient union $K_{1} \vee K_{2}$ for $\left(K_{1}, K_{2}\right) \in \mathcal{P}_{*}$.

The space of $\mathbb{D}$-hulls has a natural metric $d_{\mathcal{H}}$ described in Appendix $\mathrm{D}$. Let $\mathcal{H}_{S}$ denote the set of $\mathbb{D}$-hulls whose supports are contained in $S$. We claim that Theorem 2.18 still holds here if every $\mathbb{R}$ is replaced by $\mathbb{T}$. For part (i), if $F \neq \mathbb{T}$, then the proof of Theorem 2.18 (i) still goes through with Lemma D.2 in place of Lemma C.2 if $F=\mathbb{T}$, then the continuity of $W^{*}$ follows from Lemma 2.22, For part (ii), the proof of Theorem 2.18 still goes through with some modifications. The relatively compactness of $\left(H_{n} \cup J_{n}\right)$ follows from Lemma D.2 instead of Lemma C.2 because $S_{H_{n} \cup J_{n}} \subset E \cup F \subsetneq \mathbb{T}$. To show the relatively compactness of $\left(H_{n}\right)$ and $\left(J_{n}\right)$, instead of applying Lemma 2.11, we now apply Lemma D.1, and use the relatively compactness of $\left(H_{n} \cup J_{n}\right)$ and the inequalities $\operatorname{dcap}\left(H_{n}\right)$, $\operatorname{dcap}\left(J_{n}\right) \leq \operatorname{dcap}\left(H_{n} \cup J_{n}\right)$. In addition, (D.2) will be used in place of (C.1). This finishes the proof of Theorem 2.18 in the radial case. Then Corollary 2.19 in the radial case immediately follows.

The proof of Theorem 2.18 (i) may also be used to show that the map $K \mapsto W^{K}(K)$ in Theorem 2.20 (i) (resp. (ii)) is continuous if restricted to $\mathcal{H}_{F}^{\mathbb{D}}$ (resp. $\mathcal{H}_{F}^{\mathbb{H}}$ ), where $F$ is a compact subset of $\mathbb{T} \backslash W^{-1}(\infty)$ (resp. $\left.\mathbb{R}\right)$. We then can conclude that the maps $K \mapsto W^{K}(K)$ in Theorem 2.20 (i) and (ii) are both measurable. 


\section{Loewner Equations and Loewner Chains}

\subsection{Forward Loewner equations}

We review the definitions and basic facts about (forward) Loewner equations. The reader is referred to [6] for details. Let $\lambda \in C([0, T))$, where $T \in(0, \infty]$. The chordal Loewner equation driven by $\lambda$ is

$$
\partial_{t} g_{t}(z)=\frac{2}{g_{t}(z)-\lambda(t)}, \quad g_{0}(z)=z .
$$

We assume that $g_{t}(\infty)=\infty$ for $0 \leq t<\infty$. For $z \in \mathbb{C}$, suppose that the maximal interval for $t \mapsto g_{t}(z)$ is $\left[0, \tau_{z}\right)$. Let $K_{t}=\left\{z \in \mathbb{H}: \tau_{z} \leq t\right\}$, i.e., the set of $z \in \mathbb{H}$ such that $g_{t}(z)$ is not defined. Then $g_{t}$ and $K_{t}, 0 \leq t<T$, are called the chordal Loewner maps and hulls driven by $\lambda$. It is known that each $K_{t}$ is an $\mathbb{H}$-hull with hcap $\left(K_{t}\right)=2 t$, and for $0<t<T$, $g_{t}=g_{K_{t}}$ with exactly the same domain: $\widehat{\mathbb{C}} \backslash \widehat{K}_{t}$. At $t=0, K_{0}=\emptyset$ and $g_{0}=\operatorname{id} \widehat{\widehat{\mathbb{C}} \backslash\{\lambda(0)\}}$.

We say that $\lambda$ generates a chordal trace $\beta$ if

$$
\beta(t):=\lim _{\mathbb{H} \ni z \rightarrow \lambda(t)} g_{t}^{-1}(z) \in \overline{\mathbb{H}}
$$

exists for $0 \leq t<T$, and $\beta$ is a continuous curve. We call such $\beta$ the chordal trace driven by $\lambda$. If the chordal trace $\beta$ exists, then for each $t, \mathbb{H} \backslash K_{t}$ is the unbounded component of $\mathbb{H} \backslash \beta((0, t])$, and $f_{t}$ extends continuously from $\mathbb{H}$ to $\mathbb{H} \cup \mathbb{R}$. The trace $\beta$ is called simple if it is a simple curve and $\beta(t) \in \mathbb{H}$ for $0<t<T$, in which case $K_{t}=\beta((0, t])$ for $0 \leq t<T$.

The radial Loewner equation driven by $\lambda$ is

$$
\partial_{t} g_{t}(z)=g_{t}(z) \frac{e^{i \lambda(t)}+g_{t}(z)}{e^{i \lambda(t)}-g_{t}(z)}, \quad 0 \leq t<T ; \quad g_{0}(z)=z .
$$

We assume that $g_{t}(\infty)=\infty$ for $0 \leq t<\infty$. For each $t \in[0, T)$, let $K_{t}$ be the set of $z \in \mathbb{D}:=\{|z|<1\}$ at which $g_{t}$ is not defined. Then $g_{t}$ and $K_{t}, 0 \leq t<T$, are called the radial Loewner maps and hulls driven by $\lambda$. It is known that, each $K_{t}$ is a $\mathbb{D}$-hull with $\operatorname{dcap}\left(K_{t}\right)=t$, and for $0<t<T, g_{t}=g_{K_{t}}$ with exactly the same domain: $\widehat{\mathbb{C}} \backslash \widehat{K}_{t}$. At $t=0$, $K_{0}=\emptyset$ and $g_{0}=\operatorname{id}_{\widehat{\mathbb{C}} \backslash\left\{e^{i \lambda(0)}\right\}}$.

We say that $\lambda$ generates a radial trace $\beta$ if

$$
\beta(t):=\lim _{\mathbb{D} \ni z \rightarrow e^{i \lambda(t)}} g_{t}^{-1}(z) \in \overline{\mathbb{D}}
$$

exists for $0 \leq t<T$, and $\beta$ is a continuous curve. We call such $\beta$ the radial trace driven by $\lambda$. If the radial trace $\beta$ exists, then for each $t, \mathbb{D} \backslash K_{t}$ is the component of $\mathbb{D} \backslash \beta((0, t])$ that contains 0 . The trace $\beta$ is called simple if it is a simple curve and $\beta(t) \in \mathbb{D}$ for $0<t<T$, in which case $K_{t}=\beta((0, t])$ for $0 \leq t<T$.

Let $\cot _{2}(z)=\cot (z / 2)$. The covering radial Loewner equation driven by $\lambda$ is

$$
\partial \widetilde{g}_{t}(z)=\cot _{2}\left(\widetilde{g}_{t}(z)-\lambda(t)\right), \quad 0 \leq t<T, \quad \widetilde{g}_{0}(z)=z .
$$

For each $t \in[0, T)$, let $\widetilde{K}_{t}$ be the set of all $z \in \mathbb{H}$ at which $\widetilde{g}_{t}$ is not defined. Then $\widetilde{g}_{t}$ and $\widetilde{K}_{t}, 0 \leq t<T$, are called the covering radial Loewner maps and hulls driven by $\lambda$. We have $\widetilde{g}_{t}: \mathbb{H} \backslash \widetilde{K}_{t} \stackrel{\text { Conf }}{\rightarrow} \mathbb{H}$. If $g_{t}$ and $K_{t}, 0 \leq t<T$, are the radial Loewner maps and hulls driven by $\lambda$, then $\widetilde{K}_{t}=\left(e^{i}\right)^{-1}\left(K_{t}\right)$ and $e^{i} \circ \widetilde{g}_{t}=g_{t} \circ e^{i}$, where $e^{i}$ denotes the map $z \mapsto e^{i z}$. 
For $\kappa>0$, chordal (resp. radial) $\mathrm{SLE}_{\kappa}$ is defined by solving the chordal (resp. radial) Loewner equation with $\lambda(t)=\sqrt{\kappa} B(t)$. Such driving function a.s. generates a chordal (resp. radial) trace, which is simple if $\kappa \in(0,4]$.

\subsection{Backward Loewner equations}

Let $\lambda \in C([0, T))$. The backward chordal Loewner equation driven by $\lambda$ is

$$
\partial_{t} f_{t}(z)=\frac{-2}{f_{t}(z)-\lambda(t)}, \quad f_{0}(z)=z .
$$

We assume that $f_{t}(\infty)=\infty$ for $0 \leq t<T$. Let $L_{t}=\mathbb{H} \backslash f_{t}(\mathbb{H})$. We call $f_{t}$ and $L_{t}$, $0 \leq t<T$, the backward chordal Loewner maps and hulls driven by $\lambda$.

Define a family of maps $f_{t_{2}, t_{1}}, t_{1}, t_{2} \in[0, T)$, such that, for any fixed $t_{1} \in[0, T)$ and $z \in \widehat{\mathbb{C}} \backslash\left\{\lambda\left(t_{1}\right)\right\}$, the function $t_{2} \mapsto f_{t_{2}, t_{1}}(z)$ is the maximal solution of the ODE

$$
\partial_{t_{2}} f_{t_{2}, t_{1}}(z)=\frac{-2}{f_{t_{2}, t_{1}}(z)-\lambda\left(t_{2}\right)}, \quad f_{t_{1}, t_{1}}(z)=z .
$$

Note that $f_{t, 0}=f_{t}$ and $f_{t, t}=\operatorname{id}_{\widehat{\mathbb{C}} \backslash\{\lambda(t)\}}, 0 \leq t<T$. If $t_{1} \in(0, T)$, then $t_{2}$ could be bigger or smaller than $t_{1}$. Some simple observations give the following lemma.

Lemma 3.1. (i) For any $t_{1}, t_{2}, t_{3} \in[0, T), f_{t_{3}, t_{2}} \circ f_{t_{2}, t_{1}}$ is a restriction of $f_{t_{3}, t_{1}}$. In particular, this implies that $f_{t_{1}, t_{2}}=f_{t_{2}, t_{1}}^{-1}$.

(ii) For any fixed $t_{0} \in[0, T), f_{t_{0}+t, t_{0}}, 0 \leq t<T-t_{0}$, are the backward chordal Loewner maps driven by $\lambda\left(t_{0}+t\right), 0 \leq t<T-t_{0}$.

(iii) For any fixed $t_{0} \in[0, T), f_{t_{0}-t, t_{0}}, 0 \leq t \leq t_{0}$, are the (forward) chordal Loewner maps driven by $\lambda\left(t_{0}-t\right), 0 \leq t \leq t_{0}$.

Let $L_{t_{2}, t_{1}}=\mathbb{H} \backslash f_{t_{2}, t_{1}}(\mathbb{H})$ for $0 \leq t_{1} \leq t_{2}<T$. From (i), (iii), and the properties of forward chordal Loewner maps, we see that, if $0 \leq t_{1}<t_{2}<T$, then $L_{t_{2}, t_{1}}$ is an $\mathbb{H}$-hull with hcap $\left(L_{t_{2}, t_{1}}\right)=2\left(t_{2}-t_{1}\right)$, and $f_{t_{2}, t_{1}}=f_{L_{t_{2}, t_{1}}}$. If $t_{1}=t_{2}$, this is almost still true except that $f_{t_{1}, t_{1}}=\operatorname{id}_{\widehat{\mathbb{C}} \backslash\left\{\lambda\left(t_{1}\right)\right\}}$ and $f_{L_{t_{1}, t_{1}}}=f_{\emptyset}=\operatorname{id}_{\widehat{\mathbb{C}}}$. Since $L_{t, 0}=L_{t}$, and $\lambda(t) \in \mathbb{R}$ does not lie in the range of $f_{t}$, which is $\widehat{\mathbb{C}} \backslash \widehat{L}_{t}$ for $t>0$, we get the following lemma.

Lemma 3.2. For $0 \leq t<T, L_{t}$ is an $\mathbb{H}$-hull with $\mathrm{hcap}\left(L_{t}\right)=2 t$. If $t \in(0, T)$, then $f_{t}=f_{L_{t}}$ with the same domain: $\widehat{\mathbb{C}} \backslash S_{L_{t}}$, and $\lambda(t) \in B_{L_{t}}$.

If $t_{2} \geq t_{1} \geq t_{0}$, from $f_{t_{2}, t_{1}} \circ f_{t_{1}, t_{0}}=f_{t_{2}, t_{0}}$ we get $L_{t_{2}, t_{0}}=L_{t_{2}, t_{1}} \cdot L_{t_{1}, t_{0}}$. From Lemmas 2.7 and 3.1 , we obtain the following lemma.

Lemma 3.3. For any $0 \leq t_{1}<t_{2}<T, L_{t_{1}} \prec L_{t_{2}}$ and $S_{L_{t_{1}}} \subset S_{L_{2}\left(t_{2}\right)}$. For any fixed $t_{0} \in[0, T)$, the family $L_{t_{0}}: L_{t_{0}-t}=L_{t_{0}, t_{0}-t}, 0 \leq t \leq t_{0}$, are the chordal Loewner hulls driven by $\lambda\left(t_{0}-t\right), 0 \leq t \leq t_{0}$.

Note that $S_{L_{0}}=S_{\emptyset}=\emptyset$, and its is easy to see that, for $0<t_{0}<T, S_{L_{t_{0}}}$ is the set of $x \in \mathbb{R}$ such that the solution $f_{t}(x)$ to 3.1 blows up before or at $t_{0}$, i.e., $S_{L_{t_{0}}}=\left\{x \in \mathbb{R}: \tau_{x} \leq t_{0}\right\}$. So every $S_{L_{t}}, 0<t<T$, is a real interval, and $\bigcap_{0<t<T} S_{L_{t}}=\{\lambda(0)\}$.

If for every $t_{0} \in[0, T), \lambda\left(t_{0}-t\right), 0 \leq t \leq t_{0}$, generates a (forward) chordal trace, which we denote by $\beta_{t_{0}}\left(t_{0}-t\right), 0 \leq t \leq t_{0}$, then we say that $\lambda$ generates backward chordal traces 
$\beta_{t_{0}}, 0 \leq t_{0}<T$. If this happens, then for any $0 \leq t_{1} \leq t_{2}<T, \mathbb{H} \backslash L_{t_{2}, t_{1}}$ is the unbounded component of $\mathbb{H} \backslash \beta_{t_{2}}\left(\left[t_{1}, t_{2}\right)\right)$, and $f_{t_{2}, t_{1}}$ extends continuously from $\mathbb{H}$ to $\overline{\mathbb{H}}$ such that

$$
f_{t_{2}, t_{1}}\left(\lambda\left(t_{1}\right)\right)=\beta_{t_{2}}\left(t_{1}\right), \quad 0 \leq t_{1} \leq t_{2}<T .
$$

Here we still use $f_{t_{2}, t_{1}}$ to denote the continuation if there is no confusion. For $0 \leq t_{0} \leq t_{1} \leq$ $t_{2}<T$, the equality $f_{t_{2}, t_{0}}=f_{t_{2}, t_{1}} \circ f_{t_{1}, t_{0}}$ still holds after continuation, which together with (3.2) implies that

$$
f_{t_{2}, t_{1}}\left(\beta_{t_{1}}(t)\right)=\beta_{t_{2}}(t), \quad 0 \leq t \leq t_{1} \leq t_{2}<T .
$$

Remark. One should keep in mind that each $\beta_{t}$ is a continuous function defined on $[0, t]$, $\beta_{t}(0)$ is the tip of $\beta_{t}$, and $\beta_{t}(t)$ is the root of $\beta_{t}$, which lies on $\mathbb{R}$. The parametrization is different from a forward chordal trace $\beta$, of which $\beta(0)$ is the root.

The backward radial Loewner equations and the backward covering radial Loewner equation driven by $\lambda \in C([0, T))$ are the following two equations respectively:

$$
\begin{array}{ll}
\partial_{t} f_{t}(z)=-f_{t}(z) \frac{e^{i \lambda(t)}+f_{t}(z)}{e^{i \lambda(t)}-f_{t}(z)}, & f_{0}(z)=z ; \\
\partial_{t} \widetilde{f}_{t}(z)=-\cot _{2}\left(\widetilde{f}_{t}(z)-\lambda(t)\right), \quad \widetilde{f}_{0}(z)=z .
\end{array}
$$

We have $f_{t} \circ e^{i}=e^{i} \circ \widetilde{f}_{t}$. Let $L_{t}=\mathbb{D} \backslash f_{t}(\mathbb{D})$. We call $f_{t}$ and $L_{t}, 0 \leq t<T$, the backward radial Loewner maps and hulls driven by $\lambda$, and call $\widetilde{f}_{t}, 0 \leq t<T$, the backward covering radial Loewner maps driven by $\lambda$.

By introducing $f_{t_{2}, t_{1}}$ in the radial setting, we find that Lemma 3.1 holds if the word "chordal" is replaced by "radial". The following lemma is similar to Lemma 3.2

Lemma 3.4. For $0 \leq t<T, L_{t}$ is a $\mathbb{D}$-hull with dcap $\left(L_{t}\right)=t$. If $t \in(0, T)$, then $f_{t}=f_{L_{t}}$ with the same domain: $\widehat{\mathbb{C}} \backslash S_{L_{t}}$, and $e^{i \lambda(t)} \in B_{L_{t}}$.

We find that Lemma 3.3 holds here if the word "chordal" is replaced by "radial". So we may define backward radial traces $\beta_{t}, 0 \leq t<T$, in a similar manner.

The following lemma holds only in the radial case.

Lemma 3.5. If $T=\infty$, then $\mathbb{T} \backslash \bigcup_{0<t<\infty} S_{L_{t}}$ contains at most one point.

Proof. Let $S_{\infty}=\bigcup_{0<t<\infty} S_{L_{t}}$. From Koebe's $1 / 4$ theorem, as $t \rightarrow \infty$, $\operatorname{dist}\left(0, L_{t}\right) \rightarrow 0$, which implies that the harmonic measure of $\mathbb{T} \backslash B_{L_{t}}$ in $\mathbb{D} \backslash L_{t}$ seen from 0 tends to 0 . Since $f_{t}: \mathbb{D} \stackrel{\text { Conf }}{\rightarrow} \mathbb{D} \backslash L_{t}, f_{t}(0)=0$, and $f_{t}\left(\mathbb{T} \backslash S_{L_{t}}\right)=\mathbb{T} \backslash B_{L_{t}}$, the above harmonic measure at time $t$ equals to $\left|\mathbb{T} \backslash S_{L_{t}}\right| /(2 \pi)$. Thus, $\left|\mathbb{T} \backslash S_{\infty}\right|=\lim _{t \rightarrow \infty}\left|\mathbb{T} \backslash S_{L_{t}}\right|=0$.

For $\kappa>0$, the backward chordal (resp. radial) $\mathrm{SLE}_{\kappa}$ is defined by solving backward chordal (resp. radial) Loewner equation with $\lambda(t)=\sqrt{\kappa} B(t), 0 \leq t<\infty$. Since for any fixed $t_{0}>0,\left(\lambda\left(t_{0}-t\right)-\lambda\left(t_{0}\right), 0 \leq t \leq t_{0}\right)$ has the distribution of $\left(\sqrt{\kappa} B(t), 0 \leq t \leq t_{0}\right)$, using the existence of forward chordal (resp. radial) $\mathrm{SLE}_{\kappa}$ traces, we conclude that $\lambda$ a.s. generates a family of backward chordal (resp. radial) traces. 


\subsection{Normalized global backward trace}

First we consider a backward chordal Loewner process generated by $\lambda(t), 0 \leq t<T$. Let $S_{t}=S_{L_{t}}, 0 \leq t<T$, and $S_{T}=\bigcup_{0 \leq t<T} S_{t}$. Then $\left(S_{t}\right)$ is an increasing family, and $S_{T}$ is an interval. The following Lemma is similar in spirit to Proposition 5.1 in [16].

Lemma 3.6. There exists a family of conformal maps $F_{T, t}, 0 \leq t<T$, on $\mathbb{H}$ such that $F_{T, t_{1}}=F_{T, t_{2}} \circ f_{t_{2}, t_{1}}$ in $\mathbb{H}$ if $0 \leq t_{1} \leq t_{2}<T$. Let $D_{t}=F_{T, t}(\mathbb{H}), 0 \leq t<T$, and $D_{T}=\bigcup_{t<T} D_{t}$. If $\left(\widehat{F}_{T, t}\right)$ satisfies the same property as $\left(F_{T, t}\right)$, then there is a conformal map $h_{T}$ defined on $D_{T}$ such that $\widehat{F}_{T, t}=h_{T} \circ F_{T, t}, 0 \leq t<T$. If there is $z_{0} \in \mathbb{H}$ such that

$$
\lim _{t \rightarrow T} \frac{\operatorname{Im} f_{t}\left(z_{0}\right)}{\left|f_{t}^{\prime}\left(z_{0}\right)\right|}=\infty
$$

then we may construct $\left(F_{T, t}\right)$ such that $D_{T}=\mathbb{C}$, and we have $S_{T}=\mathbb{R}$.

Proof. Fix $z_{0} \in \mathbb{H}$. Let $z_{t}=f_{t}\left(z_{0}\right)$ and $u_{t}=f_{t}^{\prime}\left(z_{0}\right), 0 \leq t<T$. For $t \in[0, T)$, let $M_{t}(z)=\frac{z-z_{t}}{u_{t}}$ and $F_{t}=M_{t} \circ f_{t}$. Then $F_{t}$ maps $z_{0}$ to 0 and has derivative 1 at $z_{0}$. For $0 \leq t_{1} \leq t_{2}<T$, define $F_{t_{2}, t_{1}}=M_{t_{2}} \circ f_{t_{2}, t_{1}}$. Then $F_{t_{2}, t_{1}} \circ f_{t_{1}, t_{0}}=F_{t_{2}, t_{0}}$ if $t_{0} \leq t_{1} \leq t_{2}$. Setting $t_{0}=0$ we get $F_{t_{2}, t_{1}} \circ f_{t_{1}}=F_{t_{2}}$. Thus, $F_{t_{2}, t_{1}}$ is a conformal map on $\mathbb{H}$ with $F_{t_{2}, t_{1}}\left(z_{t_{1}}\right)=0$ and $F_{t_{2}, t_{1}}^{\prime}\left(z_{t_{1}}\right)=1 / u_{t_{1}}$. By Koebe's distortion theorem, for any $t_{1} \in[0, T)$, $\left\{F_{t_{2}, t_{1}}: t_{2} \in\left[t_{1}, T\right)\right\}$ is uniformly bounded on each compact subset of $\mathbb{H}$. This implies that every sequence in this family contains a subsequence which converges locally uniformly, and the limit function is also conformal on $\mathbb{H}$, maps $z_{t_{1}}$ to 0 , and has derivative $1 / u_{t_{1}}$ at $z_{t_{1}}$.

From a diagonal argument, we can find a sequence $\left(t_{n}\right)$ in $[0, T)$ such that $t_{n} \rightarrow T$ and for any $q \in \mathbb{Q} \cap[0, T),\left(F_{t_{n}, q}\right)$ converges locally uniformly on $\mathbb{H}$. Let $F_{T, q}, q \in \mathbb{Q} \cap[0, T)$, denote the limit functions, which are conformal on $\mathbb{H}$. Since $F_{t_{n}, q_{2}} \circ f_{q_{2}, q_{1}}=F_{t_{n}, q_{1}}$ for each $n$, we have $F_{T, q_{2}} \circ f_{q_{2}, q_{1}}=F_{T, q_{1}}$. For $t \in[0, T)$, choose $q \in \mathbb{Q} \cap[t, T)$ and define the conformal map $F_{T, t}=F_{T, q} \circ f_{q, t}$ on $\mathbb{H}$. If $q_{1} \leq q_{2} \in \mathbb{Q} \cap[t, T)$, then $F_{T, q_{1}} \circ f_{q_{1}, t}=$ $F_{T, q_{2}} \circ f_{q_{2}, q_{1}} \circ f_{q_{1}, t}=F_{T, q_{2}} \circ f_{q_{2}, t}$. Thus, the definition of $F_{T, t}$ does not depend on the choice of $q$. If $0 \leq t_{1} \leq t_{2}<T$, by choosing $q \in \mathbb{Q} \cap[0, T)$ with $q \geq t_{1} \vee t_{2}$, we get $F_{T, t_{2}} \circ f_{t_{2}, t_{1}}=F_{T, q} \circ f_{q, t_{2}} \circ f_{t_{2}, t_{1}}=F_{T, q} \circ f_{q, t_{1}}=F_{T, t_{1}}$.

If (3.4) holds, then we start the construction of $\left(F_{T, t}\right)$ with such $z_{0}$. Since $F_{T, t}$ : $\left(\mathbb{H} ; z_{t}\right) \stackrel{\text { Conf }}{\rightarrow}\left(D_{t} ; 0\right)$ and $F_{T, t}^{\prime}\left(z_{t}\right)=1 / u_{t}$, Koebe's $1 / 4$ theorem implies that $\operatorname{dist}\left(0, \partial D_{t}\right) \geq$ $\frac{1}{4} \operatorname{Im} z_{t} /\left|u_{t}\right|=\frac{1}{4} \frac{\operatorname{Im} f_{t}\left(z_{0}\right)}{\left|f_{t}^{\prime}\left(z_{0}\right)\right|}$, which tends to $\infty$ as $t \rightarrow T$. So $D_{T}$ has to be $\mathbb{C}$.

Suppose $\widehat{F}_{T, t}, 0 \leq t<T$, satisfies the same property as $F_{T, t}, 0 \leq t<T$. Let $h_{t}=$ $\widehat{F}_{T, t} \circ F_{T, t}^{-1}, 0 \leq t<T$. Then each $h_{t}$ is a conformal map defined on $D_{t}$. If $0 \leq t_{1}<t_{2}<T$, then

$$
h_{t_{1}} \circ F_{T, t_{1}}=\widehat{F}_{T, t_{1}}(z)=\widehat{F}_{T, t_{2}} \circ f_{t_{2}, t_{1}}=h_{t_{2}} \circ F_{T, t_{2}} \circ f_{t_{2}, t_{1}}=h_{t_{2}} \circ F_{T, t_{1}}
$$

in $\mathbb{H}$, which implies that $h_{t_{1}}=\left.h_{t_{2}}\right|_{D_{t_{1}}}$. So we may define a conformal map $h_{T}$ on $D_{T}$ such that $h_{t}=\left.h_{T}\right|_{D_{t}}$ for $0 \leq t<T$. Such $h_{T}$ is what we need.

Suppose that 3.4 holds but $S_{T} \neq \mathbb{R}$. Since $S_{T}$ is an interval, $\overline{S_{T}} \neq \mathbb{R}$. Choose $\widehat{z}_{0} \in \mathbb{R} \backslash \overline{S_{T}}$, and start the construction with $\widehat{z}_{0}$ in place of $z_{0}$ at the beginning of this proof. Let $\widehat{F}_{T, t}, 0 \leq t<T$, denote the family of maps constructed in this way. Then each $\widehat{F}_{T, t}$ is an $\mathbb{R}$-symmetric conformal map, which implies that $\widehat{D}_{T} \subset \mathbb{H}$. However, now $D_{T}=\mathbb{C}$ and $h_{T}: D_{T} \stackrel{\text { Conf }}{\rightarrow} \widehat{D}_{T}$, which is impossible. Thus, $S_{T}=\mathbb{R}$ when 3.4 holds. 
Let $\left(F_{T, t}\right), D_{t}$, and $D_{T}$ be as in Lemma 3.6. Let $F_{T}=F_{T, 0}$. Suppose $\lambda$ generates backward chordal traces $\beta_{t_{0}}, 0 \leq t_{0}<T$, which satisfy

$$
\forall t_{0} \in[0, T), \quad \exists t_{1} \in\left(t_{0}, T\right), \quad \beta_{t_{1}}\left(\left[0, t_{0}\right]\right) \subset \mathbb{H} .
$$

We may define $\beta(t), 0 \leq t<T$, as follows. For every $t \in[0, T)$, pick $t_{0} \in(t, T)$ such that $\beta_{t_{0}}(t) \in \mathbb{H}$, which is possible by 3.5 , and define

$$
\beta(t)=F_{T, t_{0}} \beta_{t_{0}}(t) \in D_{t_{0}} \subset D_{T} .
$$

Since $F_{T, t_{1}}=F_{T, t_{2}} \circ f_{t_{2}, t_{1}}$ in $\mathbb{H}$, from $(3.3$ we see that the definition of $\beta$ does not depend on the choice of $t_{0}$. Let $t_{0} \in[0, T)$. From $(3.5)$, there is $t_{1}>t_{0}$ such that $\beta_{t_{1}}([0, t]) \in \mathbb{H}$. Since $\beta(t)=F_{T, t_{0}}\left(\beta_{t_{0}}(t)\right), 0 \leq t \leq t_{0}$, we see that $\beta$ is continuous on $\left[0, t_{0}\right]$. Thus, $\beta(t)$, $0 \leq t<T$, is a continuous curve in $D_{T}$.

Fix any $x \in S_{T}$. Then $x \in S_{t_{0}}$ for some $t_{0} \in(0, T)$. So $f_{t_{0}}(x)$ lies on the outer boundary of $L_{t_{0}}$, which implies that $f_{t_{0}}(x) \in \beta_{t_{0}}(t)$ for some $t \in\left[0, t_{0}\right]$. From $\left[3.5\right.$, there is $t_{1} \in\left(t_{0}, T\right)$ such that $\beta_{t_{1}}\left(\left[0, t_{0}\right]\right) \subset \mathbb{H}$. Then $f_{t_{1}}(x)=f_{t_{1}, t_{0}}\left(\beta_{t_{0}}(t)\right)=\beta_{t_{1}}(t) \in \mathbb{H}$. From the continuity of $f_{t_{1}}$ on $\mathbb{H} \cup \mathbb{R}$, there is a neighborhood $U$ of $x$ in $\mathbb{H} \cup \mathbb{R}$ such that $f_{t_{1}}(U) \subset \mathbb{H}$. This shows that $U \cap \mathbb{R} \subset S_{t_{1}} \subset S_{T}$. Since $F_{T}=F_{T, t_{1}} \circ f_{t_{1}}$ in $\mathbb{H}$, we find that $F_{T}$ has continuation on $U$. Since $x \in S_{T}$ is arbitrary, we conclude that $S_{T}$ is an open interval, and $F_{T}$ has continuation to $\mathbb{H} \cup S_{T}$.

Now we assume that $\lambda$ generates backward chordal traces, and both (3.4) and (3.5) hold. Then $D_{T}=\mathbb{C}, S_{T}=\mathbb{R}$, a continuous curve $\beta(t), 0 \leq t<T$, is well defined, and $F_{T}$ extends continuously to $\mathbb{H} \cup \mathbb{R}$. Moreover, $F_{T}$ is unique up to a Möbius transformation that fixes $\infty$. With some suitable normalization condition, the family $F_{T, t}$ and the curve $\beta$ will be determined by $\lambda$. We will use the following normalization:

$$
F_{T}(\lambda(0))=\lambda(0), \quad F_{T}(\lambda(0)+i)=\lambda(0)+i .
$$

If (3.7) holds, we call $\beta$ the normalized global backward chordal trace generated by $\lambda$. From 3.7 we see that $\beta(0)=\lambda(0)$, and $\beta$ does not pass through $\lambda(0)+i$.

For the radial case, Lemma 3.6 still holds with $\mathbb{H}$ replaced by $\mathbb{D}$, and (3.4) replaced by $T=\infty$. For the construction, we choose $z_{0}=0$ and let $F_{t_{2}, t_{1}}(z)=e^{t_{2}} \overline{f_{t_{2}, t_{1}}}(z)$. If $\lambda$ generates backward radial traces $\beta_{t}, 0 \leq t<T$, which satisfy

$$
\forall t_{1} \in[0, T), \quad \exists t_{2} \in\left(t_{1}, T\right), \quad \beta_{t_{2}}\left(t_{1}\right) \in \mathbb{D},
$$

then we may define a continuous curve $\beta(t), 0 \leq t<T$, in $D_{T}$ using $(3.6)$. If $T=\infty$, then $D_{T}=\mathbb{C}$, and such $\beta$ is determined by $\lambda$ up to a Möbius transformation that fixes $\infty$, which means that we may define a normalized global backward radial trace once a normalization condition is fixed.

\subsection{Forward and backward Loewner chains}

In this section, we review a condition on a family of hulls that corresponds to continuously driven (forward) Loewner hulls, and discuss the corresponding condition for backward Loewner chains.

Let $D \subset \widehat{\mathbb{C}}$ be a simply connected domain such that $\widehat{\mathbb{C}} \backslash D$ contains more than one point. A relatively closed subset $H$ of $D$ is called a (boundary) hull in $D$ if $D \backslash H$ is simply 
connected. For example, a hull in $\mathbb{H}$ is an $\mathbb{H}$-hull iff it is bounded; a hull in $\mathbb{D}$ is a $\mathbb{D}$-hull iff it does not contain 0 . Let $T \in(0, \infty]$. A family of hulls in $D: K_{t}, 0 \leq t<T$, is called a Loewner chain in $D$ if

1. $K_{0}=\emptyset$ and $K_{t_{1}} \varsubsetneqq K_{t_{2}}$ whenever $0 \leq t_{1}<t_{2}<T$;

2. for any fixed $a \in[0, T)$ and a compact set $F \subset D \backslash K_{a}$, the conjugate extremal distance (c.f. [1) between $K_{t+\varepsilon} \backslash K_{t}$ and $F$ in $D \backslash K_{t}$ tends to 0 as $\varepsilon \rightarrow 0$, uniformly in $t \in[0, a]$.

If $K_{t}, 0 \leq t<T$, is a Loewner chain in $D$, and $a \in[0, T)$, then we also call the restriction $K_{t}, 0 \leq t \leq a$, a Loewner chain in $D$.

There are two important properties for Loewner chains. If $K_{t}, 0 \leq t<T$, is a Loewner chain in $D$, and $u$ is a continuous increasing function defined on $[0, T)$ with $u(0)=0$, then $K_{u^{-1}(t)}, 0 \leq t<u(T)$, is also a Loewner chain in $D$, which is called a time-change of $\left(K_{t}\right)$ via $u$. If $W$ maps $D$ conformally onto $E$, then $W\left(K_{t}\right), 0 \leq t<T$, is a Loewner chain in $E$.

An $\mathbb{H}$-(resp. $\mathbb{D}$-)Loewner chain is a Loewner chain in $\mathbb{H}($ resp. $\mathbb{D})$ such that each hull is an $\mathbb{H}$-(resp. $\mathbb{D}$-) hull. An $\mathbb{H}$-(resp. $\mathbb{D}$-) Loewner chain $\left(K_{t}\right)$ is said to be normalized if $\operatorname{hcap}\left(K_{t}\right)=2 t\left(\operatorname{resp} \cdot \operatorname{dcap}\left(K_{t}\right)=t\right)$ for each $t$.

The conditions for the conformal invariance property of $\mathbb{H}$-(resp. $\mathbb{D}$-)Loewner chains can be slightly weakened as below.

Proposition 3.7. If $K_{t}, 0 \leq t<T$, is an $\mathbb{H}$-(resp. $\mathbb{D}$-)Loewner chain, and $W$ is an $\mathbb{R}$-(resp. $\mathbb{T}$-) symmetric conformal map, whose domain contains $\widehat{K}_{t}$ for each $t$ and whose image does not contain $\infty$ (resp. 0$)$, then $W\left(K_{t}\right), 0 \leq t<T$, is also an $\mathbb{H}$-(resp. $\mathbb{D}$-)Loewner chain.

The following proposition combines some results in [7] and [10].

Proposition 3.8. Let $T \in(0, \infty]$. The following are equivalent.

(i) $K_{t}, 0 \leq t<T$, are chordal (resp. radial) Loewner hulls driven by some $\lambda \in C([0, T)$ ).

(ii) $K_{t}, 0 \leq t<T$, is a normalized $\mathbb{H}$-(resp. $\mathbb{D}$-) Loewner chain.

If either of the above holds, with $\grave{\lambda}(t)=\lambda(t)$ (resp. $\stackrel{\lambda}{\lambda}(t)=e^{i \lambda(t)}$ in the radial case) we have

$$
\{\grave{\lambda}(t)\}=\bigcap_{\varepsilon>0} \overline{K_{t+\varepsilon} / K_{t}}, \quad 0 \leq t<T .
$$

In addition, if $K_{t}, 0 \leq t<T$, is any $\mathbb{H}$-(resp. $\mathbb{D}$-)Loewner chain, then the function $u(t):=$ $\operatorname{hcap}\left(K_{t}\right) / 2$ (resp. $\left.u(t):=\operatorname{dcap}\left(K_{t}\right)\right), 0 \leq t<T$, is continuous increasing with $u(0)=0$, which implies that $K_{u^{-1}(t)}, 0 \leq t<u(T)$, is a normalized $\mathbb{H}$-(resp. $\mathbb{D}$-) Loewner chain.

Definition 3.9. A family of $\mathbb{H}$-(resp. $\mathbb{D}$-)hulls: $L_{t}, 0 \leq t<T$, is called a backward $\mathbb{H}$-(resp. $\mathbb{D}$-) Loewner chain if they satisfy

1. $L_{0}=\emptyset$ and $L_{t_{1}} \prec L_{t_{2}}$ if $0 \leq t_{1} \leq t_{2}<T$;

2. $L_{t_{0}}: L_{t_{0}-t}, 0 \leq t \leq t_{0}$, is an $\mathbb{H}$-(resp. $\mathbb{D}$-)Loewner chain for any $t_{0} \in(0, T)$.

If $u$ is a continuous increasing function defined on $[0, T)$ with $u(0)=0$, then $L_{u^{-1}(t)}$, $0 \leq t<u(T)$, is also a backward $\mathbb{H}$-(resp. $\mathbb{D}$-)Loewner chain, and is called a time-change of $\left(L_{t}\right)$ via $u$. A backward $\mathbb{H}$-(resp. $\mathbb{D}$-)Loewner chain $\left(L_{t}\right)$ is said to be normalized if hcap $\left(L_{t}\right)=2 t\left(\operatorname{resp} . \operatorname{dcap}\left(L_{t}\right)=t\right)$ for any $t \in[0, T)$.

Using Lemma 3.3 and Proposition 3.8 , we obtain the following.

Proposition 3.10. Let $T \in(0, \infty]$. The following are equivalent. 
(i) $L_{t}, 0 \leq t<T$, are backward chordal (resp. radial) Loewner hulls driven by some $\lambda \in C([0, T))$.

(ii) $L_{t}, 0 \leq t<T$, is a normalized backward $\mathbb{H}$-(resp. $\mathbb{D}$-) Loewner chain.

If either of the above holds, with $\dot{\lambda}(t)=\lambda(t)$ (resp. $\dot{\lambda}(t)=e^{i \lambda(t)}$ in the radial case) we have

$$
\{\grave{\lambda}(t)\}=\bigcap_{\varepsilon>0} \overline{L_{t}: L_{t-\varepsilon}}, \quad 0<t<T,
$$

In addition, if $L_{t}, 0 \leq t<T$, is any backward $\mathbb{H}$-(resp. $\mathbb{D}$-) Loewner chain, then the function $u(t):=\operatorname{hcap}\left(K_{t}\right) / 2$ (resp. $\left.u(t):=\operatorname{dcap}\left(K_{t}\right)\right), 0 \leq t<T$, is continuous increasing with $u(0)=0$, which implies that $L_{u^{-1}(t)}, 0 \leq t<u(T)$, is a normalized backward $\mathbb{H}$-(resp. $\mathbb{D}$-) Loewner chain.

We say that $f_{t}$ and $L_{t}, 0 \leq t<T$, are backward chordal (resp. radial) Loewner maps and hulls, via a time-change $u$, driven by $\lambda$, if $u$ is a continuous increasing function defined on $[0, T)$ with $u(0)=0$, such that $f_{u^{-1}(t)}$ and $L_{u^{-1}(t)}, 0 \leq t<u(T)$, are backward chordal (resp. radial) Loewner maps and hulls driven by $\lambda \circ u^{-1}$. From the above proposition, if $\left(L_{t}\right)$ is any $\mathbb{H}$-(resp. $\mathbb{D}$-)Loewner chain, then $L_{t}, 0 \leq t<T$, are backward chordal (resp. radial) Loewner hulls, via a time-change $u(t):=\operatorname{hcap}\left(L_{t}\right) / 2\left(\operatorname{resp} . \operatorname{dcap}\left(L_{t}\right)\right)$, driven by $\lambda$, which satisfies 3.9 .

\subsection{Simple curves and weldings}

An $\mathbb{H}$-simple (resp. $\mathbb{D}$-simple) curve is a half-open simple curve in $\mathbb{H}$ (resp. $\mathbb{D} \backslash\{0\}$ ), whose open side approaches a single point on $\mathbb{R}$ (resp. $\mathbb{T})$. Every $\mathbb{H}($ resp. $\mathbb{D})$-simple curve $\beta$ is an $\mathbb{H}$ (resp. $\mathbb{D}$ )-hull, whose base $B_{\beta}$ is a single point, and whose support $S_{\beta}$ is an $\mathbb{R}$ (resp. $\mathbb{T}$-)interval. Here an $\mathbb{T}$-interval is an arc on $\mathbb{T}$. The function $f_{\beta}$ extends continuously from $\mathbb{H}($ resp. $\mathbb{D})$ to $\overline{\mathbb{H}}($ resp. $\overline{\mathbb{D}})$, which maps $S_{\beta}$ onto $\bar{\beta}$, sends the two ends of $S_{\beta}$ to $B_{\beta}$, and sends a unique point, say $z_{\beta} \in S_{\beta}$ to the tip of $\beta$. The point $z_{\beta}$ divides $S_{\beta}$ into two $\mathbb{R}$ (resp. $\mathbb{T}$-)intervals such that the restriction of $f_{\beta}$ to either interval is a homeomorphism onto $\bar{\beta}$. Thus, there is a unique involution (an auto homeomorphism whose inverse is itself) $\phi_{\beta}$ of $S_{\beta}$, which fixes only one point: $z_{\beta}$, swaps the two end points of $S_{\beta}$, and satisfies that $y=\phi_{\beta}(x)$ implies that $f_{\beta}(x)=f_{\beta}(y)$. We call $\phi_{\beta}$ the welding induced by $\beta$.

Suppose $K$ is an $\mathbb{H}$ - or $\mathbb{D}$-simple curve. Let $W$ be as in Theorems 2.12, 2.20, or 2.23. Then $W^{*}(K)$ is also an $\mathbb{H}$ - or $\mathbb{D}$-simple curve. The equality $W^{K} \circ f_{K}=f_{W^{*}(K)} \circ W$ holds after continuous extension from $\mathbb{H}$ or $\mathbb{D}$ to its closure. So the weldings induced by $K$ and $W^{*}(K)$ satisfy $\phi_{W^{*}(K)}=W \circ \phi_{K} \circ W^{-1}$.

Suppose the hulls $\left(L_{t}\right)$ generated by a backward chordal (resp. radial) Loewner process driven by $\lambda$ are all $\mathbb{H}($ resp. $\mathbb{D})$-simple curves. Then the process generates backward chordal (resp. radial) traces $\left(\beta_{t}\right)$ such that every $\beta_{t}$ is a simple curve, and $L_{t}=\beta_{t}([0, t)), 0 \leq t<T$. Let $\phi_{t}$ be the welding induced by $L_{t}$, which is an involution of $S_{t}:=S_{L_{t}}$. Recall that $\left(S_{t}\right)$ is an increasing family because $L_{t_{1}} \prec L_{t_{2}}$ for $t_{1}<t_{2}$. If $0 \leq t_{1}<t_{2}<T$, then from $f_{t_{2}, t_{1}} \circ f_{t_{1}}=f_{t_{2}}$ we see that $\left.\phi_{t_{2}}\right|_{S_{t_{1}}}=\phi_{t_{1}}$. Thus, there is a unique involution $\phi$ of $S_{T}:=\bigcup_{t} S_{t}$ such that $\left.\phi\right|_{S_{t}}=\phi_{t}$ for each $t \in[0, T)$. In other words, $y=\phi(x)$ implies that $f_{t}(x)=f_{t}(y)$ for some $t \geq 0$, where $f_{t}$ is the continuous extension of the Loewner map at time $t$ from $\mathbb{H}($ resp. $\mathbb{D})$ to $\overline{\mathbb{H}}$ (resp. $\overline{\mathbb{D}})$. We say that $\phi$ is the welding induced by this process. In the case that $S_{T}=\mathbb{R}$ (resp. $\mathbb{T} \backslash\left\{z_{0}\right\}$ for some $z_{0} \in \mathbb{T}$ ), we will extend $\phi$ to an involution of $\widehat{\mathbb{R}}:=\mathbb{R} \cup\{\infty\}\left(\right.$ resp. $\mathbb{T}$ ) such that $\infty$ (resp. $\left.z_{0}\right)$ is the other fixed point of $\phi$. 
Here is another way to view the welding $\phi$. For every $t \in(0, T), \phi$ swaps the two end points of $S_{t}$. Let $\grave{\lambda}(0)=\lambda(0)$ (resp. $\left.e^{i \lambda(0)}\right)$. Since $f_{t}(\stackrel{\circ}{\lambda}(0))=\beta_{t}(0)$ is the tip of $L_{t}$ for each $t$, we see that $\dot{\lambda}(0)$ is the only fixed point of $\phi$. On the other hand, it is easy to see that, $x$ and $y$ are end points of $S_{t}$ if and only if $\tau_{x}=\tau_{y}=t, 0<t<T$; and every point on

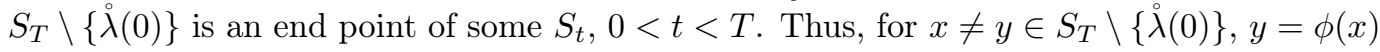
if and only if $\tau_{x}=\tau_{y}$, i.e., $x$ and $y$ are swallowed at the same time.

Let $\kappa \in(0,4]$. Since the backward chordal (resp. radial) $\mathrm{SLE}_{\kappa}$ traces are $\mathbb{H}$ (resp. $\mathbb{D}$ )simple curves, so the process induces a random welding, which we call a backward chordal (resp. radial) $\mathrm{SLE}_{\kappa}$ welding. In the chordal case, For any $x \in \mathbb{R} \backslash\{\lambda(0)\}=\mathbb{R} \backslash\{0\}$, the process $X_{t}^{x}:=\lambda(t)-f_{t}(x)$ is a rescaled Bessel process of dimension $1-\frac{4}{\kappa}<1$, which implies that a.s. $X_{t}^{x} \rightarrow 0$ at some finite time. Thus, $S_{\infty}=\mathbb{R}$. which implies that a chordal $\mathrm{SLE}_{\kappa}$ welding is an involution of $\widehat{\mathbb{R}}$ with two fixed points: $\lambda(0)=0$ and $\infty$. In the radial case, since $T=\infty$, Lemma 3.5 says that $S_{\infty}=\mathbb{T}$ or $\mathbb{T} \backslash\left\{z_{0}\right\}$ for some $z_{0} \in \mathbb{T}$. The first case can not happen since $\phi$ has only one fixed point on $S_{\infty}$. Thus, a radial $\mathrm{SLE}_{\kappa}$ welding is an involution of $\mathbb{T}$ with two fixed points, one of which is $e^{i \lambda(0)}=1$.

Suppose a backward chordal (resp. radial) Loewner process generates $\mathbb{H}($ resp. $\mathbb{D})$-simple backward traces $\beta_{t}, 0 \leq t<T$. Then (3.5) (resp. (3.8) is satisfied because $\beta_{t_{2}}\left(t_{1}\right)$ lies in $\mathbb{H}$ (resp. $\mathbb{D}$ ) if $t_{2}>t_{1}$. It is clear that the curve $\beta$ defined by $(3.6)$ is simple, and $D_{t}=D_{T} \backslash \beta([t, T))$ for $0 \leq t<T$. Let $\phi$ be the welding induced by the process. If $y=\phi(x)$, there is $t \in[0, T)$ such that $y, x \in S_{t}$ and $f_{t}(y)=f_{t}(x)$. From $F_{T, t} \circ f_{t}=F_{T}$, we get $F_{T}(y)=F_{T}(x)$. This means that $\phi$ can be realized by the conformal map $F_{T}$.

If a backward chordal (resp. radial) Loewner chain $\left(L_{t}\right)$ is composed of $\mathbb{H}($ resp. $\mathbb{D}$ )-simple curves, then $\left(L_{t}\right)$ induces a welding $\phi$, which is an involution of $\bigcup S_{L_{t}}$, and agrees with $\phi_{L_{t}}$ on $S_{L_{t}}$ for each $t$. To see this, one may first normalized the backward Loewner chain so that it is generated by a backward Loewner process.

\section{Conformal Transformations}

Proposition 4.1. Suppose $L_{t}, 0 \leq t<T$, is a backward $\mathbb{H}$-(resp. $\mathbb{D}$-) Loewner chain, $W$ is an $\mathbb{R}$-(resp. $\mathbb{T}$-)symmetric conformal map whose domain contains every $S_{L_{t}}$, and $\infty \notin W\left(S_{L_{t}}\right)$ for $0 \leq t<T$. Then $W^{*}\left(L_{t}\right), 0 \leq t<T$, is also a backward $\mathbb{H}$-(resp. $\mathbb{D}$-) Loewner chain.

Proof. From Theorem 2.12, $W^{L_{t}}$ and $W^{*}\left(L_{t}\right)$ are well defined. Since $L_{0}=\emptyset, W^{*}\left(L_{0}\right)=\emptyset$. Let $0 \leq t_{1} \leq t_{2}<T$. Since $L_{t_{1}} \prec L_{t_{2}}$, from Lemma 2.14. $W^{*}\left(L_{t_{1}}\right) \prec W^{*}\left(L_{t_{2}}\right)$. Fix $t_{0} \in(0, T)$. Since $L_{t_{0}}: L_{t_{0}-t}, 0 \leq t \leq t_{0}$, is an $\mathbb{H}$-(resp. $\mathbb{D}$-)Loewner chain, from Lemma 2.14 and Proposition 3.7 we see that

$$
W^{*}\left(L_{t_{0}}\right): W^{*}\left(L_{t_{0}-t}\right)=W^{L_{t_{0}}}\left(L_{t_{0}}: L_{t_{0}-t}\right), \quad 0 \leq t \leq t_{0},
$$

is also an $\mathbb{H}$-(resp. $\mathbb{D}$-)Loewner chain. This finishes the proof.

Using Lemma2.21 instead of Lemma 2.14, we can show that a similar proposition holds.

Proposition 4.2. Suppose $L_{t}, 0 \leq t<T$, is a backward $\mathbb{H}$-(resp. $\mathbb{D}$-)Loewner chain, $W$ is a Mobius transform that maps $\mathbb{H}$ onto $\mathbb{D}$ (resp. maps $\mathbb{D}$ onto $\mathbb{H})$ such that $\infty \notin W\left(S_{L_{t}}\right)$ for $0 \leq t<T$. Then $W^{*}\left(L_{t}\right), 0 \leq t<T$, is a backward $\mathbb{D}$-(resp. $\mathbb{H}$-)Loewner chain. 
Suppose $\left(L_{t}\right)$ is composed of $\mathbb{H}$ - or $\mathbb{D}$-simple curves. Then $\left(W^{*}\left(L_{t}\right)\right)$ is also composed of $\mathbb{H}$ or $\mathbb{D}$-simple curves. Let $\phi$ and $\phi_{W}$ be the weldings induced by these two chains, which are involutions of $\bigcup S_{L_{t}}$ and $\bigcup S_{W^{*}\left(L_{t}\right)}$, respectively. Since for each $t \in(0, T),\left.\phi\right|_{S_{L_{t}}}=\phi_{L_{t}}$, $\left.\phi_{W}\right|_{S_{W^{*}\left(L_{t}\right)}}=\phi_{W^{*}\left(L_{t}\right)}, S_{W^{*}\left(L_{t}\right)}=W\left(S_{L_{t}}\right)$, and $\phi_{W^{*}\left(L_{t}\right)}=W \circ \phi_{L_{t}} \circ W^{-1}$, we see that $\bigcup S_{W^{*}\left(L_{t}\right)}=W\left(\bigcup S_{L_{t}}\right)$ and

$$
\phi_{W}=W \circ \phi \circ W^{-1} .
$$

This means that the conformal transformation preserves the welding.

The following proposition is essentially Lemma 2.8 in [7.

Proposition 4.3. Let $W$ be an $\mathbb{R}$-symmetric conformal map, whose domain contains $z_{0} \in$ $\mathbb{R}$, such that $W\left(z_{0}\right) \neq \infty$. Then

$$
\lim _{H \rightarrow z_{0}} \frac{\operatorname{hcap}(W(H))}{\operatorname{hcap}(H)}=\left|W^{\prime}\left(z_{0}\right)\right|^{2},
$$

where $H \rightarrow z_{0}$ means that $\operatorname{diam}\left(H \cup\left\{z_{0}\right\}\right) \rightarrow 0$ with $H$ being a nonempty $\mathbb{H}$-hull.

Using the integral formulas for capacities of $\mathbb{H}$-hulls and $\mathbb{D}$-hulls, it is not hard to derive the following similar proposition.

Proposition 4.4. (i) Let $W$ be a conformal map on a $\mathbb{T}$-symmetric domain $\Omega$, which satisfies $I_{\mathbb{R}} \circ W=W \circ I_{\mathbb{T}}$ and $W(\Omega \cap \mathbb{D}) \subset \mathbb{H}$. Let $z_{0} \in \Omega \cap \mathbb{T}$ be such that $W\left(z_{0}\right) \neq \infty$. Then

$$
\lim _{H \rightarrow z_{0}} \frac{\operatorname{hcap}(W(H))}{\operatorname{dcap}(H)}=2\left|W^{\prime}\left(z_{0}\right)\right|^{2},
$$

where $H \rightarrow z_{0}$ means that $\operatorname{diam}\left(H \cup\left\{z_{0}\right\}\right) \rightarrow 0$ with $H$ being a nonempty $\mathbb{D}$-hull.

(ii) Proposition 4.3 holds with $\mathbb{R}$ replaced by $\mathbb{T}$, hcap replaced by dcap, and $H \rightarrow z_{0}$ understood as in (i).

\subsection{Transformations between backward $\mathbb{H}$-Loewner chains}

Suppose $L_{t}$ and $f_{t}, 0 \leq t<T$, are backward chordal Loewner hulls and maps driven by $\lambda \in C([0, T))$. From Proposition 3.10, $\left(L_{t}\right)$ is a backward $\mathbb{H}$-Loewner chain. Let $W$ be an $\mathbb{R}$-symmetric conformal map, whose domain $\Omega$ contains the support of every $L_{t}$. Write $W_{t}$ for $W^{L_{t}}$. The domain of $W_{t}$ is $\Omega^{L_{t}}$, which contains $\widehat{L}_{t}$. If $t>0, \lambda(t) \in \widehat{L}_{t}$, so $\lambda(t)$ is contained in the domain of $W_{t}$. This is also true for $t=0$ because $W_{0}=W$ and $\{\lambda(0)\}=S_{0} \subset S_{t}=S_{L_{t}} \subset \Omega$ for any $t \in(0, T)$. Let $L_{t}^{*}=W^{*}\left(L_{t}\right)=W_{t}\left(L_{t}\right), 0 \leq t<T$. From Proposition 4.1, $\left(L_{t}^{*}\right)$ is a backward $\mathbb{H}$-Loewner chain, and

$$
W_{t}\left(L_{t}: L_{t-\varepsilon}\right)=L_{t}^{*}: L_{t-\varepsilon}^{*}, \quad 0 \leq t-\varepsilon<t<T .
$$

From Proposition $3.10, L_{t}^{*}, 0 \leq t<T$, are backward chordal Loewner hulls via a time-change $u(t):=\operatorname{hcap}\left(L_{t}^{*}\right) / 2$, driven by some $\lambda^{*}$, which satisfies

$$
\left\{\lambda^{*}(t)\right\}=\bigcap_{\varepsilon>0} \overline{L_{t}^{*}: L_{t-\varepsilon}^{*}}, \quad 0<t<T .
$$

From 3.9 , 4.2, and continuity, we find that

$$
\lambda^{*}(t)=W_{t}(\lambda(t)), \quad 0 \leq t<T .
$$


Since $\left(L_{t}\right)$ and $\left(L_{u^{-1}(t)}^{*}\right)$ are normalized, we know that hcap $\left(L_{t}: L_{t-\varepsilon}\right)=2 \varepsilon$ and hcap $\left(L_{t}^{*}\right.$ : $\left.L_{t-\varepsilon}^{*}\right)=2 u(t)-2 u(t-\varepsilon)$. From 4.2 and Proposition 4.3 , we find that

$$
u^{\prime}(t)=W_{t}^{\prime}(\lambda(t))^{2}, \quad 0 \leq t<T .
$$

Let $f_{t}^{*}=f_{L_{t}^{*}}$. From the definition of $W_{t}=W^{L_{t}}$, we have the equality

$$
W_{t} \circ f_{t}=f_{t}^{*} \circ W
$$

which holds in $\Omega \backslash S_{L_{t}}$. Differentiating 4.5 w.r.t. $t$, and using 4.3 and (4.4), we get

$$
\begin{gathered}
\partial_{t} W_{t}\left(f_{t}(z)\right)+W_{t}^{\prime}\left(f_{t}(z)\right) \frac{-2}{f_{t}(z)-\lambda(t)}=\frac{-2 u^{\prime}(t)}{f_{t}^{*}(W(z))-\lambda^{*}(t)} \\
=\frac{-2 W_{t}^{\prime}(\lambda(t))^{2}}{W_{t}\left(f_{t}(z)\right)-W_{t}(\lambda(t))} .
\end{gathered}
$$

Thus, for any $w=f_{t}(z) \in f_{t}\left(\Omega \backslash S_{L_{t}}\right)=\Omega^{L_{t}} \backslash \widehat{L_{t}}$,

$$
\partial_{t} W_{t}(w)=\frac{-2 W_{t}^{\prime}(\lambda(t))^{2}}{W_{t}(w)-W_{t}(\lambda(t))}-W_{t}^{\prime}(w) \frac{-2}{w-\lambda(t)} .
$$

By analytic extension, the above equality holds for any $w \in \Omega^{L_{t}} \backslash\{\lambda(t)\}$. Letting $w \rightarrow \lambda(t)$, we find that

$$
\partial_{t} W_{t}(\lambda(t))=3 W_{t}^{\prime \prime}(\lambda(t)), \quad 0 \leq t<T .
$$

Differentiating (4.6) w.r.t. $w$ and letting $w \rightarrow \lambda(t)$, we get

$$
\frac{\partial_{t} W_{t}^{\prime}(\lambda(t))}{W_{t}^{\prime}(\lambda(t))}=-\frac{1}{2}\left(\frac{W_{t}^{\prime \prime}(\lambda(t))}{W_{t}^{\prime}(\lambda(t))}\right)^{2}+\frac{4}{3} \frac{W_{t}^{\prime \prime \prime}(\lambda(t))}{W_{t}^{\prime}(\lambda(t))} .
$$

\subsection{Transformations involving backward $\mathbb{D}$-Loewner chains}

Now suppose $L_{t}, 0 \leq t<T$, are backward radial Loewner hulls driven by $\lambda$. Let $f_{t}$ and $\widetilde{f}_{t}$ be the corresponding radial Loewner maps and covering maps. Suppose $W$ is a $\mathbb{T}$-symmetric conformal map, whose domain $\Omega$ contains the support of every $L_{t}$. Let $W_{t}=W^{L_{t}}, L_{t}^{*}=$ $W_{t}\left(L_{t}\right)=W^{*}\left(L_{t}\right)$, and $u(t)=\operatorname{dcap}\left(L_{t}^{*}\right), 0 \leq t<T$. Then $L_{t}^{*}, 0 \leq t<T$, are backward radial Loewner hulls via a time-change $u(t):=\operatorname{dcap}\left(L_{t}^{*}\right)$, driven by some $\lambda^{*}$, which satisfies

$$
\left\{e^{i \lambda^{*}(t)}\right\}=\bigcap_{\varepsilon>0} \overline{L_{t}^{*}: L_{t-\varepsilon}^{*}}, \quad 0<t<T .
$$

Let $f_{t}^{*}$ (resp. $\left.\widetilde{f}_{t}^{*}\right), 0 \leq t<T$, denote the backward radial (resp. covering radial) Loewner hulls via the time-change $u$ driven by $\lambda^{*}$. The argument in the last subsection still works with Proposition 4.4 in place of Proposition 4.3 . We can conclude that $e^{i \lambda(t)}$ lies in the domain of $W_{t}$ for $0 \leq t<T ; W_{t}\left(e^{i \lambda(t)}\right)=e^{i \lambda^{*}(t)} ; u^{\prime}(t)=\left|W_{t}^{\prime}\left(e^{i \lambda(t)}\right)\right|^{2} ;$ and 4.5 still holds. Suppose $\widetilde{W}$ is an $\mathbb{R}$-symmetric conformal map defined on $\widetilde{\Omega}=\left(e^{i}\right)^{-1}(\Omega)$, which satisfies $e^{i} \circ \widetilde{W}=W \circ e^{i}$. Define $\widetilde{W}_{t}$ to be the analytic extension of $\widetilde{f}_{t}^{*} \circ \widetilde{W} \circ \widetilde{f}_{t}^{-1}$ to $\widetilde{\Omega}_{t}:=\left(e^{i}\right)^{-1}\left(\Omega^{L_{t}}\right)$. Then we get

$$
\widetilde{W}_{t} \circ \widetilde{f}_{t}=\widetilde{f}_{t}^{*} \circ \widetilde{W}
$$


Comparing this with 4.5 we find $e^{i} \circ \widetilde{W}_{t}=W_{t} \circ e^{i}$. So $\lambda(t)$ lies in the domain of $\widetilde{W}_{t}$, and

$$
u^{\prime}(t)=\widetilde{W}_{t}^{\prime}(\lambda(t))^{2}, \quad 0 \leq t<T .
$$

Since $W_{t}\left(e^{i \lambda(t)}\right)=e^{i \lambda^{*}(t)}$, from the continuity, there is $n \in \mathbb{N}$ such that $\widetilde{W}_{t}(\lambda(t))=\lambda^{*}(t)+$ $2 n \pi$ for $0 \leq t<T$. Since $\lambda^{*}$ and $\lambda^{*}+2 n \pi$ generate the same backward radial Loewner objects via the time-change $u$, by replacing $\lambda^{*}$ with $\lambda^{*}+2 n \pi$, we may assume that

$$
\widetilde{W}_{t}(\lambda(t))=\lambda^{*}(t), \quad 0 \leq t<T .
$$

Differentiating 4.9 w.r.t. $t$ and letting $w=\widetilde{f}_{t}(z)$, we get

$$
\partial_{t} \widetilde{W}_{t}(w)=-\widetilde{W}_{t}^{\prime}(\lambda(t))^{2} \cot _{2}\left(\widetilde{W}_{t}(w)-\widetilde{W}_{t}(\lambda(t))\right)+\widetilde{W}_{t}^{\prime}(w) \cot _{2}(w-\lambda(t)),
$$

which holds for $w \in\left(e^{i}\right)^{-1}\left(\Omega^{L_{t}} \backslash\left\{e^{i \lambda(t)}\right\}\right)$. Letting $w \rightarrow \lambda(t)$, we get

$$
\partial_{t} \widetilde{W}_{t}(\lambda(t))=3 \widetilde{W}_{t}^{\prime \prime}(\lambda(t)), \quad 0 \leq t<T .
$$

Differentiating (4.12) w.r.t. $w$ and letting $w \rightarrow \lambda(t)$, we get

$$
\frac{\partial_{t} \widetilde{W}_{t}^{\prime}(\lambda(t))}{\widetilde{W}_{t}^{\prime}(\lambda(t))}=-\frac{1}{2}\left(\frac{W_{t}^{\prime \prime}(\lambda(t))}{W_{t}^{\prime}(\lambda(t))}\right)^{2}+\frac{4}{3} \frac{W_{t}^{\prime \prime \prime}(\lambda(t))}{W_{t}^{\prime}(\lambda(t))}+\frac{1}{6} \widetilde{W}_{t}^{\prime}(\lambda(t))^{2}-\frac{1}{6} .
$$

The number $\frac{1}{6}$ comes from the Laurent series of $\cot _{2}(z): \frac{2}{z}-\frac{z}{6}+O\left(z^{3}\right)$.

Let $\left(L_{t}\right),\left(f_{t}\right)$, and $\left(\widetilde{f}_{t}\right)$ be as above. Now suppose $W$ is a Möbius transformation that maps $\mathbb{D}$ onto $\mathbb{H}$ such that $W^{-1}(\infty) \notin S_{L_{t}}$ for every $t$. Let $W^{L_{t}}$ be as in Theorem 2.20 Let $W_{t}=W^{L_{t}}$ and $L_{t}^{*}=W_{t}\left(L_{t}\right)=W^{*}\left(L_{t}\right), 0 \leq t<T$. Then $L_{t}^{*}, 0 \leq t<T$, are backward chordal Loewner hulls via a time-change $u(t):=\operatorname{hcap}\left(L_{t}^{*}\right) / 2$, driven by some $\lambda^{*}$. Let $f_{t}^{*}=f_{L_{t}^{*}}$. Then 4.5$)$ still holds, and we have $u^{\prime}(t)=\left|W_{t}^{\prime}\left(e^{i \lambda(t)}\right)\right|^{2}$ and $W_{t}\left(e^{i \lambda(t)}\right)=e^{i \lambda^{*}(t)}$. Let $\widetilde{W}=W \circ e^{i}$ and $W_{t}=W_{t} \circ e^{i}$. We get 4.10, , 4.11), and $\widetilde{W}_{t} \circ \widetilde{f}_{t}=f_{t}^{*} \circ \widetilde{W}$. Differentiating this equality w.r.t. $t$ and letting $w=\widetilde{f}_{t}(z)$ tend to $\lambda(t)$, we find that 4.13 still holds.

\subsection{Conformal invariance of backward $\operatorname{SLE}(\kappa ; \rho)$ processes}

We now define backward chordal and radial $\operatorname{SLE}(\kappa ; \vec{\rho})$ processes, where $\vec{\rho}=\left(\rho_{1}, \ldots, \rho_{n}\right) \in$ $\mathbb{R}^{n}$. Let $x_{0}, q_{1}, \ldots, q_{n} \in \mathbb{R}$ such that $q_{k} \neq x_{0}$ for all $k$. Let $\lambda(t), 0 \leq t<T$, be the maximal solution of the equation

$$
d \lambda(t)=\sqrt{\kappa} d B(t)+\sum_{k=1}^{n} \frac{-\rho_{k}}{\lambda(t)-f_{t}^{\lambda}\left(q_{k}\right)} d t ; \quad \lambda(0)=x_{0} .
$$

Here $f_{t}^{\lambda}, 0 \leq t<T$, are the backward chordal Loewner maps driven by $\lambda$. Then we call the backward chordal Loewner process driven by $\lambda$ the chordal SLE $(\kappa ; \vec{\rho})$ process started from $x_{0}$ with force points $\left(q_{1}, \ldots, q_{n}\right)$, or simply started from $\left(x_{0} ; q_{1}, \ldots, q_{n}\right)$. We allow some $q_{k}$ to be $\infty$. In that case, $f_{t}^{\lambda}\left(q_{k}\right)$ is always $\infty$, and the term $\frac{-\rho_{k}}{\lambda(t)-f_{t}^{\lambda}\left(q_{k}\right)}$ vanishes.

Let $x_{0}, q_{1}, \ldots, q_{n} \in \mathbb{R}$ be such that $q_{k} \notin x_{0}+2 \pi \mathbb{Z}$ for all $k$. Let $\lambda(t), 0 \leq t<T$, be the maximal solution of the equation

$$
d \lambda(t)=\sqrt{\kappa} d B(t)+\sum_{k=1}^{n} \frac{-\rho_{k}}{2} \cot _{2}\left(\lambda(t)-\widetilde{f}_{t}^{\lambda}\left(q_{k}\right)\right) d t ; \quad \lambda(0)=x_{0} .
$$


Here $\widetilde{f}_{t}^{\lambda}, 0 \leq t<T$, are the covering backward radial Loewner maps driven by $\lambda$. Then the backward radial Loewner process driven by $\lambda$ is called the radial $\operatorname{SLE}(\kappa ; \vec{\rho})$ process started from $e^{i x_{0}}$ with marked points $\left(e^{i q_{1}}, \ldots, e^{i q_{n}}\right)$, or simply started from $\left(e^{i x_{0}} ; e^{i q_{1}}, \ldots, e^{i q_{n}}\right)$.

The existence of backward chordal (resp. radial) $\mathrm{SLE}_{\kappa}$ traces and Girsanov's Theorem imply the existence of a backward chordal (resp. radial) $\operatorname{SLE}(\kappa ; \vec{\rho})$ traces. The traces are $\mathbb{H}($ resp. $\mathbb{D})$-simple curves if $\kappa \in(0,4]$.

The following lemma is easy to check.

Lemma 4.5. Let $W$ be a Möbius transformation. Then the following hold.

(i) For any $z \in \mathbb{C} \cap W^{-1}(\mathbb{C})$ and $w \in \widehat{\mathbb{C}}$,

$$
\frac{2 W^{\prime}(z)}{W(z)-W(w)}-\frac{2}{z-w}=\frac{W^{\prime \prime}(z)}{W^{\prime}(z)} .
$$

(ii) Let $\widetilde{W}=W \circ e^{i}$. For any $z \in \mathbb{C} \cap \widetilde{W}^{-1}(\mathbb{C})$ and $w \in \mathbb{C}$,

$$
\frac{2 \widetilde{W}^{\prime}(z)}{\widetilde{W}(z)-\widetilde{W}(w)}-\cot _{2}(z-w)=\frac{\widetilde{W}^{\prime \prime}(z)}{\widetilde{W}^{\prime}(z)} .
$$

(iii) Suppose an analytic function $\widetilde{W}: \Omega \rightarrow \mathbb{C}$ satisfies $e^{i} \circ \widetilde{W}=W \circ e^{i}$ in $\Omega$. Then for any $z, w \in \Omega$,

$$
\widetilde{W}^{\prime}(z) \cot _{2}(\widetilde{W}(z)-\widetilde{W}(w))-\cot _{2}(z-w)=\frac{\widetilde{W^{\prime \prime}}(z)}{\widetilde{W}^{\prime}(z)} .
$$

Theorem 4.6. Let $L_{t}, 0 \leq t<T$, be the backward chordal $S L E(\kappa ; \vec{\rho})$ hulls started from $\left(x_{0} ; q_{1}, \ldots, q_{n}\right)$. Suppose $\sum \rho_{k}=-\kappa-6$. Let $W$ be a Möbius transformation from $\mathbb{H}$ onto $\mathbb{H}$ such that $\left\{\infty, W^{-1}(\infty)\right\} \subset\left\{q_{1}, \ldots, q_{n}\right\}$. Then, after a time-change, $W^{*}\left(L_{t}\right), 0 \leq t<T$, are the backward chordal $S L E(\kappa ; \vec{\rho})$ hulls started from $\left(W\left(x_{0}\right) ; W\left(q_{1}\right), \ldots, W\left(q_{n}\right)\right)$.

Proof. Since $W^{-1}(\infty)$ is a force point, it is not contained in the support of any $L_{t}$. So $\infty \notin W\left(S_{L_{t}}\right), 0 \leq t<T$. Let $\lambda$ be the driving function, and $f_{t}=f_{t}^{\lambda}, 0 \leq t<T$, be the corresponding maps. We may and now adopt the notation in Section 4.1. Let $\left(\mathcal{F}_{t}\right)$ be the complete filtration generated by $B(t)$ in $(4.15)$. Then $\left(\lambda_{t}\right)$ and $\left(L_{t}\right)$ are $\left(\mathcal{F}_{t}\right)$-adapted. From Corollary 2.19 (i), $\left(W^{*}\left(L_{t}\right)\right)$ is also $\left(\mathcal{F}_{t}\right)$-adapted. Since $W_{t}=W^{L_{t}}=f_{W^{*}\left(L_{t}\right)} \circ W \circ g_{L_{t}}$ on $\Omega^{L_{t}} \backslash \widehat{L_{t}},\left(W_{t}\right)$ is $\left(\mathcal{F}_{t}\right)$-adapted. So we may apply Itô's formula (c.f. [12]). From 4.3 and (4.7), we get

$$
d \lambda^{*}(t)=W_{t}^{\prime}(\lambda(t)) d \lambda(t)+\left(\frac{\kappa}{2}+3\right) W_{t}^{\prime \prime}(\lambda(t)) d t, \quad 0 \leq t<T .
$$

Applying 4.15 and Lemma 4.5 (i), and using the condition that $\sum \rho_{k}=-\kappa-6$, we find that

$$
\begin{aligned}
& d \lambda^{*}(t)=W_{t}^{\prime}(\lambda(t)) \sqrt{\kappa} d B(t)+\sum_{k=1}^{n} \frac{-\rho_{k} W_{t}^{\prime}(\lambda(t))^{2}}{W_{t}(\lambda(t))-W_{t} \circ f_{t}^{\lambda}\left(q_{k}\right)} d t \\
= & W_{t}^{\prime}(\lambda(t)) \sqrt{\kappa} d B(t)+\sum_{k=1}^{n} \frac{-\rho_{k} W_{t}^{\prime}(\lambda(t))^{2}}{\lambda^{*}(t)-f_{t}^{*} \circ W\left(q_{k}\right)} d t, \quad 0 \leq t<T .
\end{aligned}
$$


From (4.3) we get $\lambda^{*}(0)=W_{0}(\lambda(0))=W\left(x_{0}\right)$. Since $L_{t}^{*}=W^{*}\left(L_{t}\right)$ and $f_{t}^{*}$ are backward chordal Loewner hulls and maps via the time-change $u$ driven by $\lambda^{*}$, from (4.4) and the above equation, we conclude that, after a time-change, $W^{*}\left(L_{t}\right), 0 \leq t<T$, are the backward chordal SLE $(\kappa ; \vec{\rho})$ hulls started from $\left(W\left(x_{0}\right) ; W\left(q_{1}\right), \ldots, W\left(q_{n}\right)\right)$ and stopped at some time.

It remains to show that the above process is completed. If not, the process can be extended without swallowing the force points $W\left(q_{1}\right), \ldots, W\left(q_{n}\right)$. From the condition, $W(\infty)$ is among these force points. So $\left(W^{-1}\right)^{*}$ is well defined at the hulls of the extended process. From Propositions 4.1 and 3.10 , this implies that the backward chordal Loewner hulls $L_{t}, 0 \leq$ $t<T$, can be extended without swallowing any of $q_{1}, \ldots, q_{n}$, which is a contradiction.

The following theorem can be proved using the above proof with minor modifications: we now use the argument in Section 4.2 instead of that in Section 4.1, apply Lemma 4.5 (ii) and (iii) instead of (i), and use Proposition 4.2 in addition to Proposition 4.1 .

Theorem 4.7. Suppose $\sum \rho_{k}=-\kappa-6$. Let $\left(L_{t}\right)$ be the backward radial $S L E(\kappa ; \vec{\rho})$ hulls started from $\left(e^{i x_{0}} ; e^{i q_{1}}, \ldots, e^{i q_{n}}\right)$. Let $W$ map $\mathbb{D}$ conformally onto $\mathbb{H}$ (resp. $\left.\mathbb{D}\right)$ such that $\left\{W^{-1}(\infty)\right\} \cap \mathbb{T} \subset\left\{e^{i q_{1}}, \ldots, e^{i q_{n}}\right\}$. Then, after a time-change, $\left(W^{*}\left(L_{t}\right)\right)$ are the backward chordal (resp. radial) $S L E(\kappa ; \vec{\rho})$ hulls started from $\left(W\left(e^{i x_{0}}\right) ; W\left(e^{i q_{1}}\right), \ldots, W\left(e^{i q_{n}}\right)\right)$.

Corollary 4.8. Let $\left(L_{t}\right)$ be the backward radial SLE $(\kappa ;-\kappa-6)$ hulls started from $\left(e^{i x_{0}} ; e^{i q_{0}}\right)$. Let $W$ map $\mathbb{D}$ conformally onto $\mathbb{H}$ such that $W\left(e^{i x_{0}}\right)=0$ and $W\left(e^{i q_{0}}\right)=\infty$. Then, after a time-change, $\left(W^{*}\left(L_{t}\right)\right)$ are the backward chordal $S L E_{\kappa}$ hulls started from 0.

\section{Remarks.}

1. The above theorems resemble the work in [15] for forward $\operatorname{SLE}(\kappa ; \vec{\rho})$ processes. The condition in their paper is $\sum \rho_{k}=\kappa-6$. This is one reason why we may view backward $\mathrm{SLE}_{\kappa}$ as $\mathrm{SLE}_{-\kappa}$.

2. The definition of backward $\operatorname{SLE}(\kappa ; \vec{\rho})$ process differ from Sheffield's definition in [16] by a minus sign in (4.15) and (4.16) before the $\rho_{k}$ 's. If Sheffield's definition were used, the condition for conformal invariance would be $\sum \rho_{k}=\kappa+6$ instead of $\sum \rho_{k}=-\kappa-6$.

3. We may allow interior force points as in [15]. For the chordal (resp. radial) $\operatorname{SLE}(\kappa ; \vec{\rho})$ process, if $q_{k} \in \mathbb{H}\left(\right.$ resp. $\left.e^{i q_{k}} \in \mathbb{D}\right)$ is a force point, we use $\operatorname{Re} f_{t}^{\lambda}\left(q_{k}\right)\left(\operatorname{resp} . \operatorname{Re} \widetilde{f}_{t}^{\lambda}\left(q_{k}\right)\right)$ instead of $f_{t}^{\lambda}\left(q_{k}\right)$ (resp. $\left.\tilde{f}_{t}^{\lambda}\left(q_{k}\right)\right)$ in 4.15) (resp. 4.16). In the radial case, adding 0 to be a force point or change the force for 0 does not affect the process. Theorems 4.6 and Theorem 4.7 still hold if some or all force points lie inside $\mathbb{H}$ or $\mathbb{D}$. For the proofs, we apply Lemma 4.5 with real parts taken on the displayed formulas. One particular example is the following corollary.

Corollary 4.9. Let $L_{t}, 0 \leq t<\infty$, be a backward radial $S L E_{\kappa}$ process. Let $W$ be a Möbius transformation that maps $\mathbb{D}$ onto $\mathbb{H}$ such that $W(1) \neq \infty$. Let $T$ be the maximum number such that $W^{-1}(\infty) \notin S_{L_{t}}, 0 \leq t<T$. Then, after a time-change, $W^{*}\left(L_{t}\right), 0 \leq t<T$, are the backward chordal $S L E(\kappa ;-\kappa-6)$ hulls started from $(W(1) ; W(0))$.

4. Using the properties of Bessel process and applying Girsanov's theorem, one may define backward chordal or radial $\operatorname{SLE}(\kappa ; \vec{\rho})$ processes with exactly one degenerate force point, if the corresponding force $\rho_{1}$ satisfies $\rho_{1}<-2$ (which corresponds to a Bessel or Bessel-like process of dimension $d=1-\frac{4+2 \rho_{1}}{\kappa}>1$ ). Theorems 4.6 and 4.7 still hold when a degenerate force point exists. Unlike forward $\operatorname{SLE}(\kappa ; \vec{\rho})$ process, it is 
impossible to define a backward $\operatorname{SLE}(\kappa ; \vec{\rho})$ process with two different degenerate force points.

5. Consider the radial case with one force point. Suppose the force $\rho_{1} \leq-\frac{\kappa}{2}-2$. Let $X_{t}=\lambda(t)-\tilde{f}_{t}^{\lambda}\left(q_{1}\right)$. Then $X_{t}$ is a Bessel-like process with dimension $d=1-\frac{4+2 \rho_{1}}{\kappa} \geq 2$, which implies that $X_{t}$ never hits $2 \pi \mathbb{Z}$. So $T=\infty$ and $e^{i q_{1}} \notin S_{t}$ for any $t$. From Lemma 3.5. $S_{\infty}=\mathbb{T} \backslash\left\{e^{i q_{1}}\right\}$. If, in addition, $\kappa \in(0,4]$, then a backward radial $\operatorname{SLE}\left(\kappa ; \rho_{1}\right)$ process induces a random welding $\phi$, which is a involution of $\mathbb{T}$ with exactly two fixed point, $e^{i \lambda(0)}$ and $e^{i q_{1}}$, which are the initial point and the force point of the process.

\section{Commutation Relations}

Definition 5.1. Let $\kappa_{1}, \kappa_{2}>0, n \in \mathbb{N}$, and $\vec{\rho}_{1}, \vec{\rho}_{2} \in \mathbb{R}^{n}$. Let $z_{1}, z_{2}, w_{k}, 2 \leq k \leq n$, be distinct points on $\mathbb{R}$ (resp. $\mathbb{T})$. We say that a backward chordal (resp. radial) $S L E\left(\kappa_{1} ; \vec{\rho}_{1}\right)$ started from $\left(z_{1} ; z_{2}, w_{2}, \ldots, w_{n}\right)$ commutes with a backward chordal (resp. radial) $S L E\left(\kappa_{2} ; \vec{\rho}_{2}\right)$ started from $\left(z_{2} ; z_{1}, w_{2}, \ldots, w_{n}\right)$ if there exists a coupling of two processes $\left(L_{1}(t) ; 0 \leq t<T_{1}\right)$ and $\left(L_{2}(t) ; 0 \leq t<T_{2}\right)$ such that

(i) For $j=1,2,\left(L_{j}(t), 0 \leq t<T_{j}\right)$ is a complete backward chordal (resp. radial) $\operatorname{SLE}\left(\kappa_{j} ; \vec{\rho}_{j}\right)$ process started from $\left(z_{j} ; z_{3-j}, w_{2}, \ldots, w_{n}\right)$.

(ii) For $j \neq k \in\{1,2\}$, if $\bar{t}_{k}<T_{k}$ is a stopping time w.r.t. the complete filtration $\left(\mathcal{F}_{t}^{k}\right)$ generated by $\left(L_{k}(t)\right)$, then conditioned on $\mathcal{F}_{\bar{t}_{k}}^{k}$, after a time-change, $f_{k}\left(\bar{t}_{k}, \cdot\right)^{*}\left(L_{j}\left(t_{j}\right)\right)$, $0 \leq t_{j}<T_{j}\left(\bar{t}_{k}\right)$, has the distribution of a partial backward chordal (resp. radial) $S L E\left(\kappa_{j} ; \vec{\rho}_{j}\right)$ process started from

$$
\left(f_{k}\left(\bar{t}_{k},\left(z_{j}\right)\right) ; \stackrel{\lambda}{k}_{k}\left(\bar{t}_{k}\right), f_{k}\left(\bar{t}_{k}, w_{2}\right), \ldots, f_{k}\left(\bar{t}_{k}, w_{n}\right)\right),
$$

where $f_{k}\left(\bar{t}_{k}, \cdot\right):=f_{L_{k}\left(\bar{t}_{k}\right)}, T_{j}\left(\bar{t}_{k}\right):=\sup \left\{t_{j}<T_{j}: S_{L_{j}\left(t_{j}\right)} \cap S_{L_{k}\left(\bar{t}_{k}\right)}=\emptyset\right\}, \stackrel{\lambda}{k}_{k}\left(\bar{t}_{k}\right)=$ $\lambda_{k}\left(\bar{t}_{k}\right)$ in the chordal case (resp. $e^{i \lambda_{k}\left(\bar{t}_{k}\right)}$ in the radial case), and $\lambda_{k}$ is the driving function for $\left(L_{k}(t)\right)$.

Here a partial backward $S L E\left(\kappa ; \vec{\rho}_{j}\right)$ process is a complete $S L E\left(\kappa ; \vec{\rho}_{j}\right)$ process stopped at a positive stopping time. If the commutation holds for any distinct points $z_{1}, z_{2}, w_{k}, 2 \leq$ $k \leq n$ on $\mathbb{R}$ (resp. $\mathbb{T}$ ), then we simply say that backward chordal (resp. radial) $S L E\left(\kappa_{1} ; \vec{\rho}_{1}\right)$ commutes with backward chordal (resp. radial) $S L E\left(\kappa_{2} ; \vec{\rho}_{2}\right)$.

Theorem 5.2. For any $\kappa>0$, backward chordal (resp. radial) $S L E(\kappa ;-\kappa-6)$ commutes with backward chordal (resp. radial) SLE $(\kappa ;-\kappa-6)$.

We will prove this theorem in the next two subsections.

\section{$5.1 \quad$ Ensemble}

We first consider the radial case. Fix $\kappa>0$ and $z_{1} \neq z_{2} \in \mathbb{T}$. Write $z_{j}=e^{i \widetilde{z}_{j}}, j=1,2$. For $j=1,2$, let $L_{j}(t), 0 \leq t<T_{j}$, be a backward radial $\operatorname{SLE}(\kappa ;-\kappa-6)$ process started from $\left(z_{j} ; z_{3-j}\right)$; let $\lambda_{j}$ be the driving function, and let $f_{j}(t, \cdot)$ and $\widetilde{f}_{j}(t, \cdot), 0 \leq t<T_{j}$, be the corresponding maps and covering maps. At first, we suppose that the two processes are independent. Then for $j=1,2, \lambda_{j}$ satisfies $\lambda_{j}(0)=\widetilde{z}_{j}$ and the SDE:

$$
d \lambda_{j}(t)=\sqrt{\kappa} d B_{j}(t)-\frac{-\kappa-6}{2} \cot _{2}\left(\lambda_{j}(t)-\widetilde{f}_{j}\left(t, \widetilde{z}_{3-j}\right)\right) d t, \quad 0 \leq t<T_{j},
$$


where $B_{1}(t)$ and $B_{2}(t)$ are independent standard Brownian motions. For $j=1,2$, let $\left(\mathcal{F}_{t}^{j}\right)$ denote the complete filtration generated by $B_{j}(t)$.

Define $\mathcal{D}=\left\{\left(t_{1}, t_{2}\right) \in\left[0, T_{1}\right) \times\left[0, T_{2}\right): S_{L_{1}\left(t_{1}\right)} \cap S_{L_{2}\left(t_{2}\right)}=\emptyset\right\}$. Then for $\left(t_{1}, t_{2}\right) \in \mathcal{D}$, we have $\left(L_{1}\left(t_{1}\right), L_{2}\left(t_{2}\right)\right) \in \mathcal{P}_{*}$. So we may define

$$
\left(L_{1, t_{2}}\left(t_{1}\right), L_{2, t_{1}}\left(t_{2}\right)\right)=f^{*}\left(L_{1}\left(t_{1}\right), L_{2}\left(t_{2}\right)\right) .
$$

Let $f_{1, t_{2}}\left(t_{1}, \cdot\right)=f_{L_{1, t_{2}}\left(t_{1}\right)}$ and $f_{2, t_{1}}\left(t_{2}, \cdot\right)=f_{L_{2, t_{1}}\left(t_{2}\right)}$. From a radial version of Theorem 2.16. we see that

$$
f_{1, t_{2}}\left(t_{1}, \cdot\right) \circ f_{2}\left(t_{2}, \cdot\right)=f_{L_{1}\left(t_{1}\right) \vee L_{2}\left(t_{2}\right)}=f_{2, t_{1}}\left(t_{2}, \cdot\right) \circ f_{1}\left(t_{1}, \cdot\right) .
$$

Fix $j \neq k \in\{1,2\}$. From a radial version of Corollary 2.19 (ii), the random map $f_{j, t_{k}}\left(t_{j}, \cdot\right)$ is $\mathcal{F}_{t_{j}}^{j} \times \mathcal{F}_{t_{k}}^{k}$-measurable. Let $u_{j, t_{k}}\left(t_{j}\right)=\operatorname{dcap}\left(L_{j, t_{k}}\left(t_{j}\right)\right)$. From Propositions 3.10 and 4.1 . for any fixed $t_{k} \in\left[0, T_{k}\right), f_{j, t_{k}}\left(t_{j}, \cdot\right)$ are backward radial Loewner maps via the time-change $u_{j, t_{k}}$. Let $\widetilde{f}_{j, t_{k}}\left(t_{j}, \cdot\right)$ be the corresponding covering maps. So $e^{i} \circ \widetilde{f}_{j, t_{k}}\left(t_{j}, \cdot\right)=f_{j, t_{k}}\left(t_{j}, \cdot\right) \circ e^{i}$. From continuity, we see that $\widetilde{f}_{j, t_{k}}\left(t_{j}, \cdot\right)$ is also $\mathcal{F}_{t_{j}}^{j} \times \mathcal{F}_{t_{k}}^{k}$-measurable, and from 5.2 we have

$$
\widetilde{f}_{1, t_{2}}\left(t_{1}, \cdot\right) \circ \widetilde{f}_{2}\left(t_{2}, \cdot\right)=\widetilde{f}_{2, t_{1}}\left(t_{2}, \cdot\right) \circ \widetilde{f}_{1}\left(t_{1}, \cdot\right) .
$$

Define $\mathrm{m}$ on $\mathcal{D}$ by $\mathrm{m}\left(t_{1}, t_{2}\right)=\operatorname{dcap}\left(L_{1}\left(t_{1}\right) \vee L_{2}\left(t_{2}\right)\right)$. From 5.2 we get

$$
\mathrm{m}\left(t_{1}, t_{2}\right)=u_{1, t_{2}}\left(t_{1}\right)+t_{2}=u_{2, t_{1}}\left(t_{2}\right)+t_{1} .
$$

Apply the argument in the first paragraph of Section 4.2 with $\lambda=\lambda_{j}, L_{t_{j}}=L_{j}\left(t_{j}\right)$, $W=f_{k}\left(t_{k}, \cdot\right)$, and $\widetilde{W}=\widetilde{f}_{k}\left(t_{k}, \cdot\right)$, where $t_{k} \in\left[0, T_{k}\right)$ is fixed. Then we have correspondence: $L_{t_{j}}^{*}=L_{j, t_{k}}\left(t_{j}\right), u=u_{j, t_{k}}$, and $\widetilde{f}_{t_{j}}^{*}=\widetilde{f}_{j, t_{k}}\left(t_{j}, \cdot\right)$. Since $\widetilde{W}_{t_{j}}$ is an analytic extension of $\tilde{f}_{t_{j}}^{*} \circ \widetilde{W} \circ \widetilde{f}_{t_{j}}^{-1}$, from 5.3 , we find that $\widetilde{W}_{t_{j}}=\widetilde{f}_{k, t_{j}}\left(t_{k}, \cdot\right)$. Thus, $e^{i \lambda_{j}\left(t_{j}\right)}$ (resp. $\left.\lambda_{j}\left(t_{j}\right)\right)$ lies in the domain of $f_{k, t_{j}}\left(t_{k}, \cdot\right)$ (resp. $\left.\widetilde{f}_{k, t_{j}}\left(t_{k}, \cdot\right)\right)$ as long as $\left(t_{1}, t_{2}\right) \in \mathcal{D}$.

Write $\widetilde{F}_{k, t_{k}}\left(t_{j}, \cdot\right)=\widetilde{f}_{k, t_{j}}\left(t_{k}, \cdot\right)$. We will use $\partial_{t}$ to denote the partial derivative w.r.t. the first variable inside the parentheses, and use ' and the superscript $(h)$ to denote the partial derivatives w.r.t. the second variable inside the parentheses. For $h=0,1,2,3$, define $A_{j, h}$ on $\mathcal{D}$ by

$$
A_{j, h}\left(t_{1}, t_{2}\right)=\widetilde{f}_{k, t_{j}}^{(h)}\left(t_{k}, \lambda_{j}\left(t_{j}\right)\right)=\widetilde{F}_{k, t_{k}}^{(h)}\left(t_{j}, \lambda_{j}\left(t_{j}\right)\right) .
$$

Use the superscript $(S)$ to denote the (partial) Schwarzian derivative. Define $A_{j, S}$ on $\mathcal{D}$ by

$$
A_{j, S}\left(t_{1}, t_{2}\right)=\widetilde{f}_{k, t_{j}}^{(S)}\left(t_{k}, \lambda_{j}\left(t_{j}\right)\right)=\widetilde{F}_{k, t_{k}}^{(S)}\left(t_{j}, \lambda_{j}\left(t_{j}\right)\right)
$$

From Section 4.2 , we know that $L_{j, t_{k}}\left(t_{j}\right)$ are backward radial Loewner hulls via the timechange $u_{j, t_{k}}$ driven by $\lambda_{j, t_{k}}$, which can be chosen such that

$$
\lambda_{j, t_{k}}\left(t_{j}\right)=A_{j, 0}\left(t_{1}, t_{2}\right) .
$$

Moreover, from 4.10, 4.13, and 4.14, we have

$$
\begin{gathered}
u_{j, t_{k}}^{\prime}\left(t_{j}\right)=A_{j, 1}^{2}, \\
\partial_{t} \widetilde{F}_{k, t_{k}}\left(t_{j}, \lambda_{j}\left(t_{j}\right)\right)=3 A_{j, 2},
\end{gathered}
$$




$$
\frac{\partial_{t} \widetilde{F}_{k, t_{k}}^{\prime}\left(t_{j}, \lambda_{j}\left(t_{j}\right)\right)}{\widetilde{F}_{k, t_{k}}^{\prime}\left(t_{j}, \lambda_{j}\left(t_{j}\right)\right)}=-\frac{1}{2}\left(\frac{A_{j, 2}}{A_{j, 1}}\right)^{2}+\frac{4}{3} \frac{A_{j, 3}}{A_{j, 1}}+\frac{1}{6} A_{j, 1}^{2}-\frac{1}{6},
$$

where all $A_{j, h}$ are valued at $\left(t_{1}, t_{2}\right)$.

From now on, we fix an $\left(\mathcal{F}_{t}^{k}\right)$-stopping time $t_{k}$ with $t_{k}<T_{k}$. Then the process of

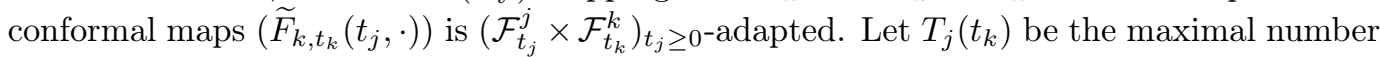
such that for any $t_{j}<T_{j}\left(t_{k}\right)$, we have $\left(t_{1}, t_{2}\right) \in \mathcal{D}$. Then $T_{j}\left(t_{k}\right)$ is an $\left(\mathcal{F}_{t_{j}}^{j} \times \mathcal{F}_{t_{k}}^{k}\right)_{t_{j} \geq 0}$-stopping time. Recall that $\left(\lambda_{j}(t)\right)$ is an $\left(\mathcal{F}_{t_{j}}^{j}\right)$-adapted local martingale with $\left\langle\lambda_{j}\right\rangle_{t}=\kappa t$. From now on, we will apply Itô's formula repeatedly. All SDEs below are $\left(\mathcal{F}_{t_{j}}^{j} \times \mathcal{F}_{t_{k}}^{k}\right)_{t_{j} \geq 0}$-adapted, and $t_{j}$ runs in the interval $\left[0, T_{j}\left(t_{k}\right)\right)$.

From (5.7), (5.5), and (5.9), we get

$$
d \lambda_{j, t_{k}}\left(t_{j}\right)=A_{j, 1} d \lambda_{j}\left(t_{j}\right)+\left(\frac{\kappa}{2}+3\right) A_{j, 2} d t, \quad 0 \leq t<T_{j}\left(t_{k}\right) .
$$

From $(5.5)$ and $(5.10)$ we get

$$
\frac{\partial_{t_{j}} A_{j, h}}{A_{j, h}}=\frac{A_{j, 2}}{A_{j, 1}} d \lambda_{j}+\left[-\frac{1}{2}\left(\frac{A_{j, 2}}{A_{j, 1}}\right)^{2}+\left(\frac{\kappa}{2}+\frac{4}{3}\right) \frac{A_{j, 3}}{A_{j, 1}}+\frac{1}{6} A_{j, 1}^{2}-\frac{1}{6}\right] d t_{j} .
$$

Let

$$
\alpha=\frac{6-(-\kappa)}{2(-\kappa)}, \quad \mathrm{c}=\frac{(8-3(-\kappa))(-\kappa-6)}{2(-\kappa)} .
$$

Note that if $-\kappa$ is replaced by $\kappa$, then c becomes the central charge for forward $\mathrm{SLE}_{\kappa}$. So we view the $\mathrm{c}$ here the central charge for backward $\mathrm{SLE}_{\kappa}$, which runs in the interval $[25, \infty)$. Since $A_{j, S}=\frac{A_{j, 3}}{A_{j, 1}}-\frac{3}{2}\left(\frac{A_{j, 2}}{A_{j, 1}}\right)^{2}$, from 5.12 we get

$$
\frac{\partial_{t_{j}} A_{j, 1}^{\alpha}}{A_{j, 1}^{\alpha}}=\alpha \frac{A_{j, 2}}{A_{j, 1}} d \lambda_{j}+\left[-\frac{\mathrm{c}}{6} A_{j, S}+\frac{\alpha}{6} A_{j, 1}^{2}-\frac{\alpha}{6}\right] d t_{j} .
$$

Now we study $\partial_{t_{j}} A_{k, h}$ and $\partial_{t_{j}} A_{k, S}$. From 5.5 we have $A_{k, h}\left(t_{1}, t_{2}\right)=\widetilde{F}_{j, t_{j}}^{(h)}\left(t_{k}, \lambda_{k}\left(t_{k}\right)\right)$. Recall that $\widetilde{F}_{j, t_{j}}\left(t_{k}, \cdot\right)=\widetilde{f}_{j, t_{k}}\left(t_{j}, \cdot\right)$, and $\widetilde{f}_{j, t_{k}}\left(t_{j}, \cdot\right)$ are backward covering radial Loewner maps via the time-change $u_{j, t_{k}}$ driven by $\lambda_{j, t_{k}}$. From (5.7) and (5.8), we get

$$
\partial_{t} \widetilde{f}_{j, t_{k}}\left(t_{j}, z\right)=-A_{j, 1}^{2} \cot _{2}\left(\widetilde{f}_{j, t_{k}}\left(t_{j}, z\right)-A_{j, 0}\right) .
$$

Differentiate the above formula w.r.t. $z$, we get

$$
\frac{\partial_{t}{\widetilde{f_{j, t_{k}}^{\prime}}}^{\prime}\left(t_{j}, z\right)}{{\widetilde{f_{j, t_{k}}^{\prime}}}_{(}\left(t_{j}, z\right)}=-A_{j, 1}^{2} \cot _{2}^{\prime}\left(\widetilde{f}_{j, t_{k}}\left(t_{j}, z\right)-A_{j, 0}\right) .
$$

Differentiating the above formula w.r.t. $z$, we get

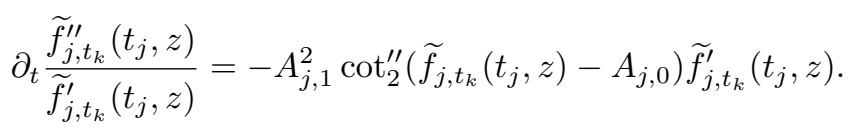

Since $f^{(S)}=\left(\frac{f^{\prime \prime}}{f^{\prime}}\right)^{\prime}-\frac{1}{2}\left(\frac{f^{\prime \prime}}{f^{\prime}}\right)^{2}$, from the above formula, we get

$$
\partial_{t} \widetilde{f}_{j, t_{k}}^{(S)}\left(t_{j}, z\right)=-A_{j, 1}^{2} \cot _{2}^{\prime \prime \prime}\left(\widetilde{f}_{j, t_{k}}\left(t_{j}, z\right)-A_{j, 0}\right) \widetilde{f}_{j, t_{k}}^{\prime}\left(t_{j}, z\right)^{2} .
$$


Letting $z=\lambda_{k}\left(t_{k}\right)$ in 5.14, (5.15), and 5.16), we get

$$
\begin{gathered}
\partial_{t_{j}} A_{k, 0}=-A_{j, 1}^{2} \cot _{2}\left(A_{k, 0}-A_{j, 0}\right) d t_{j} ; \\
\frac{\partial_{t_{j}} A_{k, 1}}{A_{k, 1}}=-A_{j, 1}^{2} \cot _{2}^{\prime}\left(A_{k, 0}-A_{j, 0}\right) d t_{j} \\
\partial_{t_{j}} A_{k, S}=-A_{j, 1}^{2} A_{k, 1}^{2} \cot _{2}^{\prime \prime \prime}\left(A_{k, 0}-A_{j, 0}\right) d t_{j} .
\end{gathered}
$$

Define $X_{j}$ on $\mathcal{D}$ such that $X_{j}=A_{j, 0}-A_{k, 0}$. Then $X_{1}+X_{2}=0$. Since $e^{i \lambda_{j}\left(t_{j}\right)}$ lies in the domain of $f_{k, t_{j}}\left(t_{k}, \cdot\right), e^{i A_{j, 0}}=f_{k, t_{j}}\left(t_{k}, e^{i \lambda_{j}\left(t_{j}\right)}\right)$ lies in the range of $f_{k, t_{j}}\left(t_{k}, \cdot\right)$, i.e., $\widehat{\mathbb{C}} \backslash L_{k, t_{j}}\left(t_{k}\right)$. On the other hand, since via a time-change, $L_{k, t_{j}}\left(t_{k}\right)$ are backward radial Loewner hulls driven by $\lambda_{k, t_{j}}\left(t_{k}\right)=A_{k, 0}$, from Lemma 3.4 we have $e^{i A_{k, 0}} \in L_{k, t_{j}}\left(t_{k}\right)$ when $t_{k}>0$. Thus, $e^{i A_{j, 0}} \neq e^{i A_{k, 0}}$ if $t_{k}>0$. Switching $j$ and $k$, the inequality also holds if $t_{j}>0$. If $t_{j}=t_{k}=0$, then $e^{i A_{j, 0}}=e^{i \widetilde{z}_{j}} \neq e^{i \widetilde{z}_{k}}=e^{i A_{k, 0}}$. Thus, $X_{j}, X_{k} \notin 2 \pi \mathbb{Z}$. So we may define

$$
Y=\left|\sin _{2}\left(X_{1}\right)\right|^{-2 \alpha}=\left|\sin _{2}\left(X_{2}\right)\right|^{-2 \alpha} .
$$

From 5.7), 5.11, and 5.17, we get

$$
\partial_{t_{j}} X_{j}=A_{j, 1} d \lambda_{j}+\left(\frac{\kappa}{2}+3\right) A_{j, 2} d t-A_{j, 1}^{2} \cot _{2}\left(X_{j}\right) d t .
$$

From Itô's formula, we get

$$
\begin{gathered}
\frac{\partial_{t_{j}} Y}{Y}=-\alpha \cot _{2}\left(X_{j}\right) A_{j, 1} d \lambda_{j}-\alpha\left(\frac{\kappa}{2}+3\right) A_{j, 2} \cot _{2}\left(X_{j}\right) d t_{j} \\
-\frac{\alpha}{2} A_{j, 1}^{2} \cot _{2}^{2}\left(X_{j}\right) d t_{j}+\frac{\alpha \kappa}{4} A_{j, 1}^{2} d t_{j} .
\end{gathered}
$$

Define $Q$ and $F$ on $\mathcal{D}$ such that $Q=\cot _{2}^{\prime \prime \prime}\left(X_{1}\right)=\cot _{2}^{\prime \prime \prime}\left(X_{2}\right)$ and

$$
F\left(t_{1}, t_{2}\right)=\exp \left(\int_{0}^{t_{2}} \int_{0}^{t_{1}} A_{1,1}\left(s_{1}, s_{2}\right)^{2} A_{2,1}\left(s_{1}, s_{2}\right)^{2} Q\left(s_{1}, s_{2}\right) d s_{1} d s_{2}\right) .
$$

Since $\widetilde{F}_{k, t_{k}}^{(S)}(0, \cdot)=$ id, from 5.6 we have $A_{j, S}=0$ when $t_{j}=0$. From 5.19 we get

$$
\frac{\partial_{t_{j}} F}{F}=-A_{j, S} d t_{j}
$$

Define a positive function $\widehat{M}$ on $\mathcal{D}$ by

$$
\widehat{M}=A_{1,1}^{\alpha} A_{2,1}^{\alpha} Y F^{-\frac{c}{6}} e^{\frac{c}{12} \mathrm{~m}} .
$$

From (5.4), 5.8), 5.13), 5.18, 5.20), and 5.22, we have

$$
\frac{\partial_{t_{j}} \widehat{M}}{\widehat{M}}=\alpha \frac{A_{j, 2}}{A_{j, 1}} d \lambda_{j}-\alpha \cot _{2}\left(X_{j}\right) A_{j, 1} d \lambda_{j}-\frac{\alpha}{6} d t_{j}
$$

When $t_{k}=0$, we have $A_{j, 1}=1, A_{j, 2}=0, \mathrm{~m}=t_{j}$, and $X_{j}=\lambda_{j}\left(t_{j}\right)-\tilde{f}_{j}\left(t_{j}, \widetilde{z}_{k}\right)$, so the RHS of (5.24) becomes

$$
\frac{1}{\kappa}\left(\frac{\kappa}{2}+3\right) \cot _{2}\left(\lambda_{j}\left(t_{j}\right)-\widetilde{f}_{j}\left(t_{j}, \widetilde{z}_{k}\right)\right) d \lambda_{j}-\frac{\alpha}{6} d t_{j} .
$$


Define another positive function $M$ on $\mathcal{D}$ by

$$
M\left(t_{1}, t_{2}\right)=\frac{\widehat{M}\left(t_{1}, t_{2}\right) \widehat{M}(0,0)}{\widehat{M}\left(t_{1}, 0\right) \widehat{M}\left(0, t_{2}\right)} .
$$

Then $M(\cdot, 0) \equiv M(0, \cdot) \equiv 1$. From (5.1), 5.24, and 5.25, we have

$$
\begin{gathered}
\frac{\partial_{t_{j}} M}{M}=\left[-\left(3+\frac{\kappa}{2}\right) \frac{A_{j, 2}}{A_{j, 1}}-\frac{-\kappa-6}{2} \cot _{2}\left(X_{j}\right) A_{j, 1}\right. \\
\left.+\frac{-\kappa-6}{2} \cot _{2}\left(\lambda_{j}\left(t_{j}\right)-\tilde{f}_{j}\left(t_{j}, \widetilde{z}_{k}\right)\right)\right] \frac{d B_{j}\left(t_{j}\right)}{\sqrt{\kappa}} .
\end{gathered}
$$

So when $t_{k} \in[0, p)$ is a fixed $\left(\mathcal{F}_{t}^{k}\right)$-stopping time, $M$ as a function in $t_{j}$ is an $\left(\mathcal{F}_{t_{j}}^{j} \times \mathcal{F}_{t_{k}}^{k}\right)_{t_{j} \geq 0^{-}}$ local martingale.

\subsection{Coupling measures}

Let JP denote the set of disjoint pairs of closed $\operatorname{arcs}\left(J_{1}, J_{2}\right)$ on $\mathbb{T}$ such that $z_{j}=e^{i \widetilde{z}_{j}}$ is contained in the interior of $J_{j}, j=1,2$. Let $T_{j}\left(J_{j}\right)$ denote the first time that $S_{L_{j}(t)}$ intersects $\overline{\mathbb{T} \backslash J_{j}}$. Then for every $\left(J_{1}, J_{2}\right) \in \mathrm{JP}$, if $t_{j} \leq T_{j}\left(J_{j}\right)$, then $S_{L_{j}\left(t_{j}\right)} \subset J_{j}$, which implies that $L_{j}\left(t_{j}\right) \in \mathcal{H}_{J_{j}}$. So $\left[0, T_{1}\left(J_{1}\right)\right] \times\left[0, T_{2}\left(J_{2}\right)\right] \subset \mathcal{D}$.

Proposition 5.3. (Boundedness) For any $\left(J_{1}, J_{2}\right) \in \mathrm{JP},|\ln (M)|$ is uniformly bounded on $\left[0, T_{1}\left(J_{1}\right)\right] \times\left[0, T_{2}\left(J_{2}\right)\right]$ by a constant depending only on $J_{1}$ and $J_{2}$.

Proof. Fix $\left(J_{1}, J_{2}\right) \in \mathrm{JP}$. In this proof, all constants depend only on $\left(J_{1}, J_{2}\right)$, and we say a function is uniformly bounded if its values on $\left[0, T_{1}\left(J_{1}\right)\right] \times\left[0, T_{2}\left(J_{2}\right)\right]$ are bounded in absolute value by a constant. From (5.23) and (5.26), it suffices to show that $\ln \left(A_{1,1}\right)$, $\ln \left(A_{2,1}\right), \ln (Y), \ln (F)$, and $m$ are all uniformly bounded.

Note that if $t_{j} \leq T_{j}\left(J_{j}\right)$, then $L_{j}\left(t_{j}\right) \in \mathcal{H}_{J_{j}}$. From a radial version of Theorem 2.16 (iii), we have

$$
\left\{L_{1}\left(t_{1}\right) \vee L_{2}\left(t_{2}\right): t_{j} \in\left[0, T_{j}\left(J_{j}\right)\right], j=1,2\right\} \subset \mathcal{H}_{J_{1} \cup J_{2}} .
$$

Since $J_{1} \cup J_{2} \varsubsetneqq \mathbb{T}$, from Lemma D.2 the righthand side is a compact set. So the lefthand side is relatively compact. Since $H \mapsto \operatorname{dcap}(H)$ is continuous, and $\mathrm{m}\left(t_{1}, t_{2}\right)=\operatorname{dcap}\left(L_{1}\left(t_{1}\right) \vee\right.$ $L_{2}\left(t_{2}\right)$ ), we see that $\mathrm{m}$ is uniformly bounded. For $j=1,2$, since $T_{j}\left(J_{j}\right) \leq \mathrm{m}, T_{j}\left(J_{j}\right)$ is also uniformly bounded.

Let $S_{1}$ and $S_{2}$ be the two components of $\mathbb{T} \backslash\left(J_{1} \cup J_{2}\right)$. For $s=1,2$, let $E_{s} \subset S_{s}$ be a compact arc. From Lemma D.3. $L_{n} \rightarrow L$ in $\mathcal{H}_{J_{1} \cup J_{2}}$ implies that $f_{L_{n}} \stackrel{\text { l.u. }}{\rightarrow} f_{L}$ in $\mathbb{C} \backslash\left(J_{1} \cup J_{2}\right)$, which then implies that $f_{L_{n}}^{\prime} \stackrel{\text { l.u. }}{\longrightarrow} f_{L}^{\prime}$ in $\mathbb{C} \backslash\left(J_{1} \cup J_{2}\right)$. From 5.28 , the compactness of $\mathcal{H}_{J_{1} \cup J_{2}}$, and that $E_{1} \cup E_{2}$ are compact subsets of $\mathbb{C} \backslash\left(J_{1} \cup J_{2}\right)$, we conclude that there is a constant $c_{1}>0$ such that $\left|f_{L_{1}\left(t_{1}\right) \vee L_{2}\left(t_{2}\right)}^{\prime}(z)\right| \geq c$ for any $t_{j} \leq T_{j}\left(J_{j}\right), j=1,2$, and $z \in E_{1} \cup E_{2}$. Thus, for $t_{j} \in\left[0, T_{j}\left(J_{j}\right)\right], j=1,2$, the length of $f_{L_{1}\left(t_{1}\right) \vee L_{2}\left(t_{2}\right)}\left(E_{s}\right), s=1,2$, is bounded below by a constant $c_{2}>0$. Suppose $t_{j} \in\left(0, T_{j}\left(t_{j}\right)\right], j=1,2$. From Lemma 3.4 , $e^{i A_{j, 0}} \in B_{L_{j, t_{3-j}}\left(t_{j}\right)}, j=1,2$. Note that $f_{L_{1}\left(t_{1}\right) \vee L_{2}\left(t_{2}\right)}\left(E_{1} \cup E_{2}\right)$ disconnects $B_{L_{1, t_{2}}\left(t_{1}\right)}$ from $B_{L_{2, t_{1}}\left(t_{2}\right)}$ on $\mathbb{T}$. Thus, there is a constant $c_{3}>0$ such that $\left|e^{i A_{1,0}\left(t_{1}, t_{2}\right)}-e^{i A_{2,0}\left(t_{1}, t_{2}\right)}\right| \geq c_{3}$ for $t_{j} \in\left(0, T_{j}\left(t_{j}\right)\right], j=1,2$. From continuity, this still holds if $t_{j} \in\left[0, T_{j}\left(J_{j}\right)\right], j=1,2$. Thus, $\ln (Y)=-2 \alpha \ln \left|\sin _{2}\left(X_{j}\right)\right|,\left|\cot _{2}^{\prime}\left(X_{j}\right)\right|$, and $\left|\cot _{2}^{\prime \prime \prime}\left(X_{j}\right)\right|, j=1,2$, are all uniformly bounded. 
We may find a Jordan curve $\sigma$, which is disjoint from $J_{1} \cup J_{2}$, such that its interior contains $J_{1}$ and its exterior contains $J_{2}$. From compactness, $\sup _{z \in \sigma} \ln \left|f_{j}^{\prime}\left(t_{j}, z\right)\right|$ and $\sup _{z \in \sigma} \ln \left|f_{L_{1}\left(t_{1}\right) \vee L_{2}\left(t_{2}\right)}^{\prime}(z)\right|$ are both uniformly bounded. From $(5.2)$ we see that the value $\sup _{w \in f_{j}\left(t_{j}, \sigma\right)} \ln \left|f_{3-j, t_{j}}^{\prime}\left(t_{k}, w\right)\right|$ is also uniformly bounded. Note that the interior of $f_{j}\left(t_{j}, \sigma\right)$ contains $\widehat{L_{j}\left(t_{j}\right)}$, which contains $e^{i \lambda_{j}\left(t_{j}\right)}$ if $t_{j}>0$. From maximal principle, there is $c_{4} \in$ $(0, \infty)$ such that $A_{j, 1}\left(t_{1}, t_{2}\right)=\left|f_{3-j, t_{j}}^{\prime}\left(t_{3-j}, e^{i \lambda_{j}\left(t_{j}\right)}\right)\right| \leq c_{4}$ if $t_{j} \in\left(0, T_{j}\left(J_{j}\right)\right]$ and $t_{3-j} \in$ $\left[0, T_{3-j}\left(J_{3-j}\right)\right]$. From continuity, $A_{j, 1}$ is uniformly bounded, $j=1,2$. From 5.18 and the uniformly boundedness of $\left|\cot _{2}^{\prime}\left(X_{j}\right)\right|$ we see that $\ln \left(A_{j, 1}\right)$ is uniformly bounded, $j=1,2$. From (5.21) and the uniformly boundedness of $\left|\cot _{2}^{\prime \prime \prime}\left(X_{j}\right)\right|$ we see that $\ln (F)$ is also uniformly bounded, which completes the proof.

Let $\mu_{j}$ denote the distribution of $\left(\lambda_{j}\right), j=1,2$. Let $\mu=\mu_{1} \times \mu_{2}$. Then $\mu$ is the joint distribution of $\left(\lambda_{1}\right)$ and $\left(\lambda_{2}\right)$, since $\lambda_{1}$ and $\lambda_{2}$ are independent. Fix $\left(J_{1}, J_{2}\right) \in$ JP. From the local martingale property of $M$ and Proposition 5.3 we have $\mathbf{E}_{\mu}\left[M\left(T_{1}\left(J_{1}\right), T_{2}\left(J_{2}\right)\right)\right]=M(0,0)=$ 1. Define $\nu_{J_{1}, J_{2}}$ by $d \nu_{J_{1}, J_{2}} / d \mu=M\left(T_{1}\left(J_{1}\right), T_{2}\left(J_{2}\right)\right)$. Then $\nu_{J_{1}, J_{2}}$ is a probability measure. Let $\nu_{1}$ and $\nu_{2}$ be the two marginal measures of $\nu_{J_{1}, J_{2}}$. Then $d \nu_{1} / d \mu_{1}=M\left(T_{1}\left(J_{1}\right), 0\right)=1$ and $d \nu_{2} / d \mu_{2}=M\left(0, T_{2}\left(J_{2}\right)\right)=1$, so $\nu_{j}=\mu_{j}, j=1,2$. Suppose temporarily that the joint distribution of $\left(\lambda_{1}\right)$ and $\left(\lambda_{2}\right)$ is $\nu_{J_{1}, J_{2}}$ instead of $\mu$. Then the distribution of each $\left(\lambda_{j}\right)$ is still $\mu_{j}$.

Fix an $\left(\mathcal{F}_{t}^{2}\right)$-stopping time $t_{2} \leq T_{2}\left(J_{2}\right)$. From (5.1), (5.27), and Girsanov theorem (c.f. [12]), under the probability measure $\nu_{J_{1}, J_{2}}$, there is an $\left(\mathcal{F}_{t_{1}}^{1} \times \mathcal{F}_{t_{2}}^{2}\right)_{t_{1} \geq 0}$-Brownian motion $\widetilde{B}_{1, t_{2}}\left(t_{1}\right)$ such that $\lambda_{1}\left(t_{1}\right), 0 \leq t_{1} \leq T_{1}\left(J_{1}\right)$, satisfies the $\left(\mathcal{F}_{t_{1}}^{1} \times \mathcal{F}_{t_{2}}^{2}\right)_{t_{1} \geq 0}$-adapted SDE:

$$
d \lambda_{1}\left(t_{1}\right)=\sqrt{\kappa} d \widetilde{B}_{1, t_{2}}\left(t_{1}\right)-\left(3+\frac{\kappa}{2}\right) \frac{A_{1,2}}{A_{1,1}} d t_{1}-\frac{-\kappa-6}{2} \cot _{2}\left(X_{1}\right) A_{1,1} d t_{1},
$$

which together with (5.5), (5.7), (5.9), and Itô's formula, implies that

$$
d \lambda_{1, t_{2}}\left(t_{1}\right)=A_{1,1} \sqrt{\kappa} d \widetilde{B}_{1, t_{2}}\left(t_{1}\right)-\frac{-\kappa-6}{2} \cot _{2}\left(X_{1}\right) A_{1,1}^{2} d t_{1} .
$$

From 5.5 and 5.7 we get $X_{1}=A_{1,1}-A_{2,1}=\lambda_{1, t_{2}}\left(t_{1}\right)-\tilde{f}_{1, t_{2}}\left(t_{1}, \lambda_{2}\left(t_{2}\right)\right)$. Note that $\lambda_{1, t_{2}}(0)=\widetilde{f}_{2,0}\left(t_{2}, \widetilde{z}_{1}\right)=\widetilde{f}_{2}\left(t_{2}, \widetilde{z}_{1}\right)$. Since $L_{1, t_{2}}\left(t_{1}\right)$ and $\widetilde{f}_{1, t_{2}}\left(t_{1}, \cdot\right)$ are backward radial Loewner hulls and covering maps via the time-change $u_{1, t_{2}}$, from (5.8) and the above equation, we find that, under the measure $\nu_{J_{1}, J_{2}}$, conditioned on $\mathcal{F}_{t_{2}}^{1}$ for any $\left(\mathcal{F}_{t}^{2}\right)$-stopping time $t_{2} \leq T_{2}\left(J_{2}\right)$, via the time-change $u_{1, t_{2}}, L_{1, t_{2}}\left(t_{1}\right)=f_{2}\left(t_{2}, \cdot\right)^{*}\left(L_{1}\left(t_{1}\right)\right), 0 \leq t_{1} \leq T_{1}\left(J_{1}\right)$, is a partial backward radial $\operatorname{SLE}\left(\kappa ; \frac{-\kappa-6}{2}\right)$ process started from $e^{i} \circ \widetilde{f}_{2}\left(t_{2}, \widetilde{z}_{1}\right)=f_{2}\left(t_{2}, z_{1}\right)$ with marked point $e^{i}\left(\lambda_{2}\left(t_{2}\right)\right)$. Similarly, the above statement holds true if the subscripts " 1 " and " 2 " are exchanged.

The joint distribution $\nu_{J_{1}, J_{2}}$ is a local coupling such that the desired properties in the statement of Theorem 5.2 holds true up to the stopping times $T_{1}\left(J_{1}\right)$ and $T_{2}\left(J_{2}\right)$. Then we can apply the maximum coupling technique developed in [18 to construct a global coupling using the local couplings within different pairs $\left(J_{1}, J_{2}\right)$. The reader is referred to Theorem 4.5 and Section 4.3 in [19] for the construction of a global coupling between two forward SLE processes. For the coupling of backward SLE processes, the method is essentially the same. A slight difference is that for the forward SLE processes, a pair of hulls were used to control the growth of $M(\cdot, \cdot)$, which stays uniformly bounded up to the time that the SLE hulls grow out of the given hulls; while for the backward SLE processes, we here used a pair 
of arcs to control the growth of $M(\cdot, \cdot)$. One fact that is worth mentioning is that here we may choose a dense countable set JP* $\subset$ JP such that, when $S_{L_{1}\left(t_{1}\right)} \cap S_{L_{2}\left(t_{2}\right)}=\emptyset$, there exists $\left(J_{1}, J_{2}\right) \in \mathrm{JP}^{*}$ with $S_{L_{j}\left(t_{j}\right)} \subset J_{j}, j=1,2$, from which follows that

$$
T_{j}\left(t_{k}\right)=\sup \left\{T_{j}\left(J_{j}\right):\left(J_{1}, J_{2}\right) \in \mathrm{JP}^{*}, t_{k} \leq T_{k}\left(J_{k}\right)\right\}, \quad j \neq k \in\{1,2\} .
$$

This finishes the proof of Theorem 5.2 in the radial case.

Now we briefly describe the proof for the chordal case. The proof in this case is simpler because there are no covering maps. Suppose the two backward chordal SLE $(\kappa ;-\kappa-6)$ processes start from $\left(z_{j} ; z_{k}\right)$, where $z_{1} \neq z_{2} \in \mathbb{R}$. Formula (5.1) holds with all tildes removed and the function $\cot _{2}$ replaced by $z \mapsto \frac{2}{z}$. The domain $\mathcal{D}$ and the $\mathbb{H}$-hulls $L_{1, t_{2}}\left(t_{1}\right)$ and $L_{2, t_{1}}\left(t_{2}\right)$ are defined in the same way. Then $(5.2)$ still holds. From Corollary 2.19 (ii), $f_{1, t_{2}}\left(t_{1}, \cdot\right)$ and $f_{2, t_{1}}\left(t_{2}, \cdot\right)$ are $\mathcal{F}_{t_{1}}^{1} \times \mathcal{F}_{t_{2}}^{2}$-measurable. Define $\mathrm{m}\left(t_{1}, t_{2}\right)=\operatorname{hcap}\left(L_{1}\left(t_{1}\right) \vee\right.$ $\left.L_{2}\left(t_{2}\right)\right) / 2$. Then (5.4) holds with $u_{j, t_{k}}\left(t_{j}\right):=\operatorname{hcap}\left(L_{j, t_{k}}\left(t_{j}\right)\right) / 2$.

Now we apply the argument in Section 4.1 with $W=f_{k}\left(t_{k}, \cdot\right)$. Then $W_{t}=f_{k, t_{j}}\left(t_{k}, \cdot\right)$. Let $F_{k, t_{k}}\left(t_{j}, \cdot\right)=f_{k, t_{j}}\left(t_{k}, \cdot\right)$, and define $A_{j, h}$ and $A_{j, S}$ using (5.5) and (5.6) with all tildes removed. Using (4.3), 4.4), 4.7), and (4.8), we see that (5.7) still holds here; (5.8) and (5.9) hold with all tildes removed; and 5.10 holds without the tildes and the terms $+\frac{1}{6} A_{j, 1}^{2}-\frac{1}{6}$. Then we get the SDEs 5.11) and (5.13) without the terms $+\frac{\alpha}{6} A_{j, 1}^{2}-\frac{\alpha}{6}$. Formulas (5.17), 5.18), and 5.19 hold with $\cot _{2}$ replaced by $z \mapsto \frac{2}{z}$. We still define $X_{j}=A_{j, 1}-A_{k, 1}$. Then $X_{j} \neq 0$ in $\mathcal{D}$. Define $Y$ on $\mathcal{D}$ by $Y=\left|X_{1}\right|^{-2 \alpha}=\left|X_{2}\right|^{-2 \alpha}$. Then $(5.20$ holds with $\cot _{2}$ replaced by $z \mapsto \frac{2}{z}$ and the term $+\frac{\alpha \kappa}{4} A_{j, 1}^{2} d t_{j}$ removed. Define $F$ using 5.21 with

$Q=-\frac{12}{X_{1}^{4}}=-\frac{12}{X_{2}^{4}}$. Then 5.22 still holds. Define $\widehat{M}$ using 5.23 without the factor $e^{\frac{c}{12} \mathrm{~m}}$. Then 5.24 holds with $\cot _{2}$ replaced by $z \mapsto \frac{2}{z}$ and the term $-\frac{\alpha}{6} d t_{j}$ removed. Define $M$ using (5.26). Then (5.27) holds with all tildes removed and $\cot _{2}$ replaced by $z \mapsto \frac{2}{z}$.

We define JP to be the set of disjoint pairs of closed real intervals $\left(J_{1}, J_{2}\right)$ such that $z_{j}$ is contained in the interior of $J_{j}$. Then Proposition 5.3 holds with a similar proof, where Lemma C.2 is applied here, and we can show that $\left|X_{1}\right|$ is uniformly bounded away from 0. The argument on the local couplings hold with all tildes and $e^{i}$ removed and $\cot _{2}$ replaced by $z \mapsto \frac{2}{z}$. Finally, we may apply the maximum coupling technique to construct a global coupling with the desired properties. Formula (5.29) still holds here and is used in the construction. This finishes the proof in the chordal case.

\subsection{Other results}

Besides Theorem 5.2, one may also prove the following two theorems, which are similar to the couplings for forward SLE that appear in [4] and [19].

Theorem 5.4. Let $\kappa_{1}, \kappa_{2}>0$ satisfy $\kappa_{1} \kappa_{2}=16$, and $c_{1}, \ldots, c_{n} \in \mathbb{R}$ satisfy $\sum_{k=1}^{n} c_{k}=\frac{3}{2}$. Let $\vec{\rho}_{j}=\left(\frac{\kappa_{j}}{2}, c_{1}\left(-\kappa_{j}-4\right), \ldots, c_{n}\left(-\kappa_{j}-4\right)\right), j=1,2$. Then backward chordal (resp. radial) $S L E\left(\kappa_{1} ; \vec{\rho}_{1}\right)$ commutes with backward chordal (resp. radial) $S L E\left(\kappa_{2} ; \vec{\rho}_{2}\right)$.

Theorem 5.5. Let $\kappa>0$ and $\vec{\rho} \in \mathbb{R}^{n}$, whose first coordinate is 2 . Then backward chordal (resp. radial) $S L E(\kappa ; \vec{\rho})$ commutes with backward chordal (resp. radial) $S L E(\kappa ; \vec{\rho})$. 


\section{Reversibility of Backward Chordal SLE}

Theorem 6.1. Let $\kappa \in(0,4]$ and $z_{1} \neq z_{2} \in \mathbb{T}$. Suppose a backward radial $S L E(\kappa ;-\kappa-6)$ process $\left(L_{1}(t)\right)$ started from $\left(z_{1} ; z_{2}\right)$ commutes with a backward radial $S L E(\kappa ;-\kappa-6)$ process $\left(L_{2}(t)\right)$ started from $\left(z_{2} ; z_{1}\right)$. Then a.s. they induce the same welding.

Proof. For $j=1,2$, let $S_{t}^{j}=S_{L_{j}(t)}$ and $f_{t}^{j}=f_{L_{j}(t)}$. Let $T_{j}(\cdot), j=1,2$, be as in Definition 5.1. Let $\phi_{j}$ be the welding induced by $\left(L_{j}(t)\right)$. Since $-\kappa-6 \leq-\kappa / 2-2$, from the last remark in Section 4.3 we see that, for $j=1,2$, a.s. $T_{j}=\infty, S_{\infty}^{j}=\mathbb{T} \backslash\left\{z_{3-j}\right\}$, and $\phi_{j}$ is an involution of $\mathbb{T}$ with exactly two fixed points: $z_{1}$ and $z_{2}$.

Fix $t_{2}>0$. Since $\left(L_{1}(t)\right)$ and $\left(L_{2}(t)\right)$ commute, the following is true. Conditioned on $\left(L_{2}(t)\right)_{t \leq t_{2}},\left(f_{t_{2}}^{2}\right)^{*}\left(L_{1}\left(t_{1}\right)\right), 0 \leq t_{1}<T_{1}\left(t_{2}\right)$, is a partial backward radial $\operatorname{SLE}(\kappa ;-\kappa-6)$ process, after a time-change, started from $\left(f_{t_{2}}^{2}\left(z_{1}\right) ; B_{L_{2}\left(t_{2}\right)}\right)$. Here we use $B_{L_{2}\left(t_{2}\right)}$ also to denote the unique point in the base of $L_{2}\left(t_{2}\right)$, which is equal to $e^{i \lambda_{2}\left(t_{2}\right)}$, where $\lambda_{2}$ is a driving function for $\left(L_{2}\left(t_{2}\right)\right)$. We have

$$
S:=\bigcup_{0 \leq t_{1}<T_{1}\left(t_{2}\right)} S_{\left(f_{t_{2}}^{2}\right)^{*}\left(L_{1}\left(t_{1}\right)\right)}=f_{t_{2}}^{2}\left(\bigcup_{0 \leq t_{1}<T_{1}\left(t_{2}\right)} S_{t_{1}}^{1}\right)=f_{t_{2}}^{2}\left(S_{T_{1}\left(t_{2}\right)^{-}}^{1}\right) .
$$

Recall that $f_{t_{2}}^{2}$ is a homeomorphism from $\mathbb{T} \backslash S_{t_{2}}^{2}$ onto $\mathbb{T} \backslash B_{L_{2}\left(t_{2}\right)}$. From the definition of $T_{1}\left(t_{2}\right)$, we see that $S_{T_{1}\left(t_{2}\right)}^{1}$ intersects $S_{t_{2}}^{2} \neq \emptyset$ at one or two end points of both arcs. If they intersect at only one point, then $S_{T_{1}\left(t_{2}\right)^{-}}^{1}$ is a proper subset of $\mathbb{T} \backslash S_{t_{2}}^{2}$, and these two arcs share an end point. From (6.1), this then implies that the arc $S$ is a proper subset of $\mathbb{T} \backslash B_{L_{2}\left(t_{2}\right)}$, and $B_{L_{2}\left(t_{2}\right)}$ is an end point of $S$. Recall that, after a time-change, $\left(f_{t_{2}}^{2}\right)^{*}\left(L_{1}\left(t_{1}\right)\right)$, $0 \leq t_{1}<T_{1}\left(t_{2}\right)$, is a partial backward radial $\operatorname{SLE}(\kappa ;-\kappa-6)$ process. Since $S \neq \mathbb{T} \backslash B_{L_{2}\left(t_{2}\right)}$, the process is not complete. Then we conclude that $S$ is contained in a closed arc on $\mathbb{T}$ that does not contain $B_{L_{2}\left(t_{2}\right)}$ because the force point is not swallowed by the process at any finite time, which contradicts that $B_{L_{2}\left(t_{2}\right)}$ is an end point of $S$. Thus, a.s. $S_{T_{1}\left(t_{2}\right)}^{1}$ and $S_{t_{2}}^{2}$ share two end points. Since $\phi_{j}$ swaps the two end points of any $S_{t}^{j}, j=1,2$, we see that a.s. $\phi_{2}=\phi_{1}$ on $\partial_{\mathbb{T}} S_{t_{2}}^{2}$. Let $t_{2}>0$ vary in the set of rational numbers, we see that a.s. $\phi_{2}=\phi_{1}$ on $\bigcup_{t \in \mathbb{Q}>0} \partial_{\mathbb{T}} S_{t_{2}}^{2}$, which is a dense subset of $\mathbb{T}$. The conclusion follows since $\phi_{1}$ and $\phi_{2}$ are continuous.

We now state the reversibility of backward chordal $\mathrm{SLE}_{\kappa}$ for $\kappa \in(0,4]$ in terms of its welding. Recall that a backward chordal $\mathrm{SLE}_{\kappa}$ welding is an involution of $\widehat{\mathbb{R}}$ with two fixed points: 0 and $\infty$.

Theorem 6.2. Let $\kappa \in(0,4]$, and $\phi$ be a backward chordal $S L E_{\kappa}$ welding. Let $h(z)=-1 / z$. Then $h \circ \phi \circ h$ has the same distribution as $\phi$.

Proof. Let $\left(L_{1}(t)\right)$ and $\left(L_{2}(t)\right)$ be commuting backward radial SLE $(\kappa ;-\kappa-6)$ procesees as in Theorem 5.2 which induce the weldings $\psi_{1}$ and $\psi_{2}$, respectively. The above theorem implies that a.s. $\psi_{1}=\psi_{2}$. For $j=1,2$, let $W_{j}$ be a Möbius transformation that maps $\mathbb{D}$ onto $\mathbb{H}$ such that $W_{j}\left(z_{j}\right)=0$ and $W_{j}\left(z_{3-j}\right)=\infty$, and $W_{2}=h \circ W_{1}$. From Corollary 4.8. $K_{j}(t):=W_{j}^{*}\left(L_{j}(t)\right), 0 \leq t<\infty$, is a backward chordal $\mathrm{SLE}_{\kappa}$, after a time-change, which then induces backward chordal $\mathrm{SLE}_{\kappa}$ welding $\phi_{j}, j=1,2$. Then $\phi_{1}$ and $\phi_{2}$ have the same law as $\phi$. From 4.1, we get $\phi_{j}=W_{j} \circ \psi_{j} \circ W_{j}^{-1}, j=1,2$, which implies that a.s. $\phi_{2}=h \circ \phi_{1} \circ h$. The conclusion follows since $\phi_{1}$ and $\phi_{2}$ has the same distribution as $\phi$. 
Lemma 6.3. Let $\kappa>0$. Let $f_{t}, 0 \leq t<\infty$, be backward chordal $S L E_{\kappa}$ maps. Then for every $z_{0} \in \mathbb{H}$, a.s. (3.4) holds.

Proof. Let $Z_{t}=f_{t}\left(z_{0}\right), X_{t}=\operatorname{Re} z_{t}$, and $Y_{t}=\operatorname{Im} z_{t}$. Then

$$
d X_{t}=-\sqrt{\kappa} d B(t)-\frac{2 X_{t}}{X_{t}^{2}+Y_{t}^{2}} d t, \quad d Y_{t}=\frac{2 Y_{t}}{X_{t}^{2}+Y_{t}^{2}} d t
$$

Let $R_{t}=\left|f_{t}^{\prime}\left(z_{0}\right)\right|$. Then $\frac{d R_{t}}{R_{t}}=\frac{2\left(X_{t}^{2}-Y_{t}^{2}\right)}{\left(X_{t}^{2}+Y_{t}^{2}\right)^{2}} d t$. Let $N_{t}=Y_{t} / R_{t}$ and $A_{t}=X_{t} / Y_{t}$. Then

$$
\frac{d N_{t}}{N_{t}}=\frac{4 Y_{t}^{2}}{\left(X_{t}^{2}+Y_{t}^{2}\right)^{2}} d t, \quad d A_{t}=-\frac{\sqrt{\kappa} d B(t)}{Y_{t}}-\frac{4 A_{t}}{X_{t}^{2}+Y_{t}^{2}} d t .
$$

Let $u(t)=\ln \left(Y_{t}\right)$. Then $u^{\prime}(t)=\frac{2}{X_{t}^{2}+Y_{t}^{2}}$. Let $T=\sup u([0, \infty))$ and define $\widehat{N}_{s}=N_{u^{-1}(s)}$ and $\widehat{A}_{s}=A_{u^{-1}(s)}$ for $0 \leq s<T$. Then

$$
\frac{d \widehat{N}_{s}}{\widehat{N}_{s}}=\frac{2}{\widehat{A}_{s}^{2}+1} d s, \quad d \widehat{A}_{s}=-\sqrt{1+\widehat{A}_{s}^{2}} \sqrt{\kappa / 2} d \widehat{B}(s)-2 \widehat{A}_{s} d s
$$

where $\widehat{B}(s)$ is another Brownian motion. We claim that $T=\infty$. Suppose $T<\infty$. Then $\lim _{t \rightarrow \infty} Y(t)=e^{T} \in \mathbb{R}$. From the SDE for $A_{s}$, we see that a.s. $\lim _{s \rightarrow T} A_{s} \in \mathbb{R}$, which implies that $\lim _{t \rightarrow \infty} A_{t} \in \mathbb{R}$ and $\lim _{t \rightarrow \infty} X_{t} \in \mathbb{R}$ as $X_{t}=Y_{t} A_{t}$. Then we have a.s. $s^{\prime}(t)=\frac{2}{X_{t}^{2}+Y_{t}^{2}}$ tends to a finite positive number as $t \rightarrow \infty$, which contradicts that $T=\sup \{s(t), 0 \leq$ $t<\infty\}<\infty$. So the claim is proved. Using Itô's formula, we see that $\widehat{A}_{s}, 0 \leq s<\infty$, is recurrent. Since $\left(\ln \left(\widehat{N}_{s}\right)\right)^{\prime}=\frac{2}{\widehat{A}_{s}^{2}+1}$, we see that a.s. $\widehat{N}_{s} \rightarrow \infty$ as $s \rightarrow \infty$. So a.s. $N_{t}=\frac{\operatorname{Im} f_{t}\left(z_{0}\right)}{\left|f_{t}^{\prime}\left(z_{0}\right)\right|} \rightarrow \infty$ as $t \rightarrow \infty$, i.e., 3.4 holds.

If $\kappa \in(0,4]$, then since the backward chordal traces are simple, 3.5$)$ holds. From the above lemma and Section 3.3 , we see that, for $\kappa \in(0,4]$, the backward chordal $\mathrm{SLE}_{\kappa}$ a.s. generates a normalized global backward chordal trace $\beta$, which we call a normalized global backward chordal $\mathrm{SLE}_{\kappa}$ trace. Recall that $\beta(t), 0 \leq t<\infty$, is simple with $\beta(0)=0$, and $i \notin \beta$; and there is $F_{\infty}: \mathbb{H} \stackrel{\text { Conf }}{\rightarrow} \mathbb{C} \backslash \beta$, whose continuation maps $\mathbb{R}$ onto $\beta$ such that 3.7 . holds, and for any $x \in \mathbb{R}, F_{\infty}(x)=F_{\infty}(\phi(x)) \in \beta$. Now we state the reversibility of the backward chordal $\mathrm{SLE}_{\kappa}$ for $\kappa \in(0,4)$ in terms of $\beta$.

Theorem 6.4. Let $\kappa \in(0,4)$, and $\beta$ be a normalized global backward chordal SLE $E_{\kappa}$ trace. Let $h(z)=-1 / z$. Then $h(\beta \backslash\{0\})$ has the same distribution as $\beta \backslash\{0\}$ as random sets.

Proof. For $j=1,2$, let $\phi_{j}$ be a backward chordal $\mathrm{SLE}_{\kappa}$ welding and $\beta_{j}$ be the corresponding normalized global trace. Then $\beta_{j}$ is a simple curve with one end point 0 , and there exists $F_{j}: \mathbb{H} \stackrel{\text { Conf }}{\rightarrow} \mathbb{C} \backslash \beta_{j}$ such that $F_{j}(i)=i, F_{j}(0)=0$, and $F_{j}(x)=F_{j}\left(\phi_{j}(x)\right)$ for $x \in \mathbb{R}$. From Theorem 6.2 we may assume that $\phi_{2}=h \circ \phi_{1} \circ h^{-1}$. Now it suffices to show that $h\left(\beta_{2} \backslash\{0\}\right)=\beta_{1} \backslash\{0\}$.

Define $G=h \circ F_{2} \circ h \circ F_{1}^{-1}$. Then $G$ is a conformal map defined on $\mathbb{C} \backslash \beta_{1}$. It has continuation to $\beta_{1} \backslash\{0\}$. In fact, if $z \in \mathbb{C} \backslash \beta_{1}$ and $z \rightarrow z_{0} \in \beta_{1} \backslash\{0\}$, then $F_{1}^{-1}(z) \rightarrow\left\{x, \phi_{1}(x)\right\}$ for some $x \in \mathbb{R} \backslash\{0\}$, which then implies that $h \circ F_{1}^{-1}(z) \rightarrow\left\{h(x), h \circ \phi_{1}(x)\right\}$; since 
$\phi_{2} \circ h=h \circ \phi_{1}$, we find that $F_{2} \circ h \circ F_{1}^{-1}(z)$ tends to some point on $\beta_{2} \backslash\{0\}$, so $G(z)$ tends to some point on $h\left(\beta_{2} \backslash\{0\}\right)$. It was proved in [13] that a forward $\mathrm{SLE}_{\kappa}$ trace is the boundary of a Hölder domain. Then the same is true for backward chordal SLE $\mathrm{K}_{\kappa}$ traces and the normalized global trace. From the results in [5], we see that $\beta_{1} \backslash\{0\}$ is conformally removable, which means that $G$ extends to a conformal map from $\left(\mathbb{C} \backslash \beta_{1}\right) \cup\left(\beta_{1} \backslash\{0\}\right)=\mathbb{C} \backslash\{0\}$ onto $\mathbb{C} \backslash\{0\}$, and maps $\beta_{1} \backslash\{0\}$ to $h\left(\beta_{2} \backslash\{0\}\right)$. Since $G(i)=i$, either $G=\operatorname{id}$ or $G=h$. Suppose $G=h$. Then $F_{1}=F_{2} \circ h$. Since $F_{1}(0)=F_{2}(0)=0$, for $j=1,2, F_{j}$ maps a neighborhood of 0 in $\mathbb{H}$ onto a neighborhood of 0 in $\mathbb{C}$ without a simple curve. Since $F_{1}=F_{2} \circ h, F_{1}$ also maps a neighborhood of $\infty$ in $\mathbb{H}$ onto a neighborhood of 0 without a simple curve, which contradicts the univalent property of $F_{1}$. Thus, $G=$ id, and we get $h\left(\beta_{2} \backslash\{0\}\right)=G\left(\beta_{1} \backslash\{0\}\right)=\beta_{1} \backslash\{0\}$, as desired.

Now we propose a couple of questions. First, let's consider backward chordal $\mathrm{SLE}_{\kappa}$ for $\kappa>4$. Since the process does not generate simple backward chordal traces, the random welding $\phi$ can not be defined. However, the lemma below and the discussion in Section 3.3 show that we can still define a global backward chordal $\mathrm{SLE}_{\kappa}$ trace.

Lemma 6.5. Let $\kappa \in(0, \infty)$. Suppose $\beta_{t}, 0 \leq t<\infty$, are backward chordal traces driven by $\lambda(t)=\sqrt{\kappa} B(t)$. Then a.s. 3.5) holds.

Proof. If $\kappa \in(0,4]$, a.s. the traces are simple, so 3.5 holds. Now suppose $\kappa>4$. Let $f_{t}$ and $L_{t}$ be the corresponding maps and hulls. It suffices to show that, for any $t_{0}>0$, a.s. there exists $t_{1}>t_{0}$ such that $\beta_{t_{1}}\left(\left[0, t_{0}\right]\right) \subset \mathbb{H}$.

Let $g_{t}$ and $K_{t}, 0 \leq t<\infty$, be the forward chordal Loewner maps and hulls driven by $\sqrt{\kappa} B(t)$. From Theorem 6.1 in [20, for any deterministic time $t_{1} \in(0, \infty)$, the continuation of $g_{t_{1}}^{-1}$ a.s. maps the interior of $S_{K_{t_{1}}}$ into $\mathbb{H}$. From Lemma 3.1 and the property of Brownian motion, we see that, for any $t_{1} \in(0, \infty), f_{t_{1}}$ has the same distribution as $\lambda\left(t_{1}\right)+g_{t_{1}}^{-1}\left(\cdot-\lambda\left(t_{1}\right)\right)$, which implies that the continuation of $f_{t_{1}}$ a.s. maps the interior of $S_{L_{t_{1}}}$ into $\mathbb{H}$.

Since a.s. $\bigcup_{n=1}^{\infty} S_{n}=S_{\infty}=\mathbb{R} \supset \lambda\left(\left[0, t_{0}\right]\right)$, and $\left(S_{t}\right)$ is an increasing family of intervals, we see that a.s. there is $N \in \mathbb{N}$ such that the interior of $S_{N}$ contains $\lambda\left(\left[0, t_{0}\right]\right)$. Let $t_{1}=N$. Then $f_{t_{1}}$ maps $\lambda\left(\left[0, t_{0}\right]\right)$ into $\mathbb{H}$, which implies that $\beta_{t_{1}}(t)=f_{t_{1}}(\lambda(t)) \in \mathbb{H}$ for $0 \leq t \leq t_{0}$.

Question 6.6. Do we have the reversibility of the global backward chordal SLE $E_{\kappa}$ trace for $\kappa>4$ ?

Second, let's consider backward radial $\mathrm{SLE}_{\kappa}$ processes. One can show that 3.8 a.s. holds. Since $T=\infty$, we may define a global backward radial $\mathrm{SLE}_{\kappa}$ trace.

Question 6.7. Does a global backward radial $S L E_{\kappa}$ trace satisfy some reversibility property of any kind?

Recall that the forward radial $\mathrm{SLE}_{\kappa}$ trace does not satisfy the reversibility property in the usual sense. However, it's proved in [22] that, for $\kappa \in(0,4]$, the whole-plane $\mathrm{SLE}_{\kappa}$, as a close relative of radial $\mathrm{SLE}_{\kappa}$, satisfies reversibility.

Finally, it is worth mentioning the following simple fact. Recall that, if $\kappa \in(0,4]$, a backward radial $\mathrm{SLE}_{\kappa}$ welding is an involution of $\mathbb{T}$ with two fixed points, one of which is 1. The following theorem gives the distribution of the other fixed point $\zeta$, and says that a backward radial $\mathrm{SLE}_{\kappa}$ process conditioned on $\zeta$ is a backward radial $\operatorname{SLE}(\kappa ;-4)$ process with force point $\zeta$. It is similar to Theorem 3.1 in [20], and we omit its proof. 
Theorem 6.8. Let $\kappa \in(0,4]$. Let $\mu$ denote the distribution of a backward radial SLE $E_{\kappa}$ process. For $\theta \in(0,2 \pi)$, let $\nu_{\theta}$ denote the distribution of a backward radial $S L E(\kappa ;-4)$ process started from $\left(1 ; e^{i \theta}\right)$. Let $f(\theta)=C \sin _{2}(\theta)^{4 / \kappa}$, where $C>0$ is such that $\int_{0}^{2 \pi} f(\theta) d \theta=$ 1. Then

$$
\mu=\int_{0}^{2 \pi} \nu_{\theta} f(\theta) d \theta
$$

\section{Appendices}

\section{A Carathéodory Topology}

Definition A.1. Let $\left(D_{n}\right)_{n=1}^{\infty}$ and $D$ be domains in $\mathbb{C}$. We say that $\left(D_{n}\right)$ converges to $D$, and write $D_{n} \stackrel{\text { Cara }}{\longrightarrow} D$, if for every $z \in D$, $\operatorname{dist}\left(z, \mathbb{C} \backslash D_{n}\right) \rightarrow \operatorname{dist}(z, \mathbb{C} \backslash D)$. This is equivalent to the following:

(i) every compact subset of $D$ is contained in all but finitely many $D_{n}$ 's;

(ii) for every point $z_{0} \in \partial D$, there exists $z_{n} \in \partial D_{n}$ for each $n$ such that $z_{n} \rightarrow z_{0}$.

Remark. A sequence of domains may converge to two different domains. For example, let $D_{n}=\mathbb{C} \backslash((-\infty, n])$. Then $D_{n} \stackrel{\text { Cara }}{\longrightarrow} \mathbb{H}$, and $D_{n} \stackrel{\text { Cara }}{\longrightarrow}-\mathbb{H}$ as well. But two different limit domains of the same domain sequence must be disjoint from each other, because if they have nonempty intersection, then one contains some boundary point of the other, which implies a contradiction.

Lemma A.2. Suppose $D_{n} \stackrel{\text { Cara }}{\longrightarrow} D, f_{n}: D_{n} \stackrel{\text { Conf }}{\rightarrow} E_{n}, n \in \mathbb{N}$, and $f_{n} \stackrel{\text { l.u. }}{\longrightarrow} f$ in $D$. Then either $f$ is constant on $D$, or $f$ is a conformal map on $D$. In the latter case, let $E=f(D)$. Then $E_{n} \stackrel{\text { Cara }}{\longrightarrow} E$ and $f_{n}^{-1} \stackrel{\text { l.u. }}{\longrightarrow} f^{-1}$ in $E$.

Remark. The above lemma resembles the Carathéodory kernel theorem (Theorem 1.8, [11]), but the domains here don't have to be simply connected. The main ingredients in the proof are Rouché's theorem and Koebe's $1 / 4$ theorem. The lemma also holds in the case that $D_{n}$ and $D$ are domains of any Riemann surface, if the metric in the underlying space is used in place of the Euclidean metric for Definition A.1 and locally uniformly convergence. In particular, if we use the spherical metric, then Lemma A.2 holds for domains of $\widehat{\mathbb{C}}$.

\section{B Topology on Interior Hulls}

Let $\mathcal{H}$ denote the set of all interior hulls in $\mathbb{C}$. Recall that for any $H \in \mathcal{H}, \phi_{H}^{-1}$ is defined on $\{|z|>\operatorname{rad}(H)\}$, and for a nondegenerate interior hull, $\psi_{H}(z)=\varphi_{H}^{-1}(z)=\phi_{H}^{-1}(\operatorname{rad}(H) z)$ is defined on $\{|z|>1\}$. It's shown in Section 2.5 of [21 that there is a metric $d_{\mathcal{H}}$ on $\mathcal{H}$ such that for any $H_{n}, H \in \mathcal{H}$, the followings are equivalent:

1. $d_{\mathcal{H}}\left(H_{n}, H\right) \rightarrow 0$

2. $\operatorname{rad}\left(H_{n}\right) \rightarrow \operatorname{rad}(H)$ and $\phi_{H_{n}}^{-1} \stackrel{\text { l.u. }}{\longrightarrow} \phi_{H}^{-1}$ in $\{|z|>\operatorname{rad}(H)\}$.

3. $\mathbb{C} \backslash H_{n} \stackrel{\text { Cara }}{\longrightarrow} \mathbb{C} \backslash H$. 
In particular, we see that rad is a continuous function on $\left(\mathcal{H}, d_{\mathcal{H}}\right)$. Thus, for nondegenerate interior hulls, $d_{\mathcal{H}}\left(H_{n}, H\right) \rightarrow 0$ iff $\psi_{H_{n}} \stackrel{\text { l.u. }}{\longrightarrow} \psi_{H}$ in $\{|z|>1\}$. The following lemma is Lemma 2.2 in 21 .

Lemma B.1. For any $F \in \mathcal{H}$, the set $\{H \in \mathcal{H}: H \subset F\}$ is compact.

Corollary B.2. For any $F \in \mathcal{H}$ and $r>0$, the set $\{H \in \mathcal{H}: H \subset F, \operatorname{rad}(H) \geq r\}$ is compact.

\section{Topology on $\mathbb{H}$-hulls}

From Section 5.2 in [17, there is a metric $d_{\mathcal{H}}$ on the space of $\mathbb{H}$-hulls such that $d_{\mathcal{H}}\left(H_{n} \rightarrow\right.$ $\left.H_{\infty}\right)$ iff $f_{H_{n}} \stackrel{\text { l.u. }}{\longrightarrow} f_{H_{\infty}}$ in $\mathbb{H}$. From Lemma A.2, this implies that $\mathbb{H} \backslash H_{n} \stackrel{\text { Cara }}{\longrightarrow} \mathbb{H} \backslash H_{\infty}$. But $\mathbb{H} \backslash H_{n} \stackrel{\text { Cara }}{\longrightarrow} \mathbb{H} \backslash H_{\infty}$ does not imply $d_{\mathcal{H}}\left(H_{n} \rightarrow H_{\infty}\right)$. A counterexample is $H_{n}=\{z \in \mathbb{H}$ : $|z-2 n| \leq n\}$ and $H_{\infty}=\emptyset$. Since $H_{1} \cdot H_{2}=H_{3}$ iff $f_{H_{1}} \circ f_{H_{2}}=f_{H_{3}}$, the dot product is continuous.

Formula (5.1) in [17] states that for any $\mathbb{H}$-hull $H$, there is a positive measure $\mu_{H}$ supported by $S_{H}^{*}$, the convex hull of $S_{H}$, such that for any $z \in \mathbb{C} \backslash S_{H}^{*}$,

$$
f_{H}(z)=z+\int \frac{-1}{z-x} d \mu_{H}(x) .
$$

In particular, if $H$ is bounded by a crosscut, then $\mu_{H}$ is absolutely continuous w.r.t. the Lebesgue measure, and $d \mu_{H} / d x=\frac{1}{\pi} \operatorname{Im} f_{H}(x)$, where the value of $f_{H}$ on $S_{H}^{*}$ is the continuation of $f_{H}$ from $\mathbb{H}$. If $H$ is approximated by a sequence of $\mathbb{H}$-hulls $\left(H_{n}\right)$, then $\mu_{H}$ is the weak limit of $\left(\mu_{H_{n}}\right)$. We may choose each $H_{n}$ to be bounded by a crosscut, whose height is not bigger than $h+1 / n$, where $h$ is the height of $H$. Then each $\mu_{H_{n}}$ has a density function, whose $L^{\infty}$ norm is not bigger than $(h+1 / n) / \pi$. Thus, $\mu_{H}$ also has a density function, whose $L^{\infty}$ norm is not bigger than $h / \pi$. We use $\rho_{H}$ to denote the density function of $\mu_{H}$. Since $f_{H}: \mathbb{C} \backslash S_{H}^{*} \stackrel{\text { Conf }}{\rightarrow} \mathbb{C} \backslash \widehat{H}^{*}$ and $f_{H}^{\prime}(\infty)=1$, we see that $\operatorname{rad}\left(\widehat{H}^{*}\right)=\operatorname{rad}\left(S_{H}^{*}\right)=\left|S_{H}^{*}\right| / 4$. Thus, $\operatorname{diam}\left(\widehat{H}^{*}\right) \leq 4 \operatorname{rad}\left(\widehat{H}^{*}\right)=\left|S_{H}^{*}\right|$. On the other hand, the diameter of $\widehat{H}^{*}$ is at least twice the height of $H$. So $\left\|\rho_{H}\right\|_{\infty} \leq \frac{\left|S_{H}^{*}\right|}{2 \pi}$.

By approximating any $\mathbb{H}$-hull $H$ using a sequence of $\mathbb{H}$-hulls $\left(H_{n}\right)$, each of which is the union of finitely many mutually disjoint $\mathbb{H}$-hulls bounded by crosscuts in $\mathbb{H}$, we see that $\mu_{H}$ is in fact supported by $S_{H}$. By continuation, C.1 holds for any $z \in \mathbb{C} \backslash S_{H}$. Furthermore, the support of $\mu_{H}$ is exactly $S_{H}$ because from (C.1) $f_{H}$ extends analytically to the complement of the support of $\mu_{H}$, while from Lemma $2.6 f_{H}$ can not be extended analytically beyond $\mathbb{C} \backslash S_{H}$. So we obtain the following lemma.

Lemma C.1. For any $\mathbb{H}$-hull $H, \mu_{H}$ has a density function $\rho_{H}$, whose support is $S_{H}$, and whose $L^{\infty}$ norm is no more than $\frac{\left|S_{H}^{*}\right|}{2 \pi}$. Moreover, C.1) holds for any $z \in \mathbb{C} \backslash S_{H}$.

The following lemma extends Lemma 5.4 in [17], and we now give a proof.

Lemma C.2. For any compact $F \subset \mathbb{R}, \mathcal{H}_{F}:=\left\{H: S_{H} \subset F\right\}$ is compact, and $H_{n} \rightarrow H$ in $\mathcal{H}_{F}$ implies that $f_{H_{n}} \stackrel{\text { l.u. }}{\longrightarrow} f_{H}$ in $\mathbb{C} \backslash F$. 
Proof. Suppose $\left(H_{n}\right)$ is a sequence in $\mathcal{H}_{F}$. Let $\left|F^{*}\right|$ denote the length of the convex hull of $F$. Then for each $n, \rho_{H_{n}}$ is supported by $S_{H_{n}} \subset F$, and the $L^{\infty}$ norm of $\rho_{H_{n}}$ is no more than $\frac{\left|S_{H_{n}}^{*}\right|}{2 \pi} \leq \frac{\left|F^{*}\right|}{2 \pi}$. Thus, $\left(\rho_{H_{n}}\right)$ contains a subsequence $\left(\rho_{H_{n_{k}}}\right)$, which converges in the weak-* topology to a function $\rho$ supported by $F$. From C.1 we see that $f_{H_{n_{k}}}$ converges uniformly on each compact subset of $\mathbb{C} \backslash F$, and if $f$ is the limit function, then $f(z)-z=\int_{F} \frac{-1}{z-x} \rho(x) d x$, $z \in \mathbb{C} \backslash F$. So $f(z)-z \rightarrow 0$ as $z \rightarrow \infty$. This means that $f$ can not be constant. From Lemma A.2 $f$ is a conformal map on $\mathbb{C} \backslash F$. Since $f(z)-z \rightarrow 0$ as $z \rightarrow \infty, \infty$ is a simple pole of $f$. Thus, $f(\mathbb{C} \backslash F)$ contains a neighborhood of $\infty$. Let $G=\mathbb{C} \backslash f(\mathbb{C} \backslash F)$. Then $G$ is compact. Since every $f_{H_{n_{k}}}$ is $\mathbb{R}$-symmetric, so is $f$. Let $H=G \cap \mathbb{H}$. Then $f$ maps $\mathbb{H}$ conformally onto $\mathbb{H} \backslash H$. This implies that $H$ is an $\mathbb{H}$-hull and $f=f_{H}$ on $\mathbb{H}$ because $f(z)-z \rightarrow 0$ as $z \rightarrow \infty$. Since $f$ extends $\left.f_{H}\right|_{\mathbb{H}}$, from Lemma 2.6 we see that $S_{H} \subset F$ and $f=f_{H}$ in $\mathbb{C} \backslash F$. Since $f_{H_{n_{k}}} \stackrel{\text { l.u. }}{\longrightarrow} f_{H}$ in $\mathbb{H}$, we get $H_{n_{k}} \rightarrow H \in \mathcal{H}_{F}$. This shows that $\mathcal{H}_{F}$ is compact. The above argument also gives $f_{H_{n_{k}}} \stackrel{\text { l.u. }}{\longrightarrow} f_{H}$ in $\mathbb{C} \backslash F$. If $H_{n} \rightarrow H$, then any subsequence $\left(H_{n_{k}}\right)$ of $\left(H_{n}\right)$ contains a subsequence $\left(H_{n_{k_{l}}}\right)$ such that $f_{H_{n_{k_{l}}}} \stackrel{\text { l.u. }}{\rightarrow} f_{H}$ in $\mathbb{C} \backslash F$, which implies that $f_{H_{n}} \stackrel{\text { l.u. }}{\longrightarrow} f_{H}$ in $\mathbb{C} \backslash F$.

\section{Topology on $\mathbb{D}$-hulls}

Define a metric $d_{\mathcal{H}}$ on the space of $\mathbb{D}$-hulls such that

$$
d_{\mathcal{H}}\left(H_{1}, H_{2}\right)=\sum_{n=1}^{\infty} \frac{1}{2^{n}} \sup _{|z| \leq 1-1 / n}\left\{\left|f_{H_{1}}(z)-f_{H_{2}}(z)\right|\right\} .
$$

It is clear that $d_{\mathcal{H}}\left(H_{n}, H\right) \rightarrow 0$ iff $f_{H_{n}} \stackrel{\text { l.u. }}{\longrightarrow} f_{H}$ in $\mathbb{D}$. From Lemma A.2, this implies that

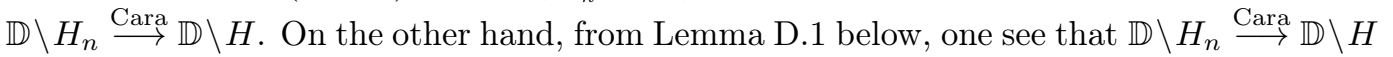
also implies that $H_{n} \rightarrow H$. Since $f_{H_{n}} \stackrel{\text { l.u. }}{\longrightarrow} f_{H}$ in $\mathbb{D}$ implies that $f_{H_{n}}^{\prime}(0) \rightarrow f_{H}^{\prime}(0)$, we see that dcap is a continuous function. Moreover, the dot product is also continuous.

Lemma D.1. For any $M<\infty,\{H: \operatorname{dcap}(H) \leq M\}$ is compact.

Proof. Suppose $\left(H_{n}\right)$ is a sequence of $\mathbb{D}$-hulls with $\operatorname{dcap}\left(H_{n}\right) \leq M$ for each $n$. Then $f_{H_{n}}^{\prime}(0)=e^{-\operatorname{dcap}\left(H_{n}\right)} \geq e^{-M}$. Since $\left(f_{H_{n}}\right)$ is uniformly bounded in $\mathbb{D}$, it contains a subsequence $\left(f_{H_{n_{k}}}\right)$, which converges locally uniformly in $\mathbb{D}$. Let $f$ be the limit. Then $f^{\prime}(0)=\lim _{k \rightarrow \infty} f_{H_{n_{k}}}^{\prime}(0) \geq e^{-M}$. Thus, $f$ is not constant. From Lemma A.2, $f$ is conformal in $\mathbb{D}$. Since $f(0)=\lim _{k \rightarrow \infty} f_{H_{n_{k}}}(0)$ and $f^{\prime}(0)>0$, we see that $f=\left.f_{H}\right|_{\mathbb{D}}$ for some $\mathbb{D}$-hull $H$. Since $f^{\prime}(0) \geq e^{-M}, \operatorname{dcap}(H) \leq M$. From $f_{H_{n_{k}}} \stackrel{\text { l.u. }}{\longrightarrow} f_{H}$ in $\mathbb{D}$ we get $H_{n_{k}} \rightarrow H$.

Remark. We may compactify the space of $\mathbb{D}$-hulls by adding one element $H_{\infty}$ with the associated function $f_{H_{\infty}} \equiv 0$ in $\mathbb{D}$, and defining the metric $d_{\mathcal{H}}$ in the extended space using (D.1).

Lemma D.2. For any compact $F \varsubsetneqq \mathbb{T}, \mathcal{H}_{F}:=\left\{H: S_{H} \subset F\right\}$ is compact.

Proof. Let $H \in \mathcal{H}_{F}$. From conformal invariance, the harmonic measure of $\mathbb{T} \backslash \widehat{H}$ in $\mathbb{D} \backslash H$ seen from 0 equals to the harmonic measure of $\mathbb{T} \backslash S_{H}$ in $\mathbb{D}$ seen from 0 , which is bounded 
below by $|\mathbb{T} \backslash F| /|\mathbb{T}|>0$. This implies that the distance between 0 and $H$ is bounded below by a positive constant $r$ depending on $F$, which then implies that dcap $(H)$ is bounded above by $-\ln (r)<\infty$. From Lemma D.1, we see that $\mathcal{H}_{F}$ is relatively compact.

It remains to show that $\mathcal{H}_{F}$ is bounded. Let $\left(H_{n}\right)$ be a sequence in $\mathcal{H}_{F}$, which converges to $H$. We need to show that $H \in \mathcal{H}_{F}$. Since $H_{n} \in \mathcal{H}_{F}$, each $f_{H_{n}}$ is analytic in $\widehat{\mathbb{C}} \backslash F$. We

have $f_{H_{n}} \stackrel{\text { l.u. }}{\longrightarrow} f_{H}$ in $\mathbb{D}$. From $\mathbb{T}$-symmetry, $f_{H_{n}} \stackrel{\text { l.u. }}{\longrightarrow} f_{H}$ in $\mathbb{D}^{*}$. Let $J=\{|z|=2\} \subset \mathbb{D}^{*}$. Then $f_{H_{n_{k}}} \rightarrow f_{H}$ uniformly on $J$. Sine $f_{H_{n}}$ maps $\{|z|<2\} \backslash F$ into the Jordan domain bounded by $f_{H_{n}}(J)$, we see that the family $\left(f_{H_{n}}\right)$ is uniformly bounded in $\{|z|<2\} \backslash F$. So it contains a subsequence $\left(f_{H_{n}}\right)$, which converges locally uniformly in $\{|z|<2\} \backslash F$. The limit function is analytic in $\{|z|<2\} \backslash F$ and agrees with $f_{H}$ on $\mathbb{D}$, which implies that $f_{H}$ extends analytically across $\mathbb{T} \backslash F$. So $S_{H} \subset F$, i.e., $H \in \mathcal{H}_{F}$.

There is an integral formula for $\mathbb{D}$-hulls which is similar to (C.1). For any $\mathbb{D}$-hull $H$, there is a positive measure $\mu_{H}$ with support $S_{H}$ such that

$$
f(z)=z \cdot \exp \left(\int_{\mathbb{T}}-\frac{x+z}{x-z} d \mu_{H}(x)\right), \quad z \in \mathbb{C} \backslash S_{H},
$$

and $H_{n} \rightarrow H$ iff $\mu_{H_{n}} \rightarrow \mu_{H}$ weakly. Moreover, $\mu_{H}$ is absolutely continuous w.r.t. the Lebesgue measure on $\mathbb{T}$, and the density function is bounded. From this integral formula, it is easy to get the following lemma.

Lemma D.3. For any compact $F \subset \mathbb{T}, H_{n} \rightarrow H$ in $\mathcal{H}_{F}$ implies that $f_{H_{n}} \stackrel{\text { l.u. }}{\longrightarrow} f_{H}$ in $\mathbb{C} \backslash F$.

\section{References}

[1] Lars V. Ahlfors. Conformal invariants: topics in geometric function theory. McGrawHill Book Co., New York, 1973.

[2] Kari Astala, Peter Jones, Antti Kupiainen, and Eero Saksman. Random Conformal Weldings. Acta. Math., 207(2):203-254, 2011.

[3] Christopher Bishop, Conformal welding and Koebe's theorem, Annals of Mathematics, 166: 613-656, 2007

[4] Julien Dubédat. Commutation relations for SLE, Communications on Pure and Applied Mathematics, 60(12):1792-1847, 2007.

[5] Peter W. Jones and Stanislav K. Smirnov. Removability theorems for Sobolev functions and quasiconformal maps. Ark. Mat., 38(2):263-279, 2000.

[6] Gregory F. Lawler. Conformally Invariant Processes in the Plane. Am. Math. Soc., Providence, RI, 2005.

[7] Gregory F. Lawler, Oded Schramm and Wendelin Werner. Values of Brownian intersection exponents I: Half-plane exponents. Acta Math., 187(2):237-273, 2001.

[8] Olli Lehto and Kalle Virtanen, Quasiconformal mappings in the plane, Springer-Verlag, 1973.

[9] Jason Miller and Scott Sheffield, Imaginary geometry III, arXiv:1201.1498, 2012. 
[10] Christian Pommerenke. On the Löwner differential equation. Mich. Math. J. 13:435443, 1968.

[11] Christian Pommerenke. Boundary behaviour of conformal maps. Springer-Verlag, Berlin Heidelberg New York, 1991.

[12] Daniel Revuz and Marc Yor. Continuous Martingales and Brownian Motion. Springer, Berlin, 1991.

[13] Steffen Rohde and Oded Schramm. Basic properties of SLE. Ann. Math., 161(2):883924, 2005.

[14] Oded Schramm. Scaling limits of loop-erased random walks and uniform spanning trees. Israel J. Math., 118:221-288, 2000.

[15] Oded Schramm and David B. Wilson. SLE coordinate changes. New York Journal of Mathematics, 11:659-669, 2005.

[16] Scott Sheffield. Conformal weldings of random surfaces: SLE and the quantum gravity zipper. In preprint, arXiv:1012.4797v1.

[17] Dapeng Zhan. The Scaling Limits of Planar LERW in Finitely Connected Domains. Ann. Probab., 36(2):467-529, 2008.

[18] Dapeng Zhan. Reversibility of chordal SLE. Ann. Probab., 36(4):1472-1494, 2008.

[19] Dapeng Zhan. Duality of chordal SLE. Invent. Math., 174(2):309-353, 2008.

[20] Dapeng Zhan. Duality of chordal SLE, II. Ann. I. H. Poincare-Pr., 46(3):740-759, 2010.

[21] Dapeng Zhan. Continuous LERW started from interior points. Stoch. Proc. Appl., 120:1267-1316, 2010.

[22] Dapeng Zhan. Reversibility of whole-plane SLE. In preprint, arXiv:1004.1865.

[23] Dapeng Zhan. Ergodicity of the tip of an SLE curve. In preprint, arXiv:1310.2573. 